Part of Journal of Research of the National Bureau of Standards, Volume 14, February 1935

\title{
IMPACT AND STATIC TENSILE PROPERTIES OF BOLTS
}

\author{
By Herbert L. Whittemore, George W. Nusbaum, and Edgar O. Seaquist
}

\section{ABSTRACT}

This investigation was made to determine the properties of bolts under impact tensile loading and also under static tensile loading. 360 specimens were tested, representing all possible combinations of five different materials (chromium-nickel steel, cold-rolled steel, monel metal, bronze, and brass), four different bolt diameters $(3 / 8,1 / 2,5 / 8,3 / 4$ in.), and three different forms of screw threads (American National coarse, American National fine, and Dardelet). These threads are often used by engineers in this country for bolts. The U. S. Standard threads are almost the same as the American National coarse threads and the SAE threads almost the same as the American National fine threads. The bolts of different diameters were geometrically similar, the length between the head and the bearing face of the nut being five times the diameter, the thread extending inward from the face of the nut one diameter. In all cases the impact work for bolts with American National coarse threads was less than for bolts of the same size and material with American National fine threads. Except for the brass bolts and those cold-rolled steel bolts which showed brittle failures, the impact work for bolts with American National fine threads was approximately the same as for bolts of the same size and material with Dardelet threads. In all cases the impact work for bolts with Dardelet threads was much greater than for bolts of the same size and material having American National coarse threads.

Similar relations were observed for the static work and the maximum static load.

For bolts of the same size and having the same threads the bolt efficiencies were approximately the same for all of the materials.

\section{CONTENTS}

I. Introduction

II. Description of screw threads

III. Material _... 144

1. Kinds of materials

2. Identification

3. Vickers numbers.........

4. Chemical compositions _.

5. Mechanical properties

IV. Specimens

1. Manufacture

2. Measurement of specimens

V. Method of testing

1. General

2. Impact tests

3. Static tests

VI. Results

VII. Discussion

1. Impact tests . .

2. Static tests

3. Comparison of impact and static properties....... 180

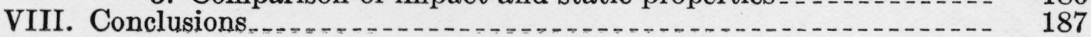




\section{INTRODUCTION}

Bolts are used in machines and structures to fasten together parts which cannot readily be made of one piece, or parts which may be removed for adjustment, cleaning, or repairs. In many cases these fastenings are subjected in service to static and to impact tensile loads. The designing engineer should know not only the greatest load which a bolt will sustain without failing but also the work (force times distance) required to rupture the bolt if, under unusual circumstances, the bolted structure should be subjected either to steady or to impact loads greater than the working loads for which the structure was designed.

The resistance to static loads depends upon the tensile strength of the bolt with nut. The resistance to impact loads, however, depends upon the work required to rupture the bolt under suddenly applied loads. This "impact work" depends upon the load-stretch curve under impact. This will be different from the load-stretch curve under static conditions and would be affected differently by the shape and surface of the threads and the character of the material.

Many investigations have shown that for metals under either tensile or transverse loading, the work required to rupture the specimen under loads applied slowly (the "static work") is not equal to the work required to rupture it under loads applied suddenly (the impact work). Similar differences between the static work and the impact work are to be expected for bolts.

A few investigators have studied the static and, sometimes, the impact strength of bolts. Their results were used as a guide when preparing the test program for this investigation. The effect of decreasing the cross-sectional area of the shank of the bolt to about the cross-sectional area at the bottom of the threads has been discussed by Kimball and Barr. ${ }^{1}$ They point out that although decreasing the cross-sectional area lowers somewhat both the torsional and the tensile strength and the stiffness, it increases the impact work many times because it increases the stretch. They discuss decreasing the cross-sectional area by reducing the diameter of the shank, by longitudinal flutes and, preferably, by an axial hole. Drop tests upon bolts for Prof. Sweet's straight line engine showed that the impact work was increased about nine times by an axial hole.

The effect of differences in the pitch of the thread was studied by Major William R. King, U. S. Engineers. ${ }^{2}$ His static tensile tests made at Watertown Arsenal on wrought-iron bolts, 11/2 in. diameter having $\vee$ threads, showed that the static work for bolts having 18 threads per inch was four times that for similar bolts having 6 threads per inch.

The shape of thread was considered by Langenberg. ${ }^{3}$ His tensile impact tests at Watertown Arsenal on specimens having Acme threads (flat bottoms) showed greater stretch and greater impact work than similar specimens having a semicircular groove at the bottom of the thread.

\footnotetext{
1 Elements of Machine Design, p. 178 (1913).

2 Experiments with bolts and screw threads. Trans. Am. Inst. Mining Engrs. 14,90 (June 1885 and May 1886).

3 Investigation of failure of elevating screws on $14 \mathrm{in} . \quad$ D. C. no.13 and $14 \mathrm{in} . \quad$ D. C. no.14, Tests of Metals p. 44 (1917). Experimental data obtained on the Charpy impact machine. Tests of Metals, p. 222 (1918). Also this latter paper by F. C. Langenberg. Bul. Am. Inst. Mining and Met. Engrs. no. 152, p. 1471 (Aug. 1919).
} 
Beyer ${ }^{4}$ made static tensile and tensile impact tests on cold-drawn steel bolts having Dardelet and U. S. Standard threads. He reported that bolts having Dardelet threads gave greater static tensile strength, greater stretch, and greater resistance to impact than similar bolts having U. S. Standard threads.

He found further ${ }^{5}$ that the static tensile strength and the static work depended upon the length of the thread exposed between the head of the bolt and the face of the nut. As the length of exposed thread was decreased, the stretch under static load and also the static work decreased markedly being only one-half to one-third as great when no threads were exposed as when the exposure was two or three times the diameter of the bolt.

To keep this investigation within practicable limits it was decided to study the effect on the static work and impact work of differences in only three variables. They were the shape of the thread, the material, and the size of the bolt. The three shapes of thread which are used for most commercial bolts in this country were chosen. They were the American National coarse thread, the American National fine thread, and the Dardelet thread. The differences between the American National coarse thread and the U. S. Standard thread, and between the American National fine thread and the SAE thread are so small that there is no reason to believe that there would be appreciable differences in the static or the impact works. Therefore, the U. S. Standard thread and the SAE thread were not included. The results showed the effect of differences in the pitch for threads having the same profile (shape) as studied by King and the effect of differences in the profile of commercial threads as studied by Langenberg and Beyer.

The materials, chromium-nickel steel, cold-rolled steel, monel metal, bronze, and brass, were chosen as representative of the kinds of material which are much used for commercial bolts and nuts. It is believed that from the results on these five materials, the static work and the impact work for other materials can be estimated with sufficient accuracy for engineering purposes. If all the different materials used for bolts and nuts had been included the cost of this investigation would have been prohibitive.

Through the courtesy and cooperation of the Ordnance Department of the U. S. Army the impact tests were made in the large Charpy machine at Watertown Arsenal. This machine ${ }^{6}$ having a nominal capacity of $2,170 \mathrm{ft}-\mathrm{lb}(300 \mathrm{~kg}-\mathrm{m})$ is the largest impact machine of the Charpy type in this country. Nisley gives the weight of the pendulum as $212.46 \mathrm{lb}$, the radius to the center of gravity as $5.34 \mathrm{ft}$, the maximum starting angle as $160^{\circ}$, the free return angle corresponding to the maximum starting angle as $158^{\circ}$, the velocity of impact (maximum starting angle), as $28.65 \mathrm{fps}$, the (actual) capacity as $2,203.2 \mathrm{ft}-\mathrm{lb}$, the distance from the center of the specimen to the axis of rotation as $6.56 \mathrm{ft}$, the period of oscillation as $2.77 \mathrm{sec}-$ onds, and the weight of the block or tup as $5.07 \mathrm{lb}$.

\footnotetext{
4 Reports no. 2162 (Nov. 1929) and no. 2162A (Feb. 1930), Comparative Shock Resistance of Standard V Thread and Nut Connections and Dardelet Thread and Nut Connections. Columbia University (New York, N. Y.).

S Report no. 2207 (June 1930), Effect of Length of Thread Exposure upon the Static Tensile Strength and Energy to Rupture of Standard V and Dardelet Thread and Nut Connections. Columbia University (New York, N. Y.).

${ }^{6}$ Harold $\mathrm{A}$. Nisley, The relation between the dynamic and the static tensile tests. Army Ordnance 4, no. 20, 88-93 (Sept.-Oct. 1923).
} 
For some of the materials it was estimated that specimens of the desired shape having a diameter greater than $3 / 4$ in. might not be ruptured in this impact machine. It was estimated, therefore, that in this machine bolts made from the strongest and most ductile material and having a diameter of $3 / 4$ in. could be tested and that the results obtained on bolts made from the weakest and least ductile material and having a diameter of $3 / 8$ in. would be sufficiently accurate.

As bolts having a diameter less than $3 / 8$ in. are seldom used under severe service conditions, the sizes chosen for the specimens were $3 / 8$, $1 / 2,5 / 8$, and $3 / 4$ in. diameter.

To avoid the differences in work found by Beyer ${ }^{7}$ caused by differences in the length of thread exposed between the head of the bolt

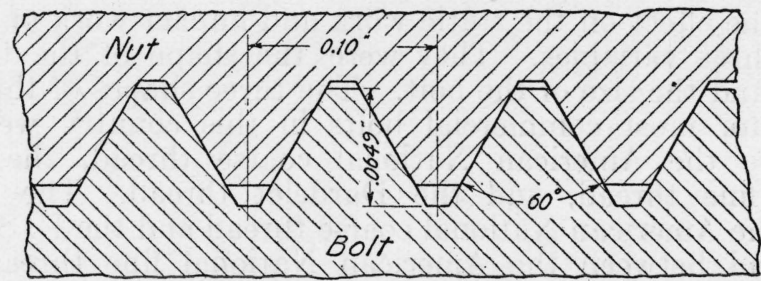

Figure 1.-Section of the American National coarse thread for a bolt having a diameter of $3 / 4$ inch.

and the face of the nut, all the tests were made with the thread exposure equal to one diameter of the bolt. It is believed that this is about the average thread exposure for commercial bolts under service conditions.

\section{DESCRIPTION OF SCREW THREADS}

The dimensions of the American National thread (coarse and fine) nuts and bolts are given in the Report of the National Screw Thread

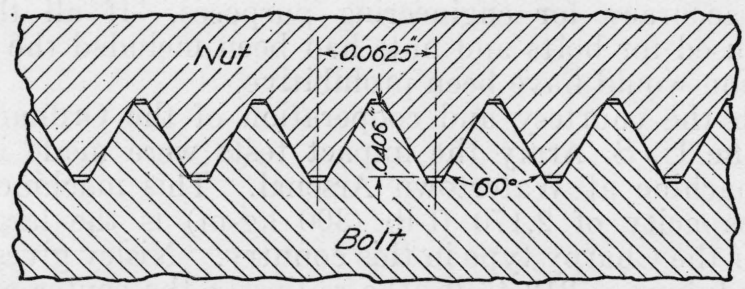

Figure 3.-Section of the American National fine thread for a bolt having a diameter of $3 / 4$ inch.

Commission, Misc. Pub. BS M89 (revised 1928). Those of the U. S. Standard thread are given in the Report of the Special Committee on a Uniform System of Screw Threads, Journal of the Franklin Institute, volume 49, pages 53 to 57 (1865) (adopted March 16, 1865 , l. c. page 280 ).

The dimensional differences between the American National coarse thread and the U. S. Standard thread are so small that the bolts and nuts are usually interchangeable.

\footnotetext{
7 Report no. 2207 (June 1930), Effect of Length of Thread Exposure upon the Static Tensile Strength and Energy to Rupture of Standard V and Dardelet Thread and Nut Connections. Columbia University, (New York, N. Y.).
} 


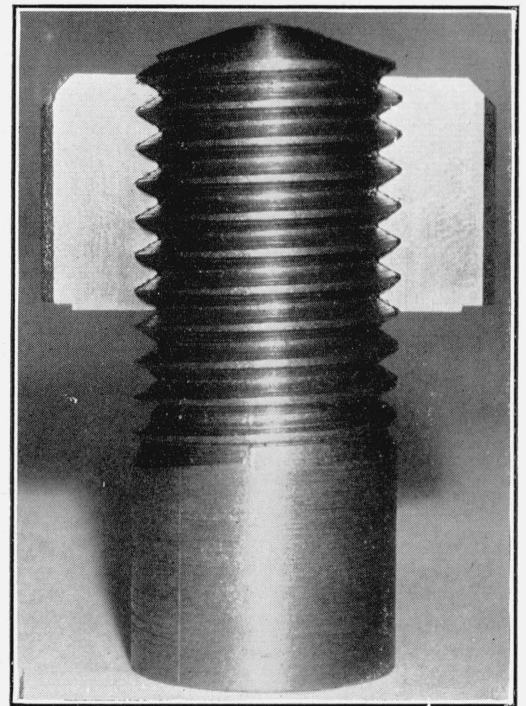

Figure 2.-American National coarse thread, diameter of bolt $3 / 4$ inch.

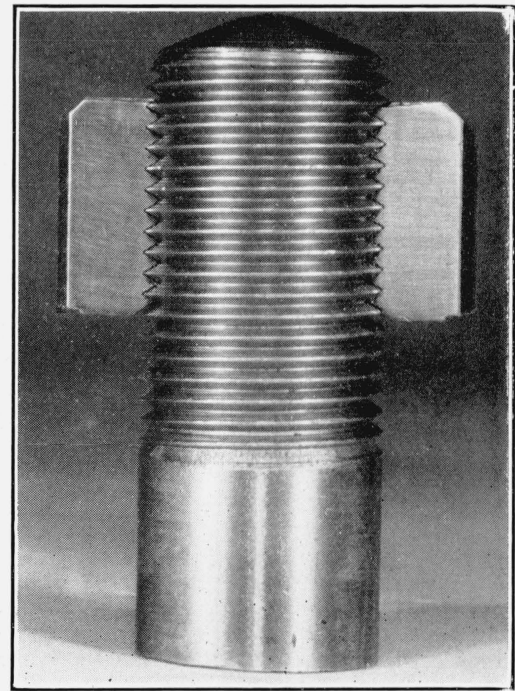

Figure 4.-American National fine thread, diameter of bolt $3 / 4$ inch. 


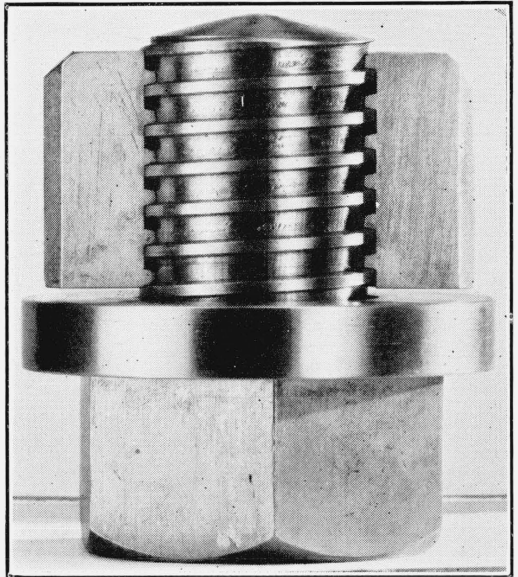

Figure 7.-The Dardelet thread, diameter of bolt $3 / 4$ inch. Position of the nut when it rotates freely on the bolt.

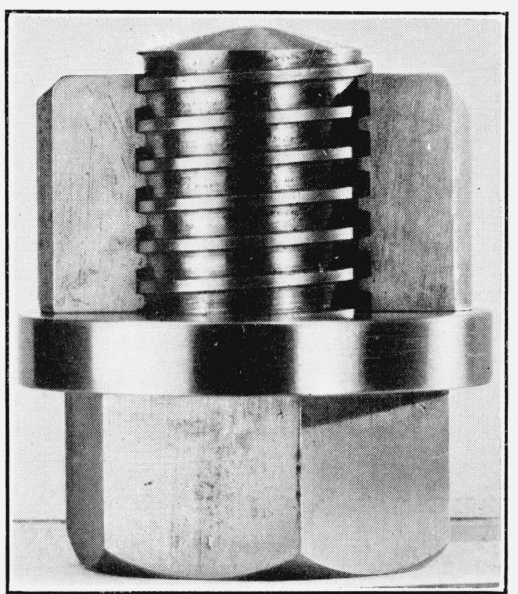

FiguRE 8.-Dardelet thread, diameter of bolt $3 / 4$ inch.

Position of the nut when thrust faces are in contact and the helical cones are elastically compressed, resisting unscrewing of the nut. 
A drawing of the American National coarse thread for a $3 / 4 \mathrm{in}$. bolt is shown in figure 1 and a photograph in figure 2 . There are 10 threads per inch on this bolt. A drawing of the American National fine thread is shown in figure 3 for a $3 / 4$ in. bolt and a photograph in figure 4. There are 16 threads per inch on this bolt. For all diameters of bolt the pitch of the American National fine thread is less than that of the American National coarse thread.

Drawings of the Dardelet thread for a $3 / 4$ in. bolt are shown in figures 5 and 6 , and photographs in figures 7 and 8 . The inclination

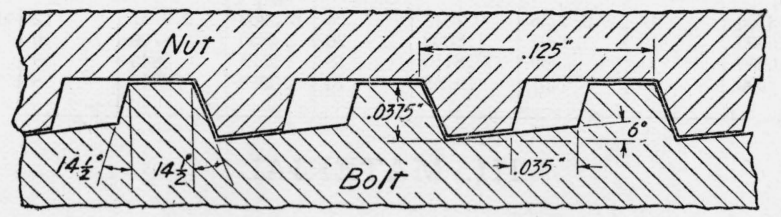

FIgURE 5.-Section of the Dardelet thread for a bolt having a diameter of $3 / 4$ inch. Position of the nut when it rotates freely on the bolt.

of the sides or thrust faces of the threads is the same as that of the Acme thread, i. e., $14 \frac{12^{\circ}}{}$ to the normal to the axis of the bolt. The surfaces at the major diameters are cylindrical with clearance between the nut and the bolt. The surfaces at the minor diameter of both the bolt and the nut are tapered, forming oblique helicoids with a generating angle of $6^{\circ}$ to the axis of the bolt. For convenience these surfaces will, hereafter, be called "helical cones." When the nut is screwed on the bolt there is clearance between the threads, as shown in figures 5 and 7 , and the nut rotates freely. When the nut

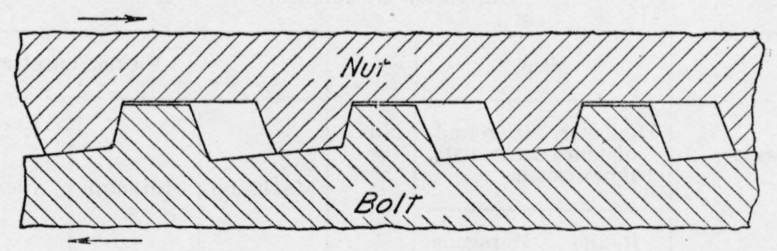

Figure 6.-Section of the Dardelet thread for a bolt having a diameter of $3 / 4$ inch. Position of the nut when the thrust faces are in contact and the helical cones are elastically compressed resisting unscrewing of the nut.

is screwed down into contact with the parts to be clamped, the helical cones come into contact, and as the nut is tightened they are compressed elastically until the thrust faces of the threads come into contact as shown in figures 6 and 8 . When the bolt is under the working tension most of the load is carried by the thrust faces. The elastic compression of the helical cones increases the frictional resistance of the nut to rotation.

The nominal dimensions of the threads and the number of threads for each size of specimen are given in table 1 . 
TABLE 1.-Nominal dimensions of threads and number of threads

\begin{tabular}{|c|c|c|c|c|c|c|c|c|c|c|}
\hline \multirow{2}{*}{$\begin{array}{c}\text { Nominal } \\
\text { size of } \\
\text { speci- } \\
\text { men }\end{array}$} & \multirow{2}{*}{$\begin{array}{c}\text { Basic } \\
\text { major } \\
\text { diameter } \\
\text { for all } \\
\text { threads }\end{array}$} & \multicolumn{3}{|c|}{ American $\begin{array}{c}\text { National coarse } \\
\text { thread }\end{array}$} & \multicolumn{3}{|c|}{$\begin{array}{c}\text { American National fine } \\
\text { thread }\end{array}$} & \multicolumn{3}{|c|}{ Dardelet thread } \\
\hline & & $\begin{array}{l}\text { Number } \\
\text { of } \\
\text { threads } \\
\text { per inch }\end{array}$ & $\begin{array}{c}\text { Basic } \\
\text { minor } \\
\text { diameter }\end{array}$ & $\begin{array}{c}\text { Ratio of } \\
\text { diam- } \\
\text { eters- } \\
\text { minor: } \\
\text { major }\end{array}$ & $\begin{array}{c}\text { Number } \\
\text { of } \\
\text { threads } \\
\text { per inch }\end{array}$ & $\begin{array}{c}\text { Basic } \\
\text { minor } \\
\text { diam- } \\
\text { eter }\end{array}$ & $\begin{array}{c}\text { Ratio of } \\
\text { diam- } \\
\text { eters- } \\
\text { minor: } \\
\text { major }\end{array}$ & $\begin{array}{l}\text { Number } \\
\text { of } \\
\text { threads } \\
\text { per inch }\end{array}$ & $\begin{array}{c}\text { Basic } \\
\text { minor } \\
\text { diam- } \\
\text { eter }\end{array}$ & $\begin{array}{c}\text { Ratio } \\
\text { of diam- } \\
\text { eters- } \\
\text { minor: } \\
\text { major }\end{array}$ \\
\hline $\begin{array}{l}\text { in. } \\
3 / 8 \\
1 / 2 \\
5 / 8 \\
3 / 4\end{array}$ & $\begin{array}{l}\text { in. } \\
0.3750 \\
.5000 \\
.6250 \\
.7500\end{array}$ & $\begin{array}{l}16 \\
13 \\
11 \\
10\end{array}$ & $\begin{array}{l}\text { in. } \\
0.2938 \\
.4001 \\
.5069 \\
.6201\end{array}$ & $\begin{array}{l}0.7835 \\
.8002 \\
.8110 \\
.8268\end{array}$ & $\begin{array}{l}24 \\
20 \\
18 \\
16\end{array}$ & $\begin{array}{c}\text { in. } \\
0.3209 \\
.4350 \\
.5528 \\
.6688\end{array}$ & $\begin{array}{l}0.8557 \\
.8700 \\
.8845 \\
.8917\end{array}$ & $\begin{array}{r}12 \\
10 \\
8 \\
8\end{array}$ & $\begin{array}{c}\text { in. } \\
0.3250 \\
.4400 \\
.5500 \\
.6750\end{array}$ & $\begin{array}{l}0.8667 \\
.8800 \\
.8800 \\
.9000\end{array}$ \\
\hline
\end{tabular}

\section{MATERIAL}

\section{KINDS OF MATERIALS}

The materials for the specimens were chromium-nickel steel (heat treated), cold-rolled steel, monel metal, bronze, and brass.

All nuts were made from hexagonal bars except those of chromiumnickel steel, which were made from round bars because hexagonal bars were unobtainable from stock. The diameter of these round bars was equal to the distance across flats of hexagonal nuts of the same nominal size. As the nuts were only hand tight when assembled for the tests no flats were required. The sizes of the bars are given in table 2 .

TABLE 2.-Size and Vickers numbers of material

Chromium-Nickel Steel

\begin{tabular}{|c|c|c|c|c|c|c|c|}
\hline \multirow{3}{*}{ Purpose } & \multicolumn{2}{|c|}{ Size } & \multirow{3}{*}{$\begin{array}{l}\text { Num- } \\
\text { ber of } \\
\text { bars }\end{array}$} & \multicolumn{4}{|c|}{ Vickers number } \\
\hline & \multicolumn{2}{|c|}{$\begin{array}{l}\text { Diameter, if round; } \\
\text { distance across flats, } \\
\text { if hexagonal }\end{array}$} & & \multirow{2}{*}{ Maximum } & \multirow{2}{*}{ Minimum } & \multirow{2}{*}{ Average } & \multirow[t]{2}{*}{ Load } \\
\hline & Round & Hexagonal & & & & & \\
\hline $\begin{array}{l}\text { Bolts. } \\
\text { Nuts. - }\end{array}$ & $\begin{array}{c}\text { in. } 11 / 8 \\
13 \\
11 / 8\end{array}$ & $\begin{array}{c}\text { in. } \\
\end{array}$ & $\begin{array}{l}4 \\
1 \\
1 \\
1 \\
1\end{array}$ & $\begin{array}{l}308 \\
291 \\
307 \\
291 \\
300\end{array}$ & $\begin{array}{l}265 \\
289 \\
298 \\
287 \\
294\end{array}$ & $\begin{array}{l}279 \\
290 \\
302 \\
289 \\
297\end{array}$ & $\begin{array}{ll}\mathrm{kg} & \\
50 \\
50 \\
50 \\
50 \\
50\end{array}$ \\
\hline
\end{tabular}

Cold-Rolled Steel

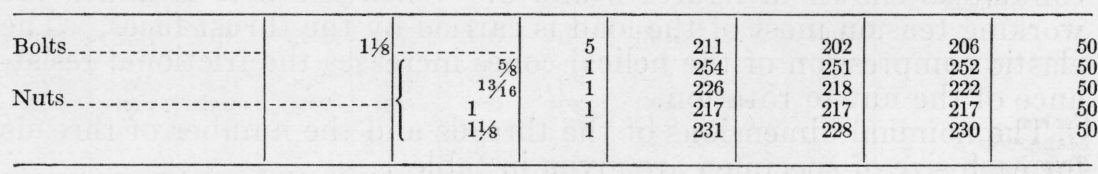

Monel Metal

\begin{tabular}{|c|c|c|c|c|c|c|c|}
\hline $\begin{array}{l}\text { Bolts. } \\
\text { Nuts.. }\end{array}$ & $11 / 8$ & $\begin{array}{r}5 / 8 \\
13 / 16 \\
11 / 8\end{array}$ & $\begin{array}{l}4 \\
1 \\
1 \\
1 \\
1\end{array}$ & $\begin{array}{l}277 \\
202 \\
202 \\
204 \\
204\end{array}$ & $\begin{array}{l}254 \\
199 \\
199 \\
203 \\
189\end{array}$ & $\begin{array}{l}262 \\
200 \\
200 \\
204 \\
196\end{array}$ & $\begin{array}{l}50 \\
50 \\
50 \\
50 \\
50\end{array}$ \\
\hline
\end{tabular}


TABLE 2.-Size and Vickers numbers of material-Continued

Bronze

\begin{tabular}{|c|c|c|c|c|c|c|c|}
\hline \multirow{3}{*}{ Purpose } & \multicolumn{2}{|c|}{ Size } & \multirow{3}{*}{$\begin{array}{l}\text { Num- } \\
\text { ber of } \\
\text { bars }\end{array}$} & \multicolumn{4}{|c|}{ Vickers number } \\
\hline & \multicolumn{2}{|c|}{$\begin{array}{l}\text { Diameter, if round; } \\
\text { distance across flats, } \\
\text { if hexagonal }\end{array}$} & & \multirow[t]{2}{*}{ Maximum } & \multirow[t]{2}{*}{ Minimum } & \multirow[t]{2}{*}{ Average } & \multirow[t]{2}{*}{ Load } \\
\hline & Round & Hexagonal & & & & & \\
\hline $\begin{array}{l}\text { Bolts. } \\
\text { Nuts. }\end{array}$ & in. & $\begin{array}{l}\text { in. } \\
13 / 86 \\
116 \\
11 / 8\end{array}$ & $\begin{array}{l}5 \\
1 \\
1 \\
1 \\
1\end{array}$ & $\begin{array}{l}227 \\
183 \\
202 \\
212 \\
201\end{array}$ & $\begin{array}{l}193 \\
179 \\
202 \\
209 \\
190\end{array}$ & $\begin{array}{l}214 \\
181 \\
202 \\
210 \\
196\end{array}$ & $\begin{aligned} & \text { kg. } \\
& 50 \\
& 50 \\
& 50 \\
& 50 \\
& 50\end{aligned}$ \\
\hline
\end{tabular}

Brass

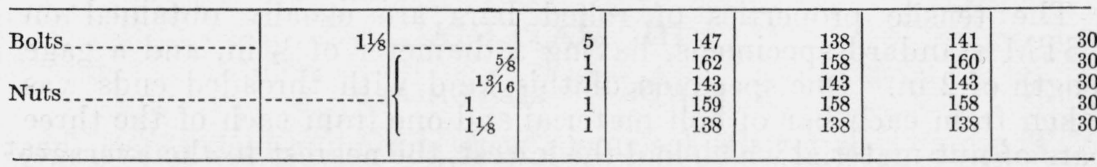

\section{IDENTIFICATION}

An impression was made with a knurl on the surface of each bar for its entire length and the number of the bar stamped upon it every inch. As a portion of the original surface of the bar remained on each finished bolt and nut, the material was identified by the pattern of the knurling and the bar by the number.

\section{VICKERS NUMBERS}

Pieces $3 / 8$ in. long were cut from both ends of each bar and the cut surface machined and polished. Using a Vickers machine and a diamond pyramid, three identations were made on the polished surface of each piece and the Vickers numbers determined. A load of $30 \mathrm{~kg}$ was used for brass and a load of $50 \mathrm{~kg}$ for all the other materials. The Vickers numbers are given in table 2 .

\section{CHEMICAL COMPOSITIONS}

The results of chemical analyses of the material are given in table 3 . The samples were taken from the bars having the nearest to the average Vickers number for the bolt stock and for the nut stock of each material.

TABLE 3.-Chemical analyses of material

\begin{tabular}{|c|c|c|c|c|c|c|c|c|c|c|}
\hline \multirow{2}{*}{ Element } & \multicolumn{2}{|c|}{$\begin{array}{l}\text { Chromium- } \\
\text { nickel steel }\end{array}$} & \multicolumn{2}{|c|}{$\begin{array}{l}\text { Cold-rolled } \\
\text { steel }\end{array}$} & \multicolumn{2}{|c|}{ Monel metal } & \multicolumn{2}{|c|}{ Bronze } & \multicolumn{2}{|c|}{ Brass } \\
\hline & Bolt & Nut & Bolt & Nut & Bolt & Nut & Bolt & Nut & Bolt & Nut \\
\hline Carbon & $\begin{array}{l}\text { Per- } \\
\text { cent } \\
0.37\end{array}$ & $\begin{array}{c}\text { Per- } \\
\text { cent } \\
0.33\end{array}$ & $\begin{array}{l}\text { Per- } \\
\text { cent } \\
0.12\end{array}$ & $\begin{array}{l}\text { Per- } \\
\text { cent } \\
0.18\end{array}$ & $\begin{array}{l}\text { Per- } \\
\text { cent }\end{array}$ & $\begin{array}{l}\text { Per- } \\
\text { cent }\end{array}$ & $\begin{array}{l}\text { Per- } \\
\text { cent }\end{array}$ & $\begin{array}{l}\text { Per- } \\
\text { cent }\end{array}$ & $\begin{array}{l}\text { Per- } \\
\text { cent }\end{array}$ & $\begin{array}{l}\text { Per- } \\
\text { cent }\end{array}$ \\
\hline Manganese.. & .55 & .57 & .84 & .92 & 1.3 & 1.2 & 0.9 & 1.2 & & \\
\hline Phosphorus. & 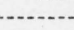 & $\cdots$ & .099 & .115 & & & $\cdots$ & $\cdots$ & & \\
\hline $\begin{array}{l}\text { Sulphur. } \\
\text { Silicon. }\end{array}$ & $\cdots+x+y$ & -- & .12 & .12 & & & 20 & 3 & & \\
\hline Chromium. & .52 & .63 & & & & & 2.0 & & & \\
\hline Nickel....... & 1. 28 & 1. 24 & & & 65.9 & 67.2 & & 1 & & 0.02 \\
\hline Copper-.. & - & - n & & & 31.2 & 29.8 & 95.9 & 95.1 & 61.7 & 62.4 \\
\hline Zinc 1 & - nn- & -n. & & $\ldots$ & & & .15 & .39 & 34.89 & 34.36 \\
\hline Iron & .... & $-\ldots$ & & .. & 1.7 & 1.8 & .15 & .21 & .01 & .02 \\
\hline Tin... & 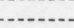 & & & & & & & -... & .1 & .1 \\
\hline
\end{tabular}

${ }_{6}^{1}$ By difference. 
The chromium-nickel steel was similar to SAE 3140 and had been heat treated to comply with ASTM A96-27, class C. The manufacturer stated that each lot was tested and was guaranteed to comply with this specification. The cold-rolled steel was described by the manufacturer as "cold finished screw stock" similar to SAE 1112. It was also similar to the Bessemer screw-steel grade in ASTM A108-30.

The bronze was a copper-silicon-manganese alloy similar to FS QQ-C-591. For convenience it is called "bronze" in this paper.

The brass was a free-cutting brass similar to grade B of FS QQ-C611 and ASTM B16-29. For convenience it is called "brass" in this paper.

\section{MECHANICAL PROPERTIES}

The tensile properties of rolled bars are usually obtained on ASTM standard specimens, having a diameter of $1 / 2$ in. and a gage length of $2 \mathrm{in}$. One specimen of this kind with threaded ends was taken from each bar of bolt material and one from each of the three bars of nut material which had the lowest, the nearest to the average, and the highest Vickers numbers. There was not sufficient nut material to take a specimen from each bar. The ultimate tensile strength, elongation, and reduction of area were determined.

Additional specimens were taken to determine accurately the proportional limit and the Young's modulus of elasticity. From each bolt and nut material one additional specimen was taken from the bar having the nearest to the average Vickers number and being, therefore, probably the most representative. For the bolt materials the additional specimen had a diameter of $1 / 2$ in. and a gage length of 8 in. The ends were threaded. A Ewing extensometer (gage length 8 in.) was used on these specimens. As there was insufficient nut material for additional specimens having an 8-in. gage length, the additional specimens for the nut material were ASTM standard specimens of $1 / 2$ in. diameter and 2 in. gage length.

Two Tuckerman optical strain gages ${ }^{8}$ (gage length 2 in.), one on each side, were used on these specimens.

The proportional limit and the Young's modulus of elasticity for the additional specimens were determined from stress-strain diagrams. The proportional limit reported was the stress at which the stressstrain curve deviated from a straight line by a strain of $0.00001 \mathrm{in}$./in.

All the specimens were tested in a screw-power, beam and poise testing machine having a capacity of $50,000 \mathrm{lb}$. Calibration of this machine showed that the errors in the indicated load did not exceed 1 percent.

The mechanical properties of the materials are given in table 4 .

8 Proc. Am. Soc. Testing Materials, 23, part 2, 602-610 (1923). Engineering (London) 116, 222-223 (1923). World Eng. Congr. (Tokyo, Japan) Paper 335, p. 33-36. 
TABLE 4.-Mechanical properties of material

Chromium-Nickel Steel

\begin{tabular}{|c|c|c|c|c|c|c|c|c|c|c|c|c|c|}
\hline Purpose & 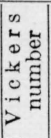 & 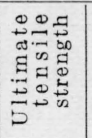 & 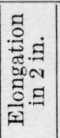 & 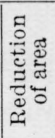 & 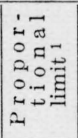 & 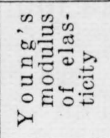 & Purpose & 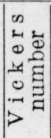 & 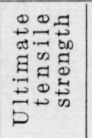 & 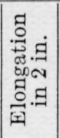 & 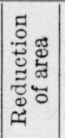 & 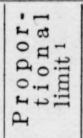 & 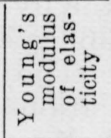 \\
\hline Bolt & $\left\{\begin{array}{l}265 \\
266 \\
269 \\
308\end{array}\right.$ & $\begin{array}{l}\text { lb/in. }{ }^{2} \\
127,900 \\
126,200 \\
128,700 \\
140,800\end{array}$ & \begin{tabular}{|c|}
$\%$ \\
19.0 \\
19.0 \\
20.5 \\
13.5
\end{tabular} & $\begin{array}{l}\% \\
51.8 \\
62.9 \\
61.7 \\
58.9\end{array}$ & $1 \mathrm{~b} / \mathrm{in}^{2}$ & 1b/in. ${ }^{2}$ & Nuts... & $\left\{\begin{array}{l}287 \\
298 \\
307\end{array}\right.$ & $\begin{array}{l}\text { lb/in. }{ }^{2} \\
127,600 \\
130,800 \\
136,100 \\
\end{array}$ & $\begin{array}{l}\% \\
17.0 \\
15.0 \\
16.0\end{array}$ & \begin{tabular}{c|}
$\%$ \\
63.4 \\
59.7 \\
59.2 \\
\end{tabular} & $1 \mathrm{~b} / \mathrm{in}^{2}$ & $\begin{array}{l}1 \mathrm{~b} / \text { in. }^{2} \\
\end{array}$ \\
\hline $\mathrm{Av}_{\text {. }}$ & & 130,900 & 18.0 & 58.8 & ${ }^{2} 25,000$ & $28,500,000$ & & .. & 131,500 & 16.0 & 60.8 & ${ }^{3} 55,900$ & $329,500,000$ \\
\hline
\end{tabular}

Cold-Rolled Steel

\begin{tabular}{|c|c|c|c|c|c|c|c|c|c|c|c|c|c|}
\hline Bolts . & $\left\{\begin{array}{l}202 \\
203 \\
203 \\
205\end{array} \mid\right.$ & $\begin{array}{l}85,600 \\
79,700 \\
85,200 \\
80,800\end{array}$ & $\begin{array}{l}17.5 \\
18.5 \\
18.5 \\
18.5\end{array}$ & $\begin{array}{l}48.9 \\
54.5 \\
50.9 \\
55.7\end{array}$ & 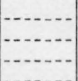 & 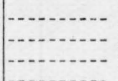 & Nuts.... & $\left\{\begin{array}{l}217 \\
231 \\
254\end{array}\right.$ & $\begin{array}{r}83,600 \\
106,700 \\
104,400\end{array}$ & $\begin{array}{l}18.0 \\
12.0 \\
14.0\end{array}$ & $\begin{array}{l}55.0 \\
34.0 \\
48.3 \\
\end{array}$ & $-\cdots$ & 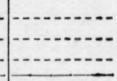 \\
\hline & 211 & 85,100 & 18.0 & 52.7 & - & - & $A v \ldots$ & $\ldots$ & 98,200 & 14. 7 & 45.8 & ${ }^{3} 66,000$ & $329,800,000$ \\
\hline$A v_{.}$. & & 83,300 & 18. 2 & 52.5 & 000 & $230,400,000$ & & & & & & & \\
\hline
\end{tabular}

Monel Metal

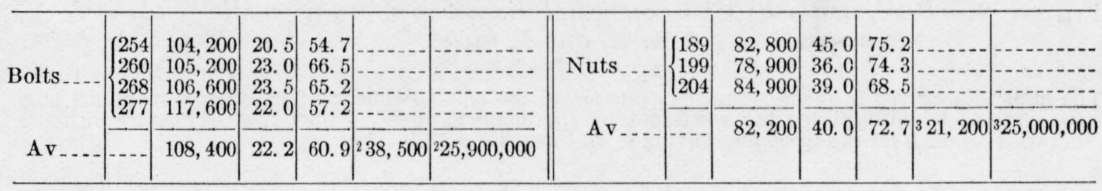

Bronze

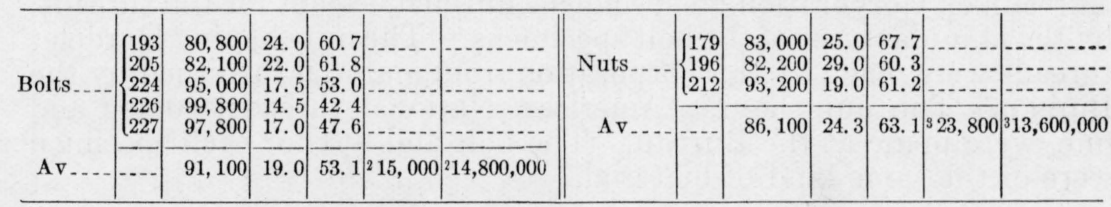

Brass

\begin{tabular}{|c|c|c|c|c|c|c|c|c|c|c|c|c|c|}
\hline Bolts . & $\left\{\begin{array}{l}138 \\
138 \\
141 \\
141\end{array}\right.$ & $\begin{array}{l}57,700 \\
58,800 \\
57,600 \\
60,700\end{array}$ & $\begin{array}{l}25.0 \\
24.5 \\
25.5 \\
22.0\end{array}$ & $\begin{array}{l}46.6 \\
45.4 \\
46.2 \\
37.5\end{array}$ & $\mid \begin{array}{c}\ldots \\
\cdots \\
\cdots\end{array}$ & 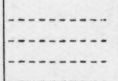 & Nuts.... & $\left\{\begin{array}{l}138 \\
143 \\
162\end{array}\right.$ & $\begin{array}{l}58,400 \\
58,800 \\
68,100\end{array}$ & $\begin{array}{l}27.0 \\
21.0 \\
12.0\end{array}$ & $\begin{array}{l}45.7 \\
49.0 \\
37.8\end{array}$ & 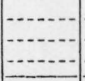 & 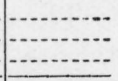 \\
\hline & 147 & 58,700 & 22.0 & 37.2 & - & 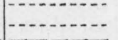 & $A \nabla \ldots$ & $\ldots$ & 61,800 & 20.0 & 44.2 & 318,700 & $311,200,000$ \\
\hline$A v \ldots$ & $\ldots$ & 58,700 & 23.8 & 42.6 & 220,000 & $213,500,000$ & & & & & & & \\
\hline
\end{tabular}

1 Taken as the stress at which the stress-strain curve deviated from a straight line by a strain of 0.00001 in./in.

Determined on 1 specimen, 8 in. gage length.

3 Determined on 1 specimen, 2 in. gage length.

\section{SPECIMENS}

\section{MANUFACTURE}

The bars were sent to a large manufacturer of bolts and nuts who made the bolt specimens. A drawing of the specimens is shown in figure 9 . The threads on the larger end were cut with a die directly in the surface of the bar without any other machine work. The diameter $d$ was the nominal size of the specimen. The portion hav$105145-35-5$ 
ing the diameter $d$ was machined and then threaded. Six similar specimens were made for each of the three types of thread of each of the five materials and for each of the four diameters $(d)$, a total of 360 specimens.

The American National threads were ordered to meet the specifications of class 3, medium fit. ${ }^{9}$ The American National threads, both coarse and fine, on the smaller ends of the specimens were cut by the manufacturer using a single point tool. The threads on the

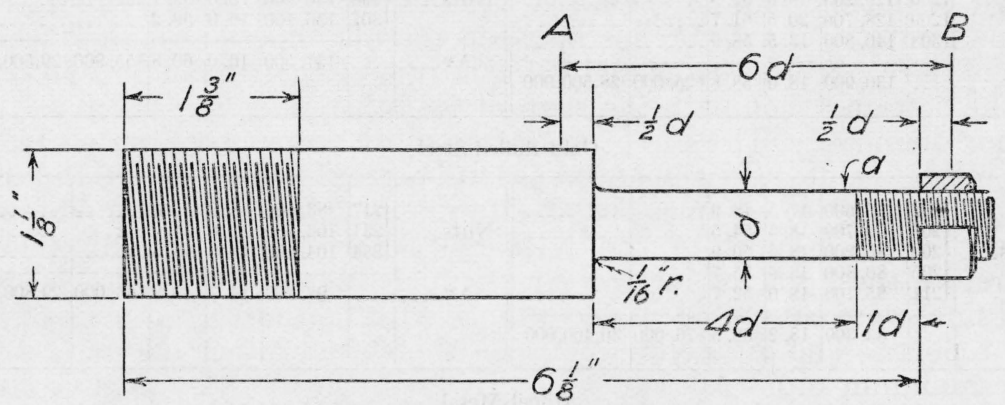

Figure 9.-Specimen bolts whose nominal diameters d at the smaller ends were $3 / 8$, $1 / 2,5 / 8$, and $3 / 4$ inch

The dimensions given in inches were the same for all the bolt specimens. The other dimensions may be obtained by multiplying the coefficient by the nominal thread diameter, $d$. Thus the distance between $A$ and $B$ for the specimens having $1 / 2$ in. threads was $6 \times 1 / 2=3$ in.

smaller ends of the Dardelet bolt specimens were cut by the Dardelet Threadlock Corporation on specimens finished, except for the threads, by the manufacturer of the bolt specimens. The nuts having Dardelet threads were made by this corporation from material furnished by the Bureau. The nuts having American National threads, coarse and fine, were made at the Bureau. The bolt and nut for each specimen were of the same kind of material.

\section{MEASUREMENT OF SPECIMENS}

The results of the measurements on the specimens are given in tables 5 to 9 , inclusive.

\footnotetext{
${ }^{\circ}$ See Report of the National Screw Thread Commission, Misc. Pub. BS M141 (1933).
} 
It was recognized that the strength of bolts depends considerably upon the minor diameter and that the minor diameter may not be within the permissible tolerances even though the pitch diameter is within the tolerances. Each bolt was measured, therefore, and both the minor diameter and the pitch diameter determined. For the minor diameter a Zeiss measuring microscope was used. In this instrument the microscope, having cross hairs in the field, was moved transversely by a screw micrometer having 100 divisions on the barrel of the micrometer. One rotation of the screw moved the microscope $1 \mathrm{~mm}$. The difference between the micrometer reading when one of the cross hairs coincided with the root of the thread on one side of the bolt and then with the root on the other side, was taken as the minor diameter. The minor diameter in inches was computed from the metric value. It is believed that the error in these values did not exceed 0.0004 in. Values for the minimum minor diameter are not given in the Report of the National Screw Thread Commission; therefore these values for the American National bolts, both coarse and fine, were computed in accordance with footnote 1 on page 59 of the report. The differences between the actual minor diameter and the minimum minor diameter are given in the tables. A plus sign indicated that the actual diameter exceeded the minimum diameter and a minus sign that it was less. For the American National bolts the specimens for which this difference is minus did not comply with the requirements for minor diameter. For a few specimens the minor diameter exceeded the maximum allowable minor diameter. These values have been marked with a reference number. These specimens also did not comply with the requirements.

The dimensions of the Dardelet bolts were furnished by the Dardelet Threadlock Corporation. The differences for the minor diameter are the differences between the actual minor diameter and the nominal minor diameter because no tolerances were given.

No pitch diameter was given for the Dardelet threads; therefore, this diameter was not measured.

A screw micrometer having a suitable anvil and point was used for measuring the pitch diameter of the American National bolts. It is believed that the error in these readings did not exceed $0.001 \mathrm{in}$. The values which did not comply with the requirements as to pitch diameter after allowing for this possible error in the reading were marked with a reference number.

Neither the pitch of the bolt nor of the nuts was measured because it was believed that small differences in pitch had little effect upon the tensile strength of the specimens.

For many of the bolts, it was noticed that the axis of the threads on the larger end did not coincide with the axis of the bolt. Each bolt, therefore, was screwed into a threaded holder, on which was mounted a dial micrometer in such a position that rotation of the specimen in the holder indicated the amount of eccentricity of the body of the bolt near the small threads, at a, figure 9 . The position of maximum eccentricity was marked on the bolt. The angle between the axis of the large thread and the axis of the bolt was computed from the readings of the dial. Angles of the order of $0.1 \mathrm{~min}$ of arc could be detected. For some of the bronze and the brass bolts this angle was too small to be measured, being less than this value. 
TABLE 5.-Results of measurements and tests; chromium-nickel steel

\begin{tabular}{|c|c|c|c|c|c|c|c|c|c|c|c|c|c|c|c|c|c|}
\hline 1 & 2 & 3 & 4 & 5 & 6 & 7 & 8 & 8 & 9 & 10 & 11 & 12 & 13 & 14 & 15 & 16 & 17 \\
\hline \multicolumn{18}{|c|}{ 3/8 Inch American National Coarse } \\
\hline \multirow{3}{*}{$\begin{array}{c}\text { Specimen } \\
\text { no. }\end{array}$} & \multicolumn{5}{|c|}{ Bolt } & \multicolumn{3}{|c|}{ Nut } & \multirow{3}{*}{$\begin{array}{c}\text { Clearance } \\
\text { between } \\
\text { nut and } \\
\text { bolt }\end{array}$} & \multicolumn{3}{|c|}{ Impact } & \multicolumn{4}{|c|}{ Static } & \multirow{3}{*}{$\begin{array}{c}\begin{array}{l}\text { Ratio } \\
\text { Impact work (col 12) }\end{array} \\
\text { Static work (col 16) }\end{array}$} \\
\hline & \multirow[b]{2}{*}{$\begin{array}{l}\text { Bar } \\
\text { no. }\end{array}$} & \multirow[b]{2}{*}{$\begin{array}{c}\text { Actual } \\
\text { minor } \\
\text { diameter }\end{array}$} & \multirow{2}{*}{$\begin{array}{c}\text { Differ- } \\
\text { ence be- } \\
\text { tween ac- } \\
\text { tual and } \\
\text { minimum } \\
\text { minor } \\
\text { diameter }\end{array}$} & \multirow[b]{2}{*}{$\begin{array}{l}\text { Pitch } \\
\text { diam- } \\
\text { eter }\end{array}$} & \multirow[b]{2}{*}{$\begin{array}{c}\text { Angle be- } \\
\text { tween axis } \\
\text { of large } \\
\text { thread and } \\
\text { axis of bolt }\end{array}$} & \multirow[b]{2}{*}{$\begin{array}{c}\text { Thick- } \\
\text { ness }\end{array}$} & \multirow{2}{*}{\multicolumn{2}{|c|}{$\begin{array}{l}\text { Obliquity } \\
\text { of bearing } \\
\text { face }\end{array}$}} & & \multirow[b]{2}{*}{$\begin{array}{l}\text { Stretch, } \\
5 \text { diam- } \\
\text { eters }\end{array}$} & \multicolumn{2}{|c|}{ Impact work } & \multirow[b]{2}{*}{$\begin{array}{l}\text { Stretch, } \\
5 \text { diam- } \\
\text { eters }\end{array}$} & \multirow[b]{2}{*}{$\begin{array}{l}\text { Maxi- } \\
\text { mum } \\
\text { load }\end{array}$} & \multicolumn{2}{|c|}{ Static work } & \\
\hline & & & & & & & & & & & $\begin{array}{l}\text { Each } \\
\text { speci- } \\
\text { men }\end{array}$ & $\begin{array}{c}\text { Aver- } \\
\text { age }\end{array}$ & & & $\begin{array}{l}\text { Each } \\
\text { speci- } \\
\text { men }\end{array}$ & $\underset{\text { age }}{\text { A ver- }}$ & \\
\hline $\begin{array}{l}1 \\
1 \\
2 \\
3 \\
4 \\
5 \\
6\end{array}$ & $\begin{array}{l}2 \\
1 \\
4 \\
1 \\
4 \\
1\end{array}$ & \begin{tabular}{c|} 
in. \\
0.2886 \\
.2882 \\
.2858 \\
.2941 \\
.2862 \\
.2902
\end{tabular} & $\begin{array}{l}\text { in. } \\
-0.0019 \\
-.0023 \\
-.0047 \\
+.0036 \\
-.0043 \\
-.0003\end{array}$ & $\begin{array}{l}\text { in. } \\
0.332 \\
.331 \\
.331 \\
.333 \\
.331 \\
.332\end{array}$ & $\begin{array}{c}\operatorname{deg} \\
0.260 \\
.292 \\
.302 \\
.313 \\
.250 \\
.339\end{array}$ & $\begin{array}{l}\text { in. } \\
0.327 \\
.328 \\
.327 \\
.324 \\
.327 \\
.326\end{array}$ & \begin{tabular}{r|}
$\operatorname{deg}$ \\
0 \\
0 \\
0 \\
1 \\
0 \\
0
\end{tabular} & \begin{tabular}{r|}
$\min$ \\
7 \\
7 \\
13 \\
12 \\
52 \\
13
\end{tabular} & \begin{tabular}{l|} 
in. \\
0.0042 \\
.0035 \\
.0050 \\
.0030 \\
.0040 \\
.0037
\end{tabular} & $\begin{array}{c}\text { in. } \\
0.12 \\
.12 \\
.12 \\
.12\end{array}$ & $\begin{array}{r}\text { ft-lb } \\
90 \\
95 \\
90 \\
100 \\
-\end{array}$ & $\begin{array}{r}\mathrm{ft}-\mathrm{lb} \\
94\end{array}$ & $\begin{array}{r}0.10 \\
.10\end{array}$ & $\begin{array}{l}9,370 \\
9,520\end{array}$ & $\mathrm{ft}-\mathrm{lb}$ & ft-lb & 1.21 \\
\hline
\end{tabular}

3/8 Inch American National Fine

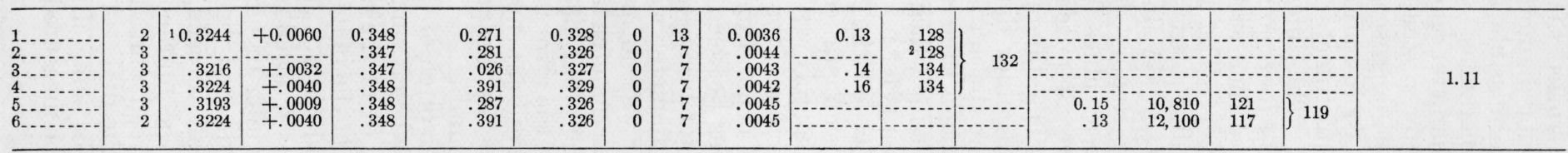


3/8 Inch Dardelet

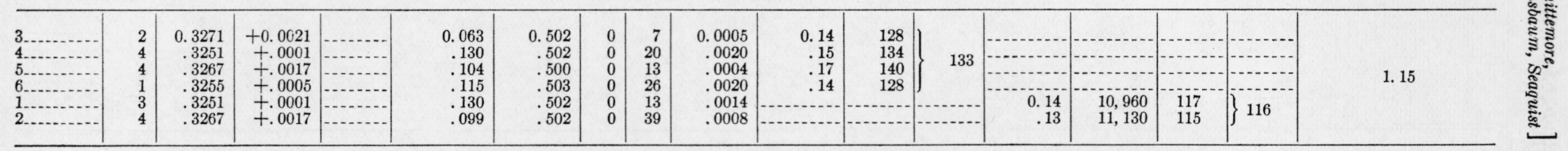

$1 / 2$ Inch American National Coarse

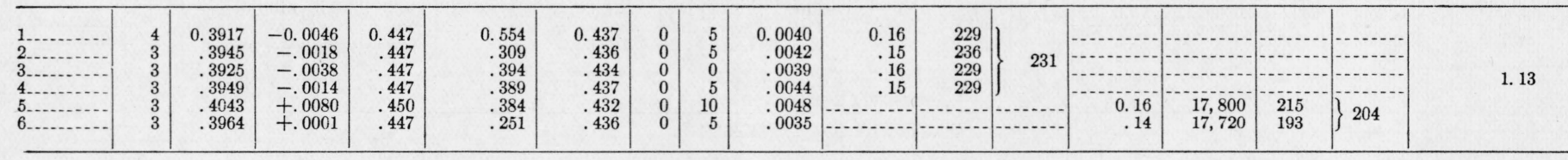

1/2 Inch American National Fine

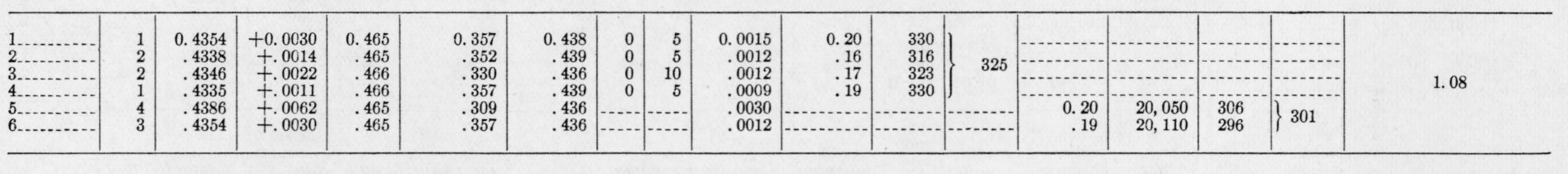

1/2 Inch Dardelet

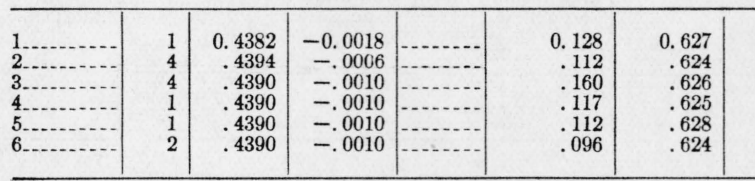

1 Did not comply with requirements.

2 Thread stripped-not included in the average. 
TABLE 5.-Results of measurements and tests; chromium-nickel steel-Continued

\begin{tabular}{|c|c|c|c|c|c|c|c|c|c|c|c|c|c|c|c|c|c|}
\hline 1 & 2 & 3 & 4 & 5 & 8 & 7 & \multicolumn{2}{|c|}{8} & 9 & 10 & 11 & 12 & 13 & 14 & 15 & 16 & 17 \\
\hline \multicolumn{18}{|c|}{ 5/8 Inch American National Coarse } \\
\hline \multirow{3}{*}{$\begin{array}{l}\text { Specimen } \\
\text { no. }\end{array}$} & & \multicolumn{3}{|c|}{ Bolt } & & \multicolumn{3}{|c|}{ Nut } & \multirow{3}{*}{$\begin{array}{c}\text { Clearance } \\
\text { between } \\
\text { nut and } \\
\text { bolt }\end{array}$} & \multicolumn{3}{|c|}{ Impact } & \multicolumn{4}{|c|}{ Static } & \multirow{3}{*}{ 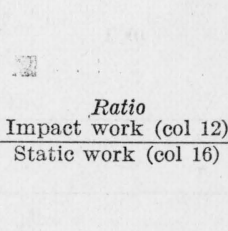 } \\
\hline & \multirow[b]{2}{*}{$\begin{array}{l}\text { Bar } \\
\text { no. }\end{array}$} & \multirow[b]{2}{*}{$\begin{array}{c}\text { Actual } \\
\text { minor } \\
\text { diameter }\end{array}$} & \multirow{2}{*}{$\begin{array}{c}\text { Differ- } \\
\text { ence be- } \\
\text { tween ac- } \\
\text { tual and } \\
\text { minimum } \\
\text { minor } \\
\text { diameter }\end{array}$} & \multirow[b]{2}{*}{$\begin{array}{l}\text { Pitch } \\
\text { diam- } \\
\text { eter }\end{array}$} & \multirow{2}{*}{$\begin{array}{c}\text { Angle be- } \\
\text { tween axis } \\
\text { of large } \\
\text { thread and } \\
\text { axis of bolt }\end{array}$} & \multirow[b]{2}{*}{$\begin{array}{l}\text { Thick- } \\
\text { ness }\end{array}$} & \multirow{2}{*}{\multicolumn{2}{|c|}{$\begin{array}{c}\text { Obliquity } \\
\text { of bearing } \\
\text { face }\end{array}$}} & & \multirow[b]{2}{*}{$\begin{array}{l}\text { Stretch, } \\
5 \text { diam- } \\
\text { eters }\end{array}$} & \multicolumn{2}{|c|}{ Impact work } & \multirow[b]{2}{*}{$\begin{array}{l}\text { Stretch, } \\
5 \text { diam- } \\
\text { eters }\end{array}$} & \multirow[b]{2}{*}{$\begin{array}{l}\text { Maxi- } \\
\text { load }\end{array}$} & \multicolumn{2}{|c|}{ Static work } & \\
\hline & & & & & & & & & & & $\begin{array}{l}\text { Each } \\
\text { speci- } \\
\text { men }\end{array}$ & $\begin{array}{l}\text { Aver- } \\
\text { age }\end{array}$ & & & $\begin{array}{l}\text { Each } \\
\text { speci- } \\
\text { men }\end{array}$ & $\begin{array}{l}\text { Aver- } \\
\text { age }\end{array}$ & \\
\hline $\begin{array}{l}1 \\
2 \\
3 \\
4 \\
5 \\
6\end{array}$ & $\begin{array}{l}4 \\
3 \\
2 \\
2 \\
3 \\
1\end{array}$ & $\begin{array}{l}\text { in. } \\
0.5035 \\
.5016 \\
.5039 \\
.5020 \\
.5079 \\
.5035\end{array}$ & $\begin{array}{c}\text { in. } \\
+0.0007 \\
-.0012 \\
+.0011 \\
-.0008 \\
+.0051 \\
+.0007\end{array}$ & $\begin{array}{l}\text { in. } \\
0.562 \\
.562 \\
.563 \\
.562 \\
.562 \\
.562\end{array}$ & $\begin{array}{c}\operatorname{deg} \\
0.371 \\
.497 \\
.344 \\
.382 \\
.344 \\
.289\end{array}$ & $\begin{array}{l}\text { in. } \\
0.548 \\
.543 \\
.546 \\
.548 \\
.546 \\
.544\end{array}$ & $\begin{array}{r}\operatorname{deg} \\
0 \\
0 \\
0 \\
0 \\
0 \\
0\end{array}$ & \begin{tabular}{r|}
$\min$ \\
8 \\
4 \\
4 \\
0 \\
0 \\
4
\end{tabular} & $\begin{array}{l}\text { in. } \\
0.0055 \\
.0044 \\
.0057 \\
.0051 \\
.0056 \\
.0049\end{array}$ & $\begin{array}{r}\text { in. } \\
0.22 \\
.19 \\
.15 \\
.15 \\
\end{array}$ & $\begin{array}{r}\mathrm{ft}-\mathrm{lb} \\
485 \\
466 \\
426 \\
426 \\
\\
- \\
---\end{array}$ & $\begin{array}{r}\mathrm{ft}-\mathrm{lb} \\
451\end{array}$ & $\begin{array}{r}0.17 \\
.17\end{array}$ & $\begin{array}{l}28,850 \\
28,710\end{array}$ & ft-lb & $\begin{array}{l}\text { ft-lb } \\
\} \\
383\end{array}$ & 1.18 \\
\hline \multicolumn{18}{|c|}{ 5/8 Inch American National Fine } \\
\hline $\begin{array}{l}1 \\
2 \\
3 \\
4 \\
5 \\
5 \\
6\end{array}$ & $\begin{array}{l}3 \\
3 \\
4 \\
3 \\
3 \\
1\end{array}$ & $\begin{array}{r}0.5500 \\
.5500 \\
1.5579 \\
.5512 \\
.5516\end{array}$ & $\begin{array}{r}+0.0002 \\
+.0002 \\
+.0019 \\
+.0014 \\
+.0018\end{array}$ & $\begin{array}{r}0.586 \\
.586 \\
.586 \\
.587 \\
.587 \\
.588\end{array}$ & $\begin{array}{r}0.229 \\
.284 \\
.459 \\
.491 \\
.349 \\
.448\end{array}$ & $\begin{array}{r}0.545 \\
.546 \\
.546 \\
.546 \\
.545 \\
.547\end{array}$ & $\begin{array}{l}0 \\
0 \\
0 \\
0 \\
0 \\
0\end{array}$ & \begin{tabular}{r|}
15 \\
4 \\
4 \\
0 \\
19 \\
4
\end{tabular} & $\begin{array}{l}0.0043 \\
.0047 \\
.0053 \\
.0046 \\
.0046 \\
.0058\end{array}$ & \begin{tabular}{r}
0.24 \\
.25 \\
.25 \\
\hdashline-2 \\
...-
\end{tabular} & $\begin{array}{r}2362 \\
683 \\
683 \\
720\end{array}$ & 695 & $\begin{array}{l}0.25 \\
.25\end{array}$ & $\begin{array}{l}32,320 \\
32,670\end{array}$ & $\begin{array}{l}622 \\
624\end{array}$ & 623 & 1.12 \\
\hline \multicolumn{18}{|c|}{$5 / 8$ Inch Dardelet } \\
\hline $\begin{array}{l}1 \\
2 \\
3 \\
4 \\
5 \\
6\end{array}$ & $\begin{array}{l}3 \\
3 \\
2 \\
4 \\
2 \\
2\end{array}$ & $\begin{array}{l}0.5512 \\
.5504 \\
.5500 \\
.5502 \\
.5498 \\
.5507\end{array}$ & $\begin{array}{r}+0.0012 \\
+.0004 \\
.0000 \\
+.0002 \\
-.0002 \\
+.0007\end{array}$ & 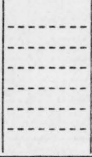 & $\begin{array}{r}0.131 \\
.115 \\
.087 \\
.109 \\
.126 \\
.115\end{array}$ & $\begin{array}{l}0.749 \\
.750 \\
.750 \\
.749 \\
.752 \\
.751\end{array}$ & $\begin{array}{l}0 \\
0 \\
0 \\
0 \\
0 \\
0\end{array}$ & $\begin{array}{r}15 \\
15 \\
11 \\
11 \\
8 \\
8\end{array}$ & $\begin{array}{l}0.0023 \\
.0028 \\
.0016 \\
.0013 \\
.0010 \\
.0012\end{array}$ & $\begin{array}{r}0.25 \\
.26 \\
.21 \\
.25 \\
-. .-\end{array}$ & \begin{tabular}{r}
609 \\
637 \\
555 \\
618 \\
\hdashline.-- \\
\end{tabular} & 605 & $\begin{array}{l}0.17 \\
.16\end{array}$ & $\begin{array}{l}35,000 \\
36,070\end{array}$ & $\begin{array}{l}496 \\
469\end{array}$ & 482 & 1. 26 \\
\hline
\end{tabular}


34 Inch American National Coarse

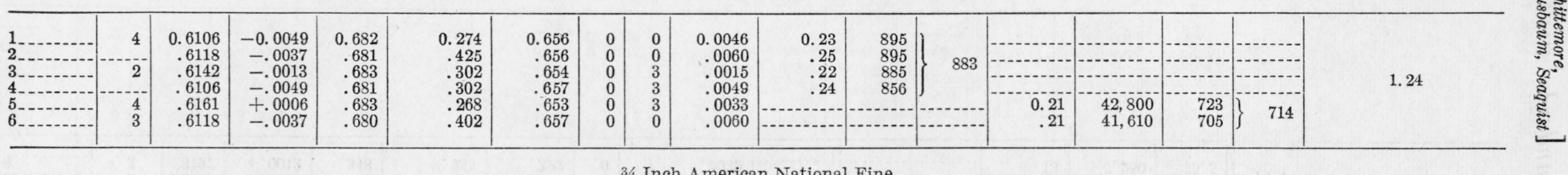

3/4 Inch American National Fine

\begin{tabular}{|c|c|c|c|c|c|c|c|c|c|c|c|c|c|c|c|c|c|}
\hline $\begin{array}{l}1 \\
2 \\
3 \\
5 \\
6\end{array}$ & $\begin{array}{l}4 \\
2 \\
2 \\
4 \\
2 \\
1\end{array}$ & $\begin{array}{l}0.6709 \\
.6665 \\
.6705 \\
.6713 \\
.6705 \\
.6720\end{array}$ & $\begin{array}{r}+0.0053 \\
+.0009 \\
+.0049 \\
+.0057 \\
+.0049 \\
+.0064\end{array}$ & $\begin{array}{r}0.709 \\
.706 \\
.707 \\
.708 \\
.707 \\
.708\end{array}$ & $\begin{array}{r}0.369 \\
.582 \\
.268 \\
.632 \\
.291 \\
.408\end{array}$ & $\begin{array}{r}0.656 \\
.650 \\
.653 \\
.658 \\
.657 \\
.658\end{array}$ & $\begin{array}{l}0 \\
0 \\
0 \\
0 \\
0 \\
0\end{array}$ & $\begin{array}{l}7 \\
3 \\
3 \\
0 \\
0 \\
0\end{array}$ & $\begin{array}{r}0.0058 \\
.0033 \\
.0035 \\
.0040 \\
.0052 \\
.0035\end{array}$ & $\begin{array}{r}0.31 \\
.29 \\
.27 \\
.31 \\
\hdashline \\
\hdashline\end{array}$ & $\begin{array}{l}1,298 \\
1,201 \\
1,194 \\
1,270\end{array}$ & 1,241 & $\begin{array}{r}0.22 \\
.30\end{array}$ & $\begin{array}{l}53,500 \\
48,310\end{array}$ & $\begin{array}{r}923 \\
1,130\end{array}$ & 1,026 & 1. 21 \\
\hline
\end{tabular}

3/4 Inch Dardelet

\begin{tabular}{|c|c|c|c|c|c|c|c|c|c|c|c|c|c|c|c|c|c|}
\hline $\begin{array}{l}1 \\
2 \\
3 \\
4 \\
5\end{array}$ & $\begin{array}{r}2 \\
4 \\
1 \\
-4\end{array}$ & $\begin{array}{r}0.6775 \\
.6772 \\
.6762 \\
.6772 \\
.6756 \\
.6750\end{array}$ & $\begin{array}{r}+0.0025 \\
+.0022 \\
+.0012 \\
+.0022 \\
+.0006 \\
.0000\end{array}$ & 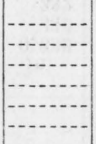 & $\begin{array}{l}0.095 \\
.123 \\
.106 \\
.123 \\
.117\end{array}$ & $\begin{array}{r}0.874 \\
.874 \\
.873 \\
.874 \\
.873 \\
.882\end{array}$ & $\begin{array}{l}0 \\
0 \\
0 \\
0 \\
0 \\
-\end{array}$ & $\begin{array}{r}10 \\
7 \\
13 \\
17 \\
13\end{array}$ & $\begin{array}{l}0.0006 \\
.0005 \\
.0019 \\
.0015 \\
.0007\end{array}$ & $\begin{array}{r}0.26 \\
.30 \\
.32 \\
.31 \\
\\
\end{array}$ & $\begin{array}{l}1,132 \\
1,260 \\
1,240 \\
1,231\end{array}$ & 1,216 & $\begin{array}{r}0.25 \\
.24\end{array}$ & $\begin{array}{l}47,800 \\
47,930\end{array}$ & $\begin{array}{l}998 \\
926\end{array}$ & 962 & 1. 26 \\
\hline
\end{tabular}


TABLE 6.-Results of measurements and tests: cold-rolled steel

\begin{tabular}{|c|c|c|c|c|c|c|c|c|c|c|c|c|c|c|c|c|c|}
\hline 1 & 2 & 3 & 4 & 5 & 6 & 7 & & 8 & 9 & 10 & 11 & 12 & 13 & 14 & 15 & 16 & 17 \\
\hline & \multicolumn{17}{|c|}{ 3/8 Inch American National Coarse } \\
\hline \multirow{3}{*}{$\begin{array}{l}\text { Specimen } \\
\text { no. }\end{array}$} & \multirow{3}{*}{$\begin{array}{l}\text { Bar } \\
\text { no. }\end{array}$} & \multicolumn{4}{|c|}{ Bolt } & \multicolumn{3}{|c|}{ Nut } & & \multicolumn{3}{|c|}{ Impact } & \multicolumn{4}{|c|}{ Static } & \multirow{3}{*}{$\begin{array}{c}\text { Ratio } \\
\text { Impact work (col. 12) } \\
\text { Static work (col. 16) }\end{array}$} \\
\hline & & \multirow[b]{2}{*}{$\begin{array}{c}\text { Actual } \\
\text { minor } \\
\text { diamete }\end{array}$} & \multirow{2}{*}{$\begin{array}{c}\text { Differ- } \\
\text { ence be- } \\
\text { tween ac- } \\
\text { tual and } \\
\text { minimum } \\
\text { minor } \\
\text { diameter }\end{array}$} & \multirow{2}{*}{$\begin{array}{l}\text { Pitch } \\
\text { diam- } \\
\text { eter }\end{array}$} & \multirow{2}{*}{$\begin{array}{l}\text { Angle be- } \\
\text { tween axis } \\
\text { of large } \\
\text { thread and } \\
\text { axis of bolt }\end{array}$} & \multirow[b]{2}{*}{$\begin{array}{l}\text { Thick- } \\
\text { ness }\end{array}$} & \multirow{2}{*}{\multicolumn{2}{|c|}{$\begin{array}{c}\text { Obliquity } \\
\text { of bearing } \\
\text { face }\end{array}$}} & \multirow{2}{*}{$\begin{array}{c}\text { Clearance } \\
\text { between } \\
\text { nut and } \\
\text { bolt }\end{array}$} & \multicolumn{3}{|c|}{ Impact work } & \multirow[b]{2}{*}{$\begin{array}{l}\text { Stretch, } \\
5 \text { diam- } \\
\text { eters }\end{array}$} & \multirow{2}{*}{$\underset{\text { Maxi- }}{\text { mum }}$} & \multicolumn{2}{|c|}{ Static work } & \\
\hline & & & & & & & & & & $\begin{array}{l}\text { Stretch, } \\
5 \text { diam- } \\
\text { eters }\end{array}$ & $\begin{array}{l}\text { Each } \\
\text { speci- } \\
\text { men }\end{array}$ & $\begin{array}{c}\text { A ver- } \\
\text { age }\end{array}$ & & & $\begin{array}{l}\text { Each } \\
\text { speci- } \\
\text { men }\end{array}$ & $\begin{array}{l}\text { A ver- } \\
\text { age }\end{array}$ & \\
\hline 1. & 4 & $\begin{array}{l}\text { in. } \\
0.2862\end{array}$ & $\begin{array}{c}\text { in. } \\
-0.0043\end{array}$ & $\begin{array}{l}\text { in. } \\
0.332\end{array}$ & $\stackrel{\operatorname{deg}}{0.099}$ & $\begin{array}{l}\text { in. } \\
0.327\end{array}$ & $\operatorname{deg}$ & $\min _{7}$ & $\begin{array}{l}\text { in. } \\
0.0051\end{array}$ & in. 0.10 & $\begin{array}{l}\mathrm{ft}-\mathrm{lb} \\
\quad 70\end{array}$ & ft-lb & in. & lb & ft-lb & $\mathrm{ft}-\mathrm{lb}$ & \\
\hline 3 & 4 & .2858 & -.0047 & .332 & .271 & .327 & 0 & 7 & .0053 & .10 & 64 & 68 & & & 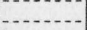 & & \\
\hline 3 & $\begin{array}{l}2 \\
5\end{array}$ & $\begin{array}{l}.2866 \\
.2866\end{array}$ & $\begin{array}{l}-.0039 \\
-.0039\end{array}$ & .332 & $\begin{array}{l}.240 \\
.177\end{array}$ & .327 & $\begin{array}{l}0 \\
0\end{array}$ & $\begin{array}{l}0 \\
7\end{array}$ & .0046 & .11 & 70 & & & & & & 1.34 \\
\hline 5 & 5 & .2866 & $\begin{array}{l}-.0039 \\
-.0039\end{array}$ & .331 & .208 & $\begin{array}{l}.320 \\
.326\end{array}$ & $\begin{array}{l}0 \\
0\end{array}$ & 0 & .0060 & & & & 0.10 & 6,370 & 50.9 & & \\
\hline 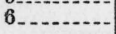 & 2 & .2854 & -.0051 & .331 & .438 & .325 & 0 & 7 & .0059 & & & & .10 & 6,630 & 50.8 & 50.8 & \\
\hline
\end{tabular}

3/8 Inch American National Fine

\begin{tabular}{|c|c|c|c|c|c|c|c|c|c|c|c|c|c|c|c|c|c|}
\hline $\begin{array}{l}1 \\
2 \\
5 \\
5\end{array}$ & $\begin{array}{l}2 \\
3 \\
5 \\
1 \\
5 \\
3\end{array}$ & $\begin{array}{r}0.3205 \\
.3201 \\
.3193 \\
.3205 \\
.3197 \\
.3197\end{array}$ & $\begin{array}{r}+0.0021 \\
+.0017 \\
+.0009 \\
+.0021 \\
+.0013 \\
+.0013\end{array}$ & $\begin{array}{r}0.348 \\
.348 \\
.348 \\
.348 \\
.348 \\
.348\end{array}$ & $\begin{array}{r}0.073 \\
.182 \\
.156 \\
.276 \\
.250 \\
.255\end{array}$ & $\begin{array}{l}0.325 \\
.328 \\
.322 \\
.323 \\
.323 \\
.322\end{array}$ & $\begin{array}{l}0 \\
0 \\
0 \\
0 \\
0 \\
0\end{array}$ & $\begin{array}{l}7 \\
0 \\
0 \\
7 \\
0 \\
7\end{array}$ & $\begin{array}{l}0.0031 \\
.0031 \\
.0039 \\
.0040 \\
.0028 \\
.0039\end{array}$ & $\begin{array}{r}0.13 \\
.14 \\
.14 \\
.13 \\
\end{array}$ & $\begin{array}{l}90 \\
95 \\
95 \\
90\end{array}$ & 92 & $\begin{array}{r}0.13 \\
.13\end{array}$ & $\begin{array}{l}7,520 \\
7,250\end{array}$ & $\begin{array}{l}75.2 \\
75.5\end{array}$ & 75.4 & 1. 22 \\
\hline
\end{tabular}




\begin{tabular}{|c|c|c|c|c|c|c|c|c|c|c|c|c|c|c|c|c|c|}
\hline & $\begin{array}{l} \\
1 \\
1 \\
3 \\
1 \\
2 \\
4\end{array}$ & $\begin{array}{r}0.3251 \\
.3255 \\
.3263 \\
.3255 \\
.3255 \\
.3255\end{array}$ & $\begin{array}{r}+0.0001 \\
+.0005 \\
+.0013 \\
+.0005 \\
+.0005 \\
+.0005\end{array}$ & $\mid$\begin{tabular}{l} 
\\
\hdashline
\end{tabular} & $\begin{array}{r}0.109 \\
.161 \\
.104 \\
.083 \\
.099 \\
.156\end{array}$ & $\begin{array}{l}0.500 \\
.498 \\
.501 \\
.499 \\
.499 \\
.496\end{array}$ & \begin{tabular}{l|} 
\\
0 \\
0 \\
0 \\
0 \\
0 \\
0
\end{tabular} & \begin{tabular}{l|} 
\\
33 \\
26 \\
33 \\
26 \\
33 \\
26
\end{tabular} & $\begin{array}{l}0.0012 \\
.0005 \\
.0008 \\
.0004 \\
.0007 \\
.0008\end{array}$ & $\begin{array}{r}0.14 \\
.18 \\
.16 \\
.15 \\
\end{array}$ & $\begin{array}{r}95 \\
100 \\
95 \\
90 \\
\hdashline-.-\end{array}$ & 95 & $\begin{array}{r} \\
0.13 \\
.14\end{array}$ & $\begin{array}{l}7,70 \\
7,920 \\
7,210\end{array}$ & $\begin{array}{l}82.4 \\
80.5\end{array}$ & \begin{tabular}{|c|} 
\\
\hdashline 81.4
\end{tabular} & 1.17 \\
\hline
\end{tabular}

1/2 Inch American National Coarse

\begin{tabular}{|c|c|c|c|c|c|c|c|c|c|c|c|c|c|c|c|c|c|}
\hline $\begin{array}{l}1 \\
4 \\
5 \\
5\end{array}$ & $\begin{array}{l}2 \\
4 \\
2 \\
3 \\
1 \\
3\end{array}$ & $\begin{array}{l}0.3921 \\
.3976 \\
.3964 \\
.3898 \\
.3976 \\
.3984\end{array}$ & $\begin{array}{r}-0.0042 \\
+.0013 \\
+.0001 \\
-.0065 \\
+.0013 \\
+.0021\end{array}$ & $\begin{array}{r}0.447 \\
1.452 \\
1.451 \\
.447 \\
1.451 \\
1.452\end{array}$ & $\begin{array}{l}0.304 \\
.245 \\
.373 \\
.261 \\
.250 \\
.426\end{array}$ & $\begin{array}{r}0.438 \\
.437 \\
.437 \\
.438 \\
.439 \\
.436\end{array}$ & $\begin{array}{l}0 \\
0 \\
0 \\
0 \\
0 \\
0\end{array}$ & $\begin{array}{r}5 \\
5 \\
0 \\
10 \\
14 \\
5\end{array}$ & $\begin{array}{r}0.0055 \\
.0015 \\
.0018 \\
.0060 \\
.0013 \\
.0003\end{array}$ & $\begin{array}{r}0.03 \\
.15 \\
.04 \\
.03 \\
\end{array}$ & $\begin{array}{r}250 \\
164 \\
260 \\
250 \\
\\
\end{array}$ & 164 & $\begin{array}{r}0.12 \\
.13\end{array}$ & $\begin{array}{l}12,360 \\
11,690\end{array}$ & $\begin{array}{l}116 \\
121\end{array}$ & 118 & 1. 39 \\
\hline
\end{tabular}

1/2 Inch American National Fine

\begin{tabular}{|c|c|c|c|c|c|c|c|c|c|c|c|c|c|c|c|c|c|}
\hline $\begin{array}{l}1 \\
2 \\
4 \\
5 \\
6\end{array}$ & $\begin{array}{l}3 \\
1 \\
4 \\
5 \\
3 \\
4\end{array}$ & $\begin{array}{l}0.4346 \\
.4303 \\
.4287 \\
.4342 \\
.4346 \\
.4338\end{array}$ & $\begin{array}{r}+0.0022 \\
-.0021 \\
-.0037 \\
+.0018 \\
+.0022 \\
+.0014\end{array}$ & $\begin{array}{r}0.466 \\
.466 \\
.466 \\
.466 \\
.466 \\
.466\end{array}$ & $\begin{array}{l}0.240 \\
.293 \\
.224 \\
.261 \\
.272 \\
.298\end{array}$ & $\begin{array}{r}0.439 \\
.429 \\
.437 \\
.433 \\
.433 \\
.433\end{array}$ & $\begin{array}{l}0 \\
0 \\
0 \\
0 \\
0 \\
0\end{array}$ & $\begin{array}{r}5 \\
5 \\
5 \\
5 \\
10 \\
5\end{array}$ & $\begin{array}{l}0.0044 \\
.0009 \\
.0012 \\
.0011 \\
.0011 \\
.0012\end{array}$ & $\begin{array}{r}0.19 \\
.17 \\
.19 \\
.18 \\
\\
\end{array}$ & $\begin{array}{l}229 \\
216 \\
223 \\
236\end{array}$ & $\mid$ & $\begin{array}{l}0.17 \\
.18\end{array}$ & $\begin{array}{l}13,300 \\
13,020\end{array}$ & $\begin{array}{l}182 \\
178\end{array}$ & 180 & 1. 26 \\
\hline
\end{tabular}

1/2 Inch Dardelet

\begin{tabular}{|c|c|c|c|c|c|c|c|c|c|c|c|c|c|c|c|c|c|}
\hline $\begin{array}{l}3 \\
6 \\
6 \\
1\end{array}$ & $\begin{array}{l}5 \\
1 \\
5 \\
1 \\
2 \\
4\end{array}$ & $\begin{array}{r}0.4386 \\
.4390 \\
.4377 \\
.4402 \\
.4386 \\
.4386\end{array}$ & $\begin{array}{r}-0.0014 \\
-.0010 \\
-.0023 \\
+.0002 \\
-.0014 \\
-.0014\end{array}$ & 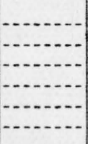 & $\begin{array}{r}0.101 \\
.091 \\
.080 \\
.101 \\
.101 \\
.117\end{array}$ & $\begin{array}{r}0.627 \\
.626 \\
.627 \\
.628 \\
.626 \\
.627\end{array}$ & $\begin{array}{l}0 \\
0 \\
0 \\
0 \\
0 \\
0\end{array}$ & $\begin{array}{l}14 \\
19 \\
10 \\
10 \\
10 \\
14\end{array}$ & $\begin{array}{l}0.0028 \\
.0014 \\
.0016 \\
.0011 \\
.0008 \\
.0030\end{array}$ & $\begin{array}{r}0.20 \\
.21 \\
.21 \\
.20 \\
-\end{array}$ & $\begin{array}{l}236 \\
250 \\
250 \\
236 \\
-\end{array}$ & 243 & $\begin{array}{r}0.16 \\
.17\end{array}$ & $\begin{array}{l}14,150 \\
13,030\end{array}$ & $\begin{array}{l}187 \\
175\end{array}$ & 181 & 1. 34 \\
\hline
\end{tabular}

1 Did not comply with requirements.

2 Brittle failure-not included in the average. 
TABLE 6.-Results of measurements and tests: cold-rolled steel-Continued

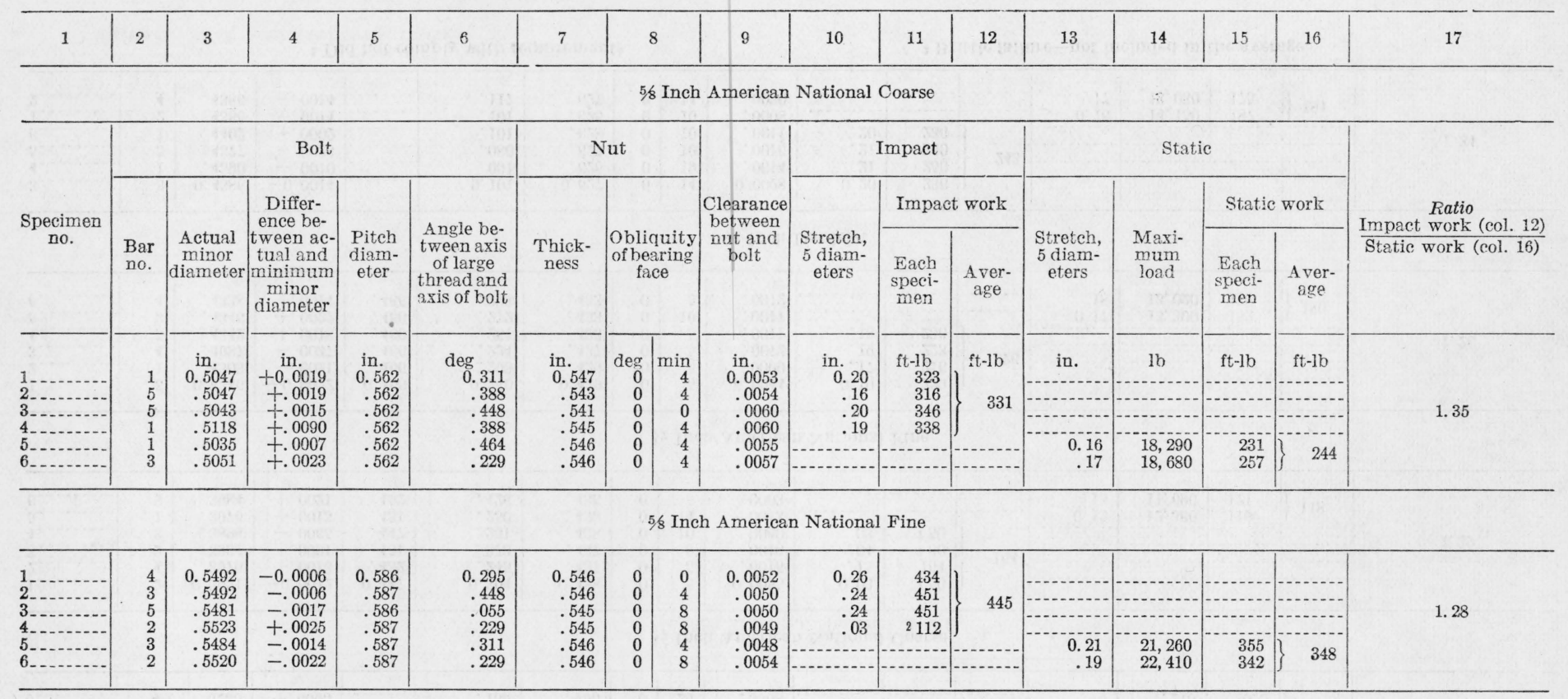


5/8 Inch Dardelet

\begin{tabular}{|c|c|c|c|c|c|c|c|c|c|c|c|c|c|c|c|c|c|}
\hline (5) & \begin{tabular}{l|l}
4 \\
4 \\
2 \\
1 \\
2 \\
5
\end{tabular} & $\begin{array}{r}0.5498 \\
.5507 \\
.5500 \\
.5512 \\
.5512 \\
.5500\end{array}$ & $\begin{array}{r}-0.0002 \\
+.0007 \\
.0000 \\
+.0012 \\
+.0012 \\
.0000\end{array}$ & 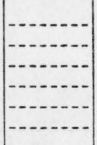 & $\begin{array}{l}0.142 \\
.131 \\
.071 \\
.098 \\
.082 \\
.126\end{array}$ & $\begin{array}{r}0.750 \\
.752 \\
.751 \\
.753 \\
.749 \\
.749\end{array}$ & $\begin{array}{l}0 \\
0 \\
0 \\
0 \\
0 \\
0\end{array}$ & \begin{tabular}{r|r|}
4 \\
8 \\
8 \\
11 \\
11 \\
15
\end{tabular} & $\begin{array}{l}0.0027 \\
.0016 \\
.0026 \\
.0012 \\
.0024 \\
.0005\end{array}$ & $\begin{array}{r}0.25 \\
.26 \\
.23 \\
.25 \\
-. . .\end{array}$ & $\begin{array}{l}418 \\
460 \\
418 \\
443 \\
-\end{array}$ & 435 & $\begin{array}{r}0.19 \\
.17\end{array}$ & $\begin{array}{l}22,020 \\
22,000\end{array}$ & $\begin{array}{l}343 \\
317\end{array}$ & 330 & 1. 32 \\
\hline
\end{tabular}

3/4 Inch American National Coarse

\begin{tabular}{|c|c|c|c|c|c|c|c|c|c|c|c|c|c|c|c|c|c|}
\hline (1. & $\begin{array}{l}4 \\
1 \\
5 \\
2 \\
1 \\
1\end{array}$ & $\begin{array}{r}0.6121 \\
.6114 \\
.6122 \\
.6088 \\
.6118 \\
.6118\end{array}$ & $\begin{array}{r}-0.0034 \\
-.0041 \\
-.0033 \\
-.0067 \\
-.0037 \\
-.0037\end{array}$ & $\begin{array}{r}0.682 \\
.682 \\
.681 \\
.681 \\
.681 \\
.682\end{array}$ & $\begin{array}{l}0.456 \\
.497 \\
.520 \\
.274 \\
.235 \\
.553\end{array}$ & $\begin{array}{r}0.653 \\
.655 \\
.655 \\
.655 \\
.652 \\
.652\end{array}$ & $\begin{array}{l}0 \\
0 \\
0 \\
0 \\
0 \\
0\end{array}$ & $\begin{array}{l}3 \\
3 \\
3 \\
0 \\
0 \\
3\end{array}$ & $\begin{array}{l}0.0042 \\
.0049 \\
.0057 \\
.0052 \\
.0043 \\
.0053\end{array}$ & $\begin{array}{l}0.25 \\
.03 \\
.03 \\
.03\end{array}$ & $\begin{array}{r}564 \\
290 \\
2106 \\
279\end{array}$ & 564 & $\begin{array}{r}0.19 \\
.18\end{array}$ & $\begin{array}{l}28,860 \\
28,800\end{array}$ & $\begin{array}{l}438 \\
426\end{array}$ & 432 & 1. 31 \\
\hline
\end{tabular}

3/4 Inch American National Fine

\begin{tabular}{|c|c|c|c|c|c|c|c|c|c|c|c|c|c|c|c|c|c|}
\hline $\begin{array}{l}1 \\
2 \ldots \\
3-\ldots \\
4-\ldots \\
6 \\
\end{array}$ & $\begin{array}{c}2 \\
-4 \\
1 \\
3 \\
3\end{array}$ & $\begin{array}{r}0.6630 \\
.6643 \\
.6634 \\
.6639 \\
.6642 \\
.6630\end{array}$ & $\begin{array}{r}-0.0026 \\
-.0013 \\
-.0022 \\
-.0017 \\
-.0014 \\
-.0026\end{array}$ & $\begin{array}{r}0.707 \\
.708 \\
.707 \\
.707 \\
.707 \\
.708\end{array}$ & $\begin{array}{l}0.212 \\
.514 \\
.553 \\
.693 \\
.358 \\
.564\end{array}$ & $\begin{array}{r}0.656 \\
.650 \\
.655 \\
.656 \\
.650 \\
.655\end{array}$ & $\begin{array}{l}0 \\
0 \\
0 \\
0 \\
0 \\
0\end{array}$ & $\begin{array}{l}7 \\
0 \\
3 \\
7 \\
3 \\
3\end{array}$ & $\begin{array}{l}0.0038 \\
.0037 \\
.0042 \\
.0045 \\
.0041 \\
.0045\end{array}$ & $\begin{array}{r}0.14 \\
.27 \\
.10 \\
.05\end{array}$ & $\begin{array}{r}2418 \\
768 \\
2278 \\
2128 \\
-\end{array}$ & 768 & $\begin{array}{r}0.25 \\
.26\end{array}$ & $\begin{array}{l}31,420 \\
31,270\end{array}$ & $\begin{array}{l}611 \\
620\end{array}$ & 616 & 1. 25 \\
\hline
\end{tabular}

3/4 Inch Dardelet

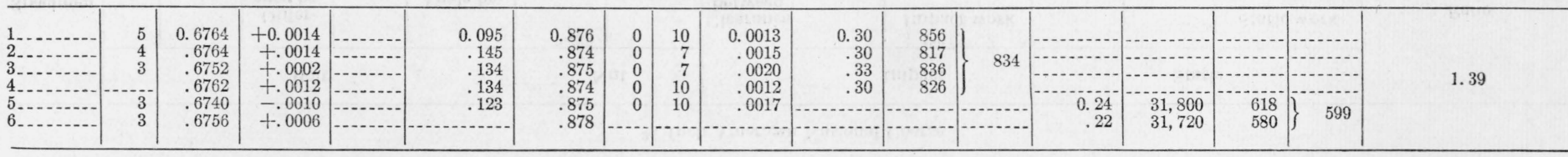

${ }^{2}$ Brittle failure-not included in the average. 
TABLE 7.-Results of measurements and tests: monel metal

\begin{tabular}{|c|c|c|c|c|c|c|c|c|c|c|c|c|c|c|c|c|c|}
\hline 1 & 2 & 3 & 4 & 5 & 6 & 7 & \multicolumn{2}{|c|}{8} & 9 & 10 & 11 & 12 & 13 & 14 & 15 & 16 & 17 \\
\hline \multicolumn{18}{|c|}{ 3/8 Inch American National Coarse } \\
\hline \multirow{3}{*}{$\begin{array}{l}\text { Specimen } \\
\text { no. }\end{array}$} & \multicolumn{5}{|c|}{ Bolt } & \multicolumn{3}{|c|}{ Nut } & \multirow{3}{*}{$\begin{array}{c}\text { Clearance } \\
\text { between } \\
\text { nut and } \\
\text { bolt }\end{array}$} & \multicolumn{3}{|c|}{ Impact } & \multicolumn{4}{|c|}{ Static } & \multirow{3}{*}{$\frac{\text { Ratio }}{\text { Impact work (col. 12) }}$} \\
\hline & \multirow[b]{2}{*}{$\begin{array}{l}\text { Bar } \\
\text { no. }\end{array}$} & \multirow[b]{2}{*}{$\begin{array}{c}\text { Actual } \\
\text { minor } \\
\text { diameter }\end{array}$} & \multirow{2}{*}{$\begin{array}{c}\text { Differ- } \\
\text { ence be- } \\
\text { tween ac- } \\
\text { tual and } \\
\text { minimum } \\
\text { minor } \\
\text { diameter }\end{array}$} & \multirow[b]{2}{*}{$\begin{array}{c}\text { Pitch } \\
\text { diam- } \\
\text { eter }\end{array}$} & \multirow{2}{*}{$\begin{array}{c}\text { Angle be- } \\
\text { tween axis } \\
\text { of large } \\
\text { thread and } \\
\text { axis of bolt }\end{array}$} & \multirow[b]{2}{*}{$\begin{array}{l}\text { Thick- } \\
\text { ness }\end{array}$} & \multirow{2}{*}{\multicolumn{2}{|c|}{$\begin{array}{c}\text { Obliquity } \\
\text { of bearing } \\
\text { face }\end{array}$}} & & \multirow{2}{*}{$\begin{array}{l}\text { Stretch, } \\
5 \text { diam- } \\
\text { eters }\end{array}$} & \multicolumn{2}{|c|}{ Impact work } & \multirow[b]{2}{*}{$\begin{array}{l}\text { Stretch, } \\
5 \text { diam- } \\
\text { eters }\end{array}$} & \multirow[b]{2}{*}{$\underset{\text { muxi- }}{\text { Mad }}$} & \multicolumn{2}{|c|}{ Static work } & \\
\hline & & & & & & & & & & & $\begin{array}{c}\text { Each } \\
\text { speci- } \\
\text { men }\end{array}$ & $\begin{array}{l}\text { A ver- } \\
\text { age }\end{array}$ & & & $\begin{array}{l}\text { Each } \\
\text { speci- } \\
\text { men }\end{array}$ & $\begin{array}{l}\text { A ver- } \\
\text { age }\end{array}$ & \\
\hline $\begin{array}{l}1 \\
2 \\
3 \\
4 \\
5\end{array}$ & $\begin{array}{l}2 \\
3 \\
3 \\
3 \\
3 \\
3\end{array}$ & $\begin{array}{c}\text { in. } \\
0.2874 \\
.2874 \\
.2886 \\
.2870 \\
.2862 \\
.2882\end{array}$ & $\begin{array}{c}\text { in. } \\
-0.0031 \\
-.0031 \\
-.0019 \\
-.0035 \\
-.0043 \\
-.0023\end{array}$ & $\begin{array}{c}\text { in. } \\
0.332 \\
.333 \\
.332 \\
.332 \\
.332 \\
.332\end{array}$ & $\begin{array}{c}\operatorname{deg} \\
0.354 \\
.313 \\
.313 \\
.386 \\
.469 \\
.344\end{array}$ & $\begin{array}{l}\text { in. } \\
0.323 \\
.328 \\
.326 \\
.333 \\
.336 \\
.326\end{array}$ & $\begin{array}{r}\operatorname{deg} \\
0 \\
0 \\
0 \\
0 \\
0 \\
0\end{array}$ & $\begin{array}{r}\min \\
13 \\
13 \\
7 \\
39 \\
52 \\
39\end{array}$ & $\begin{array}{l}\text { in. } \\
0.0018 \\
.0021 \\
.0013 \\
.0011 \\
.0012 \\
.0015\end{array}$ & $\begin{array}{r}\text { in. } \\
0.14 \\
.14 \\
.14 \\
.14 \\
\\
\end{array}$ & $\begin{array}{r}\mathrm{ft}-\mathrm{lb} \\
100 \\
100 \\
100 \\
100 \\
\hdashline \\
\hdashline\end{array}$ & $\begin{array}{r}\text { ft-lb } \\
100\end{array}$ & $\begin{array}{r}0.13 \\
.12\end{array}$ & $\begin{array}{r}8,300 \\
8,370\end{array}$ & ft-lb & $\begin{array}{c}\mathrm{ft}-\mathrm{lb} \\
\\
\end{array}$ & 1. 22 \\
\hline \multicolumn{18}{|c|}{ 38 Inch American National Fine } \\
\hline $\begin{array}{l}1 \\
2 \\
3 \\
5 \\
6\end{array}$ & \begin{tabular}{l|l}
2 \\
3 \\
3 \\
3 \\
3 \\
2 \\
2
\end{tabular} & $\begin{array}{l}0.3193 \\
.3185 \\
.3185 \\
.3201 \\
.3201 \\
.3212\end{array}$ & $\begin{array}{r}+0.0009 \\
+.0001 \\
+.0006 \\
+.0017 \\
+.0017 \\
+.0028\end{array}$ & $\begin{array}{r}0.348 \\
.348 \\
.348 \\
.347 \\
.348 \\
.348\end{array}$ & $\begin{array}{l}0.271 \\
.260 \\
.250 \\
.292 \\
.302 \\
.167\end{array}$ & $\begin{array}{r}0.340 \\
.340 \\
.327 \\
.328 \\
.338 \\
.326\end{array}$ & $\begin{array}{l}0 \\
0 \\
0 \\
0 \\
0 \\
0\end{array}$ & $\begin{array}{r}20 \\
20 \\
0 \\
26 \\
20 \\
39\end{array}$ & $\begin{array}{r}0.0030 \\
.0040 \\
.0037 \\
.0035 \\
.0036 \\
.0033\end{array}$ & $\begin{array}{r}0.15 \\
.15 \\
.16 \\
.15 \\
-. . .\end{array}$ & $\begin{array}{l}134 \\
128 \\
134 \\
128\end{array}$ & 131 & $\begin{array}{r}0.16 \\
.15\end{array}$ & $\begin{array}{r}9,650 \\
10,600\end{array}$ & $\begin{array}{l}117 \\
125\end{array}$ & \} 121 & 1.08 \\
\hline
\end{tabular}


3/8 Inch Dardelet

\begin{tabular}{|c|c|c|c|c|c|c|c|c|c|c|c|c|c|c|c|c|c|}
\hline $\begin{array}{l}1 \\
2 \\
3 \\
5 \\
6\end{array}$ & $\begin{array}{l}3 \\
2 \\
2 \\
2 \\
2 \\
3\end{array}$ & $\begin{array}{l}.3267 \\
.3251 \\
.3267 \\
.3271 \\
.3263 \\
.3260\end{array}$ & $\begin{array}{r}+0.0017 \\
+.0001 \\
+.0017 \\
+.0021 \\
+.0013 \\
+.0010\end{array}$ & $\mid$\begin{tabular}{l} 
\\
\hdashline-1
\end{tabular} & $\begin{array}{r}0.135 \\
.120 \\
.115 \\
.125 \\
.083 \\
\hdashline\end{array}$ & $\begin{array}{r}0.503 \\
.501 \\
.501 \\
.500 \\
.503 \\
.512\end{array}$ & $\begin{array}{l}0 \\
0 \\
0 \\
0 \\
0\end{array}$ & $\begin{array}{l}26 \\
13 \\
13 \\
13 \\
13\end{array}$ & $\begin{array}{r}0.0004 \\
.0028 \\
.0005 \\
.0006 \\
.0006\end{array}$ & $\begin{array}{r}0.16 \\
.14 \\
.16 \\
.15 \\
\end{array}$ & $\begin{array}{l}128 \\
128 \\
134 \\
134 \\
\end{array}$ & 131 & $\begin{array}{l}0.14 \\
.15\end{array}$ & $\begin{array}{r}10,660 \\
9,680\end{array}$ & $\begin{array}{l}122 \\
121\end{array}$ & 122 & 1.08 \\
\hline
\end{tabular}

1/2 Inch American National Coarse

\begin{tabular}{|c|c|c|c|c|c|c|c|c|c|c|c|c|c|c|c|c|c|}
\hline $\begin{array}{l}1 \\
2 \\
3 \\
4 \\
5 \\
6\end{array}$ & $\begin{array}{l}2 \\
2 \\
2 \\
2 \\
2 \\
3 \\
2\end{array}$ & $\begin{array}{r}0.3909 \\
.3909 \\
.3921 \\
.3949 \\
.3913 \\
.3898\end{array}$ & $\begin{array}{r}-0.0054 \\
-.0054 \\
-.0042 \\
-.0014 \\
-.0050 \\
-.0065\end{array}$ & $\begin{array}{r}0.448 \\
.448 \\
.448 \\
.448 \\
.448 \\
.448\end{array}$ & $\begin{array}{r}0.256 \\
.320 \\
.245 \\
.288 \\
.224 \\
.234\end{array}$ & $\begin{array}{r}0.437 \\
.443 \\
.436 \\
.438 \\
.438 \\
.440\end{array}$ & $\begin{array}{l}0 \\
0 \\
0 \\
0 \\
0 \\
0\end{array}$ & $\begin{array}{r}5 \\
24 \\
5 \\
5 \\
10 \\
39\end{array}$ & $\begin{array}{r}0.0070 \\
.0030 \\
.0072 \\
.0073 \\
.0073 \\
.0033\end{array}$ & $\begin{array}{r}0.17 \\
.16 \\
.17 \\
.17 \\
. . .\end{array}$ & $\begin{array}{l}250 \\
236 \\
243 \\
250 \\
-\end{array}$ & 245 & $\begin{array}{r}0.18 \\
.16\end{array}$ & $\begin{array}{l}15,260 \\
16,070\end{array}$ & $\begin{array}{l}216 \\
199\end{array}$ & 208 & 1. 18 \\
\hline
\end{tabular}

1/2 Inch American National Fine

\begin{tabular}{r|r|r|r|r|r|r|r|r|r|}
\hline & 2 & 0.4331 & +0.0007 & 0.466 & 0.192 & 0.437 & 0 & 19 & 0.009 \\
2 & 2 & .4311 & -.0013 & .466 & .309 & .437 & 0 & 24 & \\
3 & .4342 & +.0018 & .466 & .277 & .438 & 0 & 34 \\
5 & 2 & .4323 & -.0001 & .466 & .299 & .437 & 0 & 24 \\
\hline \\
\hline
\end{tabular}

\begin{tabular}{r|r}
0.0023 & 0. \\
.0023 &. \\
.0015 &. \\
.0015 &. \\
.0035 &. \\
.0035 & - \\
\hline
\end{tabular}

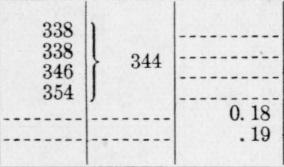

\begin{tabular}{|c|c|c|} 
& & \\
\hdashline 18,190 & 268 & \\
18,870 & 292 & 280 \\
\hline
\end{tabular}

1/2 Inch Dardelet

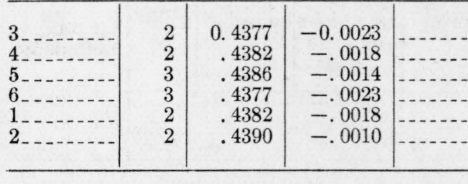

0.0025

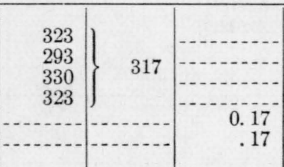

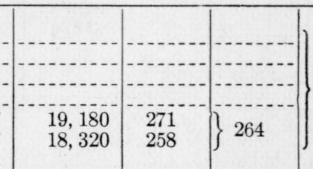

1.20

1 Did not comply with requirements. 
TABLE 7.-Results of measurements and tests: monel metal-Continued

\begin{tabular}{|c|c|c|c|c|c|c|c|c|c|c|c|c|c|c|c|c|c|}
\hline 1 & 2 & 3 & 4 & 5 & 6 & 7 & \multicolumn{2}{|c|}{8} & 9 & 10 & 11 & 12 & 13 & 14 & 15 & 16 & 17 \\
\hline \multicolumn{18}{|c|}{$5 / 8$ Inch American National Coarse } \\
\hline \multirow{3}{*}{$\begin{array}{c}\text { Specimen } \\
\text { no. }\end{array}$} & \multicolumn{5}{|c|}{ Bolt } & \multicolumn{3}{|c|}{ Nut } & & \multicolumn{3}{|c|}{ Impact } & \multicolumn{4}{|c|}{ Static } & \multirow{3}{*}{$\frac{\text { Ratio }}{\text { Impact work (col. 12) }}$} \\
\hline & \multirow[b]{2}{*}{$\begin{array}{l}\text { Bar } \\
\text { no. }\end{array}$} & \multirow[b]{2}{*}{$\begin{array}{c}\text { Actual } \\
\text { minor } \\
\text { diameter }\end{array}$} & \multirow{2}{*}{$\begin{array}{c}\text { Differ- } \\
\text { ence be- } \\
\text { tween ac- } \\
\text { tual and } \\
\text { minimum } \\
\text { minor } \\
\text { diameter }\end{array}$} & \multirow[b]{2}{*}{$\begin{array}{l}\text { Pitch } \\
\text { diam- } \\
\text { eter }\end{array}$} & \multirow{2}{*}{$\begin{array}{l}\text { Angle be- } \\
\text { tween axis } \\
\text { of large } \\
\text { thread and } \\
\text { axis of bolt }\end{array}$} & \multirow[b]{2}{*}{$\begin{array}{l}\text { Thick- } \\
\text { ness }\end{array}$} & \multirow{2}{*}{\multicolumn{2}{|c|}{$\begin{array}{l}\text { Obliquity } \\
\text { of bearing } \\
\text { face }\end{array}$}} & \multirow{2}{*}{$\begin{array}{c}\text { Clearance } \\
\text { between } \\
\text { nut and } \\
\text { bolt }\end{array}$} & \multirow[b]{2}{*}{$\begin{array}{l}\text { Stretch, } \\
5 \text { diam- } \\
\text { eters }\end{array}$} & \multicolumn{2}{|c|}{ Impact work } & \multirow[b]{2}{*}{$\begin{array}{l}\text { Stretch, } \\
5 \text { diam- } \\
\text { eters }\end{array}$} & \multirow[b]{2}{*}{$\begin{array}{l}\text { Maxi- } \\
\text { mum } \\
\text { load }\end{array}$} & \multicolumn{2}{|c|}{ Static work } & \\
\hline & & & & & & & & & & & $\begin{array}{l}\text { Each } \\
\text { speci- } \\
\text { men }\end{array}$ & $\begin{array}{c}\text { A ver- } \\
\text { age }\end{array}$ & & & $\begin{array}{l}\text { Each } \\
\text { speci- } \\
\text { men }\end{array}$ & $\begin{array}{c}\text { Aver- } \\
\text { age }\end{array}$ & \\
\hline $\begin{array}{l}1 \\
2 \\
3 \\
4 \\
5 \\
6\end{array}$ & $\begin{array}{r}4 \\
3 \\
.4 \\
4 \\
3 \\
1\end{array}$ & $\begin{array}{c}\text { in. } \\
0.4941 \\
.4941 \\
.4917 \\
.4921 \\
.4941 \\
.4917\end{array}$ & $\begin{array}{c}\text { in. } \\
-0.0087 \\
-.0087 \\
-.0111 \\
-.0107 \\
-.0087 \\
-.0111\end{array}$ & $\begin{array}{r}\text { in. } \\
10.561 \\
.564 \\
.562 \\
.562 \\
.562 \\
.562\end{array}$ & $\begin{array}{l}\text { deg } \\
0.196 \\
.218 \\
.306 \\
.240 \\
.240 \\
.218\end{array}$ & $\begin{array}{l}\text { in. } \\
0.548 \\
.549 \\
.548 \\
.551 \\
.548 \\
.547\end{array}$ & $\begin{array}{r}\operatorname{deg} \\
0 \\
0 \\
0 \\
0 \\
0 \\
0\end{array}$ & $\begin{array}{r}\min \\
0 \\
15 \\
8 \\
4 \\
8 \\
23\end{array}$ & $\begin{array}{l}\text { in. } \\
0.0068 \\
.0041 \\
.0083 \\
.0073 \\
.0058 \\
.0052\end{array}$ & $\begin{array}{c}\text { in. } \\
0.21 \\
.21 \\
.24 \\
.21\end{array}$ & $\begin{array}{r}\mathrm{ft}-\mathrm{lb} \\
451 \\
443 \\
466 \\
451 \\
-\mathbf{P}^{---}\end{array}$ & $\begin{array}{r}f t-1 b \\
453\end{array}$ & $\begin{array}{l}\text { in. } \\
0.18 \\
.21\end{array}$ & $\begin{array}{l}24,580 \\
23,650\end{array}$ & $\mathrm{ft}-\mathrm{lb}$ & $\begin{array}{c}\mathrm{ft}-\mathrm{lb} \\
374\end{array}$ & 1. 21 \\
\hline \multicolumn{18}{|c|}{ 5/8 Inch American National Fine } \\
\hline $\begin{array}{l}1 \ldots \ldots \\
2 \ldots \ldots \\
3 \ldots \ldots \\
4 \ldots \ldots \\
5 \ldots-\ldots \\
6 \ldots \ldots\end{array}$ & \begin{tabular}{l|l}
2 & \\
4 & \\
1 & \\
4 \\
1 \\
1
\end{tabular} & $\begin{array}{r}0.5476 \\
.5488 \\
.5488 \\
.5480 \\
.5476 \\
.5472\end{array}$ & $\begin{array}{r}-0.0022 \\
-.0010 \\
-.0010 \\
-.0018 \\
-.0022 \\
-.0026\end{array}$ & $\begin{array}{r}0.588 \\
.586 \\
.588 \\
.587 \\
.588 \\
.587\end{array}$ & $\begin{array}{l}0.153 \\
.273 \\
.295 \\
.251 \\
.350 \\
.262\end{array}$ & $\begin{array}{l}0.545 \\
.547 \\
.548 \\
.547 \\
.545 \\
.540\end{array}$ & $\begin{array}{l}0 \\
0 \\
0 \\
0 \\
0 \\
0\end{array}$ & $\begin{array}{l}8 \\
8 \\
4 \\
8 \\
4 \\
8\end{array}$ & $\begin{array}{r}0.0033 \\
.0035 \\
.0044 \\
.0029 \\
.0048 \\
.0038\end{array}$ & $\begin{array}{r}0.26 \\
.27 \\
.29 \\
.28 \\
-. .\end{array}$ & $\begin{array}{l}628 \\
697 \\
711 \\
702\end{array}$ & 684 & $\begin{array}{r}0.25 \\
.26\end{array}$ & $\begin{array}{l}27,690 \\
27,520\end{array}$ & $\begin{array}{l}526 \\
558\end{array}$ & 542 & 1. 26 \\
\hline
\end{tabular}


5/8 Inch Dardelet

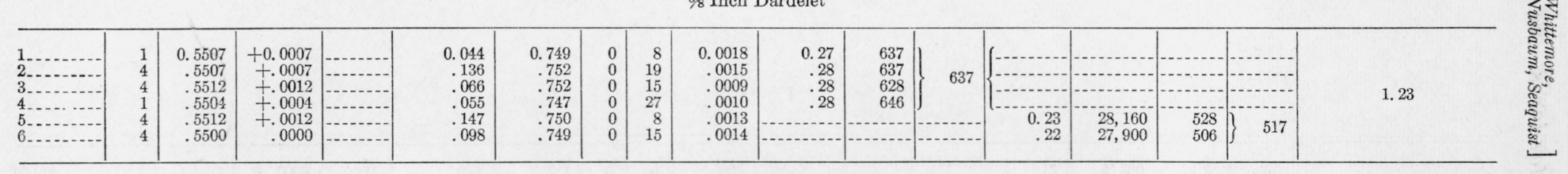

3/4 Inch American National Coarse

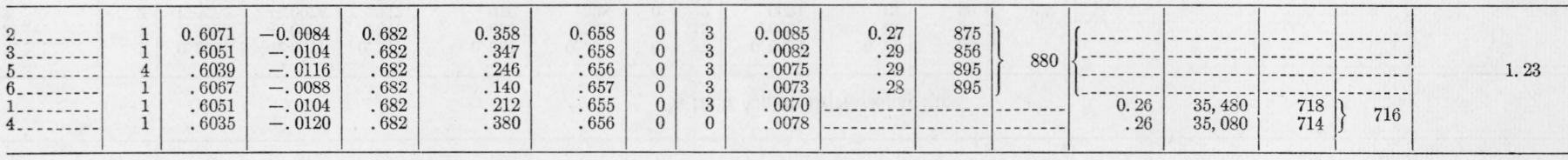

3/4 Inch American National Fine

\begin{tabular}{|c|c|c|c|c|c|c|c|c|c|c|c|c|c|c|c|c|c|}
\hline $\begin{array}{l}1 \\
2 \\
5 \\
6 \\
3 \\
4 \\
4\end{array}$ & \begin{tabular}{r}
1 \\
4 \\
\hdashline 4 \\
1
\end{tabular} & $\begin{array}{l}0.6630 \\
.6618 \\
.6622 \\
.6661 \\
.6634 \\
.6602\end{array}$ & $\begin{array}{r}-0.0026 \\
-.0038 \\
-.0034 \\
+.0005 \\
-.0022 \\
-.0054\end{array}$ & $\begin{array}{l}0.708 \\
.708 \\
.708 \\
.708 \\
.708 \\
.708\end{array}$ & $\begin{array}{r}0.391 \\
.324 \\
.402 \\
.212 \\
.134 \\
.414\end{array}$ & $\begin{array}{l}0.659 \\
.657 \\
.659 \\
.657 \\
.658 \\
.653\end{array}$ & $\begin{array}{l}0 \\
0 \\
0 \\
0 \\
0 \\
0\end{array}$ & $\begin{array}{l}3 \\
3 \\
3 \\
3 \\
3 \\
0\end{array}$ & $\begin{array}{l}0.0034 \\
.0037 \\
.0022 \\
.0029 \\
.0020 \\
.0019\end{array}$ & $\begin{array}{r}0.35 \\
.35 \\
.34 \\
.34 \\
\end{array}$ & $\begin{array}{l}1,250 \\
1,288 \\
1,231 \\
1,280 \\
-\cdots \cdot-.\end{array}$ & $\left\{\begin{array}{l}1,262 \\
\end{array}\right.$ & $\begin{array}{r}0.31 \\
.31\end{array}$ & $\begin{array}{l}40,330 \\
40,270\end{array}$ & $\begin{array}{l}977 \\
976\end{array}$ & 976 & 1. 29 \\
\hline
\end{tabular}

3/4 Inch Dardelet

\begin{tabular}{|c|c|c|c|c|c|c|c|c|c|c|c|c|c|c|c|c|c|}
\hline $\begin{array}{l}1 \\
2 \\
3 \\
4 \\
5 \\
6\end{array}$ & \begin{tabular}{l}
1 \\
\hdashline 1 \\
1 \\
1 \\
1
\end{tabular} & $\begin{array}{r}0.6762 \\
.6728 \\
.6756 \\
.6756 \\
.6752 \\
.6756\end{array}$ & $\begin{array}{r}+0.0012 \\
-.0022 \\
+.0006 \\
+.0006 \\
+.0002 \\
+.0006\end{array}$ & 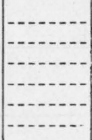 & $\begin{array}{l}0.101 \\
.151 \\
.196 \\
.095 \\
.151 \\
.123\end{array}$ & $\begin{array}{l}0.878 \\
.877 \\
.875 \\
.865 \\
.869 \\
.880\end{array}$ & $\begin{array}{l}0 \\
0 \\
0 \\
0 \\
0 \\
0\end{array}$ & $\begin{array}{l}13 \\
10 \\
20 \\
10 \\
13 \\
10\end{array}$ & $\begin{array}{l}0.0011 \\
.0014 \\
.0006 \\
.0027 \\
.0026 \\
.0019\end{array}$ & $\begin{array}{r}0.34 \\
.36 \\
.35 \\
.35 \\
\\
\hdashline\end{array}$ & $\begin{array}{l}1,211 \\
1,240 \\
1,221 \\
1,211\end{array}$ & 1,221 & $\begin{array}{r}0.33 \\
.32\end{array}$ & $\begin{array}{l}41,120 \\
41,450\end{array}$ & $\begin{array}{l}1,060 \\
1,030\end{array}$ & 1,045 & 1. 17 \\
\hline
\end{tabular}

${ }^{1}$ Did not comply with requirements. 
TABLE 8.-Results of measurements and tests: bronze

\begin{tabular}{|c|c|c|c|c|c|c|c|c|c|c|c|c|c|c|c|c|}
\hline 1 & 2 & 3 & 4 & 5 & 6 & 7 & 8 & 9 & 10 & 11 & 12 & 13 & 14 & 15 & 16 & 17 \\
\hline
\end{tabular}

3/\& Inch American National Coarse

\begin{tabular}{|c|c|c|c|c|c|c|c|c|c|c|c|c|c|c|c|c|c|}
\hline \multirow{3}{*}{$\begin{array}{c}\text { Specimen } \\
\text { no. }\end{array}$} & \multicolumn{5}{|c|}{ Bolt } & \multicolumn{3}{|c|}{ Nut } & \multirow{3}{*}{$\begin{array}{c}\text { Clearance } \\
\text { between } \\
\text { nut and } \\
\text { bolt }\end{array}$} & \multicolumn{3}{|c|}{ Impact } & \multicolumn{4}{|c|}{ Static } & \multirow{3}{*}{$\begin{array}{c}\text { Ratio } \\
\text { Impact work (col. 12) } \\
\text { Static work (col. 16) }\end{array}$} \\
\hline & \multirow[b]{2}{*}{$\begin{array}{l}\text { Bar } \\
\text { no. }\end{array}$} & \multirow[b]{2}{*}{$\begin{array}{c}\text { Actual } \\
\text { minor } \\
\text { diameter }\end{array}$} & \multirow{2}{*}{$\begin{array}{c}\text { Differ- } \\
\text { ence be- } \\
\text { tween ac- } \\
\text { tual and } \\
\text { minimum } \\
\text { minor } \\
\text { diameter }\end{array}$} & \multirow[b]{2}{*}{$\begin{array}{l}\text { Pitch } \\
\text { diam- } \\
\text { eter }\end{array}$} & \multirow{2}{*}{$\begin{array}{l}\text { Angle be- } \\
\text { tween axis } \\
\text { of large } \\
\text { thread and } \\
\text { axis of bolt }\end{array}$} & \multirow[b]{2}{*}{$\begin{array}{l}\text { Thick- } \\
\text { ness }\end{array}$} & \multirow{2}{*}{\multicolumn{2}{|c|}{$\begin{array}{c}\text { Obliquity } \\
\text { of bearing } \\
\text { face }\end{array}$}} & & \multirow[b]{2}{*}{$\begin{array}{l}\text { Stretch, } \\
5 \text { diam- } \\
\text { eters }\end{array}$} & \multicolumn{2}{|c|}{ Impact work } & \multirow[b]{2}{*}{$\begin{array}{l}\text { Stretch, } \\
5 \text { diam- } \\
\text { eters }\end{array}$} & \multirow[b]{2}{*}{$\begin{array}{l}\text { Maxi- } \\
\text { mum } \\
\text { load }\end{array}$} & \multicolumn{2}{|c|}{ Static work } & \\
\hline & & & & & & & & & & & $\begin{array}{l}\text { Each } \\
\text { speci- } \\
\text { men }\end{array}$ & $\begin{array}{l}\text { A ver- } \\
\text { age }\end{array}$ & & & $\begin{array}{l}\text { Each } \\
\text { speci- } \\
\text { men }\end{array}$ & $\begin{array}{l}\text { Aver- } \\
\text { age }\end{array}$ & \\
\hline $\begin{array}{l}1 \\
2 \\
4 \\
5 \\
3 \\
3 \\
6\end{array}$ & $\begin{array}{l}1 \\
1 \\
2 \\
2 \\
2 \\
2\end{array}$ & $\begin{array}{l}\text { in. } \\
0.2866 \\
.2870 \\
.2862 \\
.2870 \\
.2862 \\
.2838\end{array}$ & $\begin{array}{c}\text { in. } \\
-0.0039 \\
-.0035 \\
-.0043 \\
-.0035 \\
-.0043 \\
-.0067\end{array}$ & $\begin{array}{c}\text { in. } \\
0.332 \\
.331 \\
1.330 \\
.331 \\
.331 \\
1.329\end{array}$ & $\begin{array}{c}\mathrm{deg} \\
0.031 \\
.036 \\
.031 \\
.068 \\
.083 \\
.047\end{array}$ & $\begin{array}{l}\text { in. } \\
0.325 \\
.321 \\
.322 \\
.325 \\
.329 \\
.326\end{array}$ & $\begin{array}{r}\operatorname{deg} \\
0 \\
0 \\
0 \\
0 \\
0 \\
0\end{array}$ & $\begin{array}{r}\min \\
7 \\
7 \\
7 \\
7 \\
7 \\
7\end{array}$ & $\begin{array}{l}\text { in. } \\
0.0039 \\
.0057 \\
.0053 \\
.0048 \\
.0045 \\
.0056\end{array}$ & $\begin{array}{r}\text { in. } \\
0.13 \\
.12 \\
.10 \\
.08\end{array}$ & $\begin{array}{r}\mathrm{ft}-\mathrm{lb} \\
70 \\
50 \\
70 \\
60\end{array}$ & ft-lb & $\begin{array}{r}0.10 \\
.07\end{array}$ & $\begin{array}{l}7,230 \\
6,930\end{array}$ & $\mathrm{ft}-\mathrm{lb}$ & ft-lb & 1. 22 \\
\hline
\end{tabular}

3/8 Inch American National Fine

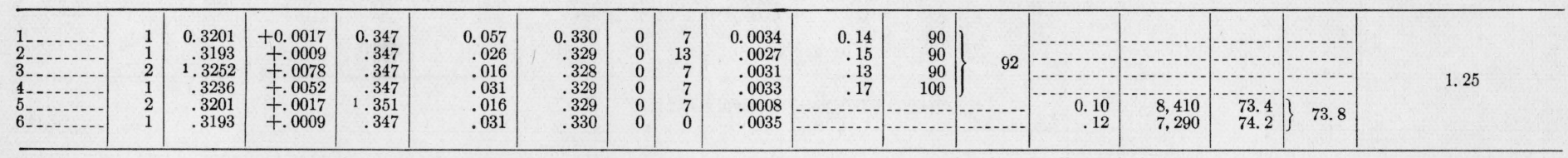


s/8 Inch Dardelet

\begin{tabular}{|c|c|c|c|c|c|c|c|c|c|c|c|c|c|c|c|c|c|}
\hline $\begin{array}{l}1 \\
2 \\
3 \\
4 \\
5 \\
6\end{array}$ & $\begin{array}{l}1 \\
1 \\
1 \\
2 \\
2 \\
3\end{array}$ & $\begin{array}{r}0.3260 \\
.3263 \\
.3260 \\
.3248 \\
.3263 \\
.3248\end{array}$ & $\begin{array}{r}+0.0010 \\
+.0013 \\
+.0010 \\
+.0002 \\
+.0013 \\
-.0002\end{array}$ & $\mid \begin{array}{l} \\
\end{array}$ & $\begin{array}{r}0.182 \\
.083 \\
.182 \\
.156 \\
.172 \\
.130\end{array}$ & $\begin{array}{r}0.503 \\
.500 \\
.502 \\
.501 \\
.501 \\
.501\end{array}$ & $\begin{array}{l}0 \\
0 \\
0 \\
0 \\
0 \\
0\end{array}$ & \begin{tabular}{l|}
46 \\
13 \\
59 \\
33 \\
33 \\
20
\end{tabular} & $\begin{array}{r}0.0007 \\
.0008 \\
.0009 \\
.0019 \\
.0012 \\
.0021\end{array}$ & $\begin{array}{r}0.18 \\
.14 \\
.18 \\
.12 \\
\\
\end{array}$ & $\begin{array}{r}100 \\
95 \\
100 \\
95 \\
-.-\end{array}$ & $\begin{array}{r}\}^{9} \\
\end{array}$ & $\begin{array}{r}0.11 \\
.10\end{array}$ & $\begin{array}{l}8,430 \\
8,310\end{array}$ & $\begin{array}{c}\ldots . . \\
82.6 \\
74.2\end{array}$ & $\begin{array}{c}\ldots .1 \\
78.4\end{array}$ & 1. 25 \\
\hline \multicolumn{18}{|c|}{ 1/2 Inch American National Coarse } \\
\hline $\begin{array}{l}1 \\
2 \\
3 \\
4 \\
5 \\
6\end{array}$ & $\begin{array}{r}1 \\
4 \\
2 \\
4 \\
-2 \\
1\end{array}$ & $\begin{array}{r}0.3906 \\
.3909 \\
.3957 \\
.3886 \\
.3921 \\
.3886\end{array}$ & $\begin{array}{r}-0.0057 \\
-.0054 \\
-.0006 \\
-.0077 \\
-.0042 \\
-.0077\end{array}$ & $\begin{array}{l}0.447 \\
.446 \\
.447 \\
.446 \\
.446 \\
.446\end{array}$ & $\begin{array}{l}0.000 \\
.021 \\
.000 \\
.000 \\
.000 \\
.021\end{array}$ & $\begin{array}{r}0.433 \\
.433 \\
.435 \\
.437 \\
.438 \\
.435\end{array}$ & $\begin{array}{l}0 \\
0 \\
0 \\
0 \\
0 \\
0\end{array}$ & $\begin{array}{l}5 \\
5 \\
5 \\
5 \\
0 \\
5\end{array}$ & $\begin{array}{r}0.0049 \\
.0046 \\
.0045 \\
.0057 \\
.0053 \\
.0054\end{array}$ & $\begin{array}{r}0.17 \\
.19 \\
.16 \\
.17 \\
\end{array}$ & $\begin{array}{l}177 \\
183 \\
177 \\
170\end{array}$ & 177 & $\begin{array}{r}0.13 \\
.14\end{array}$ & $\begin{array}{l}12,720 \\
11,360\end{array}$ & $\begin{array}{l}136 \\
131\end{array}$ & 134 & 1. 32 \\
\hline \multicolumn{18}{|c|}{$1 / 2$ Inch American National Fine } \\
\hline $\begin{array}{l}1 \\
2 \\
3 \\
4 \\
5 \\
6\end{array}$ & $\begin{array}{l}1 \\
1 \\
4 \\
2 \\
2 \\
4\end{array}$ & $\begin{array}{r}0.4280 \\
.4272 \\
.4291 \\
.4291 \\
.4291 \\
.4295\end{array}$ & $\begin{array}{r}-0.0044 \\
-.0052 \\
-.0033 \\
-.0033 \\
-.0033 \\
-.0029\end{array}$ & $\begin{array}{r}10.464 \\
.466 \\
.466 \\
.466 \\
.466 \\
.466\end{array}$ & $\begin{array}{l}0.000 \\
.000 \\
.021 \\
.000 \\
.021 \\
.021\end{array}$ & $\begin{array}{l}0.435 \\
.437 \\
.435 \\
.437 \\
.436 \\
.437\end{array}$ & $\begin{array}{l}0 \\
0 \\
0 \\
0 \\
0 \\
0\end{array}$ & $\begin{array}{l}5 \\
5 \\
0 \\
5 \\
5 \\
0\end{array}$ & $\begin{array}{r}0.0020 \\
.0019 \\
.0020 \\
.0022 \\
.0027 \\
.0020\end{array}$ & \begin{tabular}{r|}
0.24 \\
.24 \\
.21 \\
.18 \\
\hdashline... \\
...
\end{tabular} & \begin{tabular}{c}
250 \\
250 \\
236 \\
229 \\
\hdashline$\ldots .$. \\
\end{tabular} & \}$^{2} 241$ & $\begin{array}{r}0.12 \\
.18\end{array}$ & $\begin{array}{l}14,460 \\
13.410\end{array}$ & $\begin{array}{l}148 \\
203\end{array}$ & 176 & 1. 37 \\
\hline \multicolumn{18}{|c|}{$1 / 2$ Inch Dardelet } \\
\hline $\begin{array}{l}3 \ldots \\
45 \\
5 \\
6 \\
1 \\
2\end{array}$ & $\begin{array}{l}2 \\
2 \\
3 \\
1 \\
3 \\
1\end{array}$ & $\begin{array}{l}0.4390 \\
.4394 \\
.4394 \\
.4380 \\
.4394 \\
.4390\end{array}$ & $\begin{array}{r}-0.0010 \\
-.0006 \\
-.0006 \\
-.0020 \\
-.0006 \\
-.0010\end{array}$ & 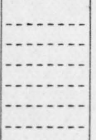 & $\begin{array}{r}0.133 \\
.128 \\
.112 \\
.128 \\
.133 \\
.117\end{array}$ & \begin{tabular}{r|}
0.627 \\
.626 \\
.626 \\
.627 \\
.623 \\
.624
\end{tabular} & $\begin{array}{l}0 \\
0 \\
0 \\
0 \\
0 \\
0\end{array}$ & $\begin{array}{l}29 \\
14 \\
14 \\
14 \\
10 \\
10\end{array}$ & $\begin{array}{r}0.0012 \\
.0010 \\
.0010 \\
.0018 \\
.0021 \\
.0017\end{array}$ & $\begin{array}{c}0.21 \\
.22 \\
.20 \\
.24 \\
. . .\end{array}$ & $\begin{array}{l}264 \\
264 \\
243 \\
257\end{array}$ & 257 & $\begin{array}{r}0.18 \\
.20\end{array}$ & $\begin{array}{l}15,240 \\
13,350\end{array}$ & 230 & 228 & 1. 13 \\
\hline
\end{tabular}

1 Did not comply with requirements. 
TABLE 8.-Results of measurements and tests: bronze-Continued

\begin{tabular}{l|l|l|l|l|l|l|l|l|l|l|l|l|l|l|l|l|l|l|l|l|}
\hline 1 & 2 & 3 & 4 & 5 & 6 & 7 & 8 & 9 & 10 & 11 & 12 & 13 \\
\hline
\end{tabular}

5/8 Inch American National Coarse

\begin{tabular}{|c|c|c|c|c|c|c|c|c|c|c|c|c|c|c|c|c|c|}
\hline \multirow{3}{*}{$\begin{array}{l}\text { Specimen } \\
\text { no. }\end{array}$} & \multicolumn{5}{|c|}{ Bolt } & \multicolumn{3}{|c|}{ Nut } & \multirow{3}{*}{$\begin{array}{c}\text { Clearance } \\
\text { between } \\
\text { nut and } \\
\text { bolt }\end{array}$} & \multicolumn{3}{|c|}{ Impact } & \multicolumn{4}{|c|}{ Static } & \multirow{3}{*}{$\begin{array}{l}\text { Ratio } \\
\text { Impact work (col. 12) } \\
\text { Static work (col. 16) }\end{array}$} \\
\hline & \multirow[b]{2}{*}{$\begin{array}{l}\text { Bar } \\
\text { no. }\end{array}$} & \multirow[b]{2}{*}{$\begin{array}{l}\text { Actual } \\
\text { minor } \\
\text { diameter }\end{array}$} & \multirow{2}{*}{$\begin{array}{c}\text { Differ- } \\
\text { ence be- } \\
\text { tween ac- } \\
\text { tual and } \\
\text { minimum } \\
\text { minor } \\
\text { diameter }\end{array}$} & \multirow[b]{2}{*}{$\begin{array}{l}\text { Pitch } \\
\text { diam- } \\
\text { eter }\end{array}$} & \multirow{2}{*}{$\begin{array}{c}\text { Angle be- } \\
\text { tween axis } \\
\text { of large } \\
\text { thread and } \\
\text { axis of bolt }\end{array}$} & \multirow[b]{2}{*}{$\begin{array}{l}\text { Thick- } \\
\text { ness }\end{array}$} & \multirow{2}{*}{\multicolumn{2}{|c|}{$\begin{array}{c}\text { Obliquity } \\
\text { of bearing } \\
\text { face }\end{array}$}} & & \multirow[b]{2}{*}{$\begin{array}{l}\text { Stretch, } \\
5 \text { diam- } \\
\text { eters }\end{array}$} & \multicolumn{2}{|c|}{ Impact work } & \multirow[b]{2}{*}{$\begin{array}{l}\text { Stretch, } \\
5 \text { diam- } \\
\text { eters }\end{array}$} & \multirow[b]{2}{*}{$\begin{array}{l}\text { Maxi- } \\
\text { mum } \\
\text { load }\end{array}$} & \multicolumn{2}{|c|}{ Static work } & \\
\hline & & & & & & & & & & & $\begin{array}{l}\text { Each } \\
\text { speci- } \\
\text { men }\end{array}$ & $\begin{array}{l}\text { A ver- } \\
\text { age }\end{array}$ & & & $\begin{array}{l}\text { Each } \\
\text { speci- } \\
\text { men }\end{array}$ & $\begin{array}{l}\text { Aver- } \\
\text { age }\end{array}$ & \\
\hline $\begin{array}{l}2 \\
3 \\
4 \\
6 \\
1 \\
5\end{array}$ & $\begin{array}{l}5 \\
3 \\
5 \\
4 \\
5 \\
4 \\
4\end{array}$ & $\begin{array}{c}\text { in. } \\
0.4949 \\
.5028 \\
.4933 \\
.5012 \\
.5035 \\
.5028\end{array}$ & $\begin{array}{r}\text { in. } \\
-0.0079 \\
.0000 \\
-.0095 \\
-.0016 \\
+.0007 \\
.0000\end{array}$ & $\begin{array}{l}\text { in. } \\
0.562 \\
1.561 \\
1.561 \\
1.560 \\
1.561 \\
.562\end{array}$ & $\begin{array}{r}\operatorname{deg} \\
0.000 \\
.000 \\
.000 \\
.000 \\
.000 \\
.022\end{array}$ & $\begin{array}{l}\text { in. } \\
0.548 \\
.539 \\
.547 \\
.549 \\
.546 \\
.545\end{array}$ & $\begin{array}{r}\operatorname{deg} \\
0 \\
0 \\
0 \\
0 \\
0 \\
0\end{array}$ & $\begin{array}{r}\min \\
4 \\
4 \\
0 \\
8 \\
4 \\
11\end{array}$ & $\begin{array}{l}\text { in. } \\
0.0045 \\
.0055 \\
.0052 \\
.0062 \\
.0042 \\
.0048\end{array}$ & $\begin{array}{l}\text { in. } \\
0.11 \\
.16 \\
.14 \\
.22\end{array}$ & $\begin{array}{r}\text { ft-lb } \\
250 \\
346 \\
250 \\
362\end{array}$ & $\begin{array}{r}\text { ft-ib } \\
302\end{array}$ & $\begin{array}{r}0.08 \\
.16\end{array}$ & $\begin{array}{l}23,200 \\
19,170\end{array}$ & ft-1b & ft $-\mathrm{lb}$ & 1.37 \\
\hline
\end{tabular}

$5 / 8$ Inch American National Fine

\begin{tabular}{|c|c|c|c|c|c|c|c|c|c|c|c|c|c|c|c|c|c|}
\hline $\begin{array}{l}1 \\
2 \\
3 \\
4 \\
5 \\
6\end{array}$ & $\begin{array}{l}3 \\
1 \\
4 \\
3 \\
5 \\
4\end{array}$ & $\begin{array}{r}0.5555 \\
.5551 \\
.5551 \\
.5551 \\
.5551 \\
.5567\end{array}$ & $\begin{array}{r}+0.0057 \\
+.0053 \\
+.0053 \\
+.0053 \\
+.0053 \\
+.0069\end{array}$ & $\begin{array}{l}0.587 \\
.587 \\
.587 \\
.587 \\
.587 \\
.586\end{array}$ & $\begin{array}{r}0.000 \\
.000 \\
.000 \\
.038 \\
.000 \\
.000\end{array}$ & $\begin{array}{l}0.544 \\
.551 \\
.547 \\
.545 \\
.545 \\
.545\end{array}$ & $\begin{array}{l}0 \\
0 \\
0 \\
0 \\
0 \\
0\end{array}$ & $\begin{array}{r}8 \\
4 \\
8 \\
11 \\
4 \\
27\end{array}$ & $\begin{array}{r}0.0039 \\
.0039 \\
.0039 \\
.0040 \\
.0045 \\
.0055\end{array}$ & $\begin{array}{r}0.21 \\
.31 \\
.31 \\
.29\end{array}$ & $\begin{array}{l}443 \\
555 \\
555 \\
485\end{array}$ & 510 & $\begin{array}{r}0.14 \\
.29\end{array}$ & $\begin{array}{l}26,740 \\
21,650\end{array}$ & $\begin{array}{l}331 \\
512\end{array}$ & 422 & 1. 21 \\
\hline
\end{tabular}


$5 / 8$ Inch Dardelet

\begin{tabular}{|c|c|c|c|c|c|c|c|c|c|c|c|c|c|c|c|c|}
\hline $\begin{array}{l}1 \\
2 \\
3 \\
4 \\
5 \\
6\end{array}$ & $\begin{array}{l}5 \\
3 \\
3 \\
3 \\
4 \\
5\end{array}$ & $\begin{array}{r}0.5504 \\
.5520 \\
.5507 \\
.5512 \\
.5504 \\
.5507\end{array}$ & $\begin{array}{r}+0.0004 \\
+.0020 \\
+.0007 \\
+.0012 \\
+.0004 \\
+.0007\end{array}$ & $\begin{array}{l}0.147 \\
.115 \\
.093 \\
.126 \\
.071 \\
.104\end{array}$ & $\begin{array}{r}0.752 \\
.751 \\
.749 \\
.752 \\
.752 \\
.750\end{array}$ & $\begin{array}{l}0 \\
0 \\
0 \\
0 \\
0 \\
0\end{array}$ & $\begin{array}{r}0 \\
19 \\
15 \\
11 \\
4 \\
15\end{array}$ & $\begin{array}{l}0.0026 \\
.0013 \\
.0024 \\
.0009 \\
.0027 \\
.0015\end{array}$ & $\begin{array}{r}0.18 \\
.22 \\
.20 \\
.28\end{array}$ & $\begin{array}{l}418 \\
443 \\
434 \\
485\end{array}$ & 445 & $\begin{array}{r}0.23 \\
.12\end{array}$ & $\begin{array}{l}21,350 \\
25,940\end{array}$ & $\begin{array}{l}463 \\
290\end{array}$ & 376 & 1. 18 \\
\hline
\end{tabular}

3/4 Inch American National Coarse

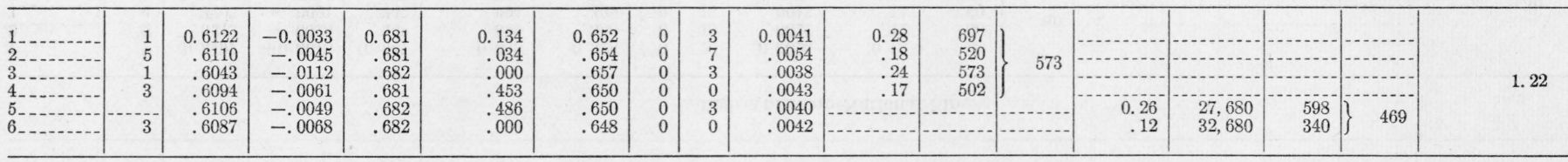

3/4 Inch American National Fine

\begin{tabular}{|c|c|c|c|c|c|c|c|c|c|c|c|c|c|c|c|c|c|}
\hline $\begin{array}{l}2- \\
3- \\
4- \\
5- \\
1- \\
6-\end{array}$ & \begin{tabular}{c}
5 \\
\hdashline.- \\
5 \\
3 \\
4
\end{tabular} & $\begin{array}{l}0.6701 \\
.6693 \\
.6693 \\
.6697 \\
.6701 \\
.6701\end{array}$ & $\begin{array}{r}+0.0045 \\
+.00037 \\
+.0037 \\
+.0041 \\
+.0045 \\
+.0045\end{array}$ & $\begin{array}{l}0.707 \\
.707 \\
.707 \\
.706 \\
.707 \\
.707\end{array}$ & $\begin{array}{r}0.039 \\
.078 \\
.000 \\
.179 \\
.000 \\
.268\end{array}$ & $\begin{array}{r}0.654 \\
.656 \\
.648 \\
.650 \\
.655 \\
.648\end{array}$ & $\begin{array}{l}0 \\
0 \\
0 \\
0 \\
0 \\
0\end{array}$ & $\begin{array}{l}3 \\
3 \\
0 \\
3 \\
0 \\
3\end{array}$ & $\begin{array}{l}0.0038 \\
.0031 \\
.0045 \\
.0055 \\
.0020 \\
.0037\end{array}$ & $\begin{array}{r}0.25 \\
.25 \\
.30 \\
.23\end{array}$ & $\begin{array}{l}826 \\
856 \\
866 \\
788\end{array}$ & 834 & $\begin{array}{r}0.16 \\
.36\end{array}$ & $\begin{array}{l}37,800 \\
31,450\end{array}$ & $\begin{array}{l}530 \\
892\end{array}$ & 711 & 1. 17 \\
\hline
\end{tabular}

$3 / 4$ Inch Dardelet

\begin{tabular}{|c|c|c|c|c|c|c|c|c|c|c|c|c|c|c|c|c|c|}
\hline $\begin{array}{l}1 \\
2 \\
3 \\
4 \\
5 \\
6\end{array}$ & $\begin{array}{l}4 \\
4 \\
3 \\
4 \\
5 \\
5\end{array}$ & $\begin{array}{r}0.6764 \\
.6750 \\
.6756 \\
.6768 \\
.6764 \\
.6768\end{array}$ & $\begin{array}{r}+0.0014 \\
.0000 \\
+.0006 \\
+.0018 \\
+.0014 \\
+.0018\end{array}$ & 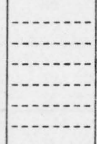 & $\begin{array}{r}0.173 \\
.151 \\
.168 \\
.129 \\
.123 \\
.173\end{array}$ & $\begin{array}{l}0.878 \\
.872 \\
.878 \\
.880 \\
.871 \\
.870\end{array}$ & $\begin{array}{l}0 \\
0 \\
0 \\
0 \\
0 \\
0\end{array}$ & $\begin{array}{r}17 \\
7 \\
10 \\
3 \\
7 \\
13\end{array}$ & $\begin{array}{l}0.0015 \\
.0017 \\
.0014 \\
.0007 \\
.0006 \\
.0009\end{array}$ & $\begin{array}{r}0.32 \\
.34 \\
.20 \\
.30 \\
-.\end{array}$ & $\begin{array}{l}954 \\
944 \\
711 \\
885\end{array}$ & 874 & $\begin{array}{r}0.27 \\
.15\end{array}$ & $\begin{array}{l}38,410 \\
38,590\end{array}$ & $\begin{array}{l}863 \\
511\end{array}$ & 687 & 1. 27 \\
\hline
\end{tabular}

1 Did not comply with requirements. 
TABLE 9.-Results of measurements and tests: brass

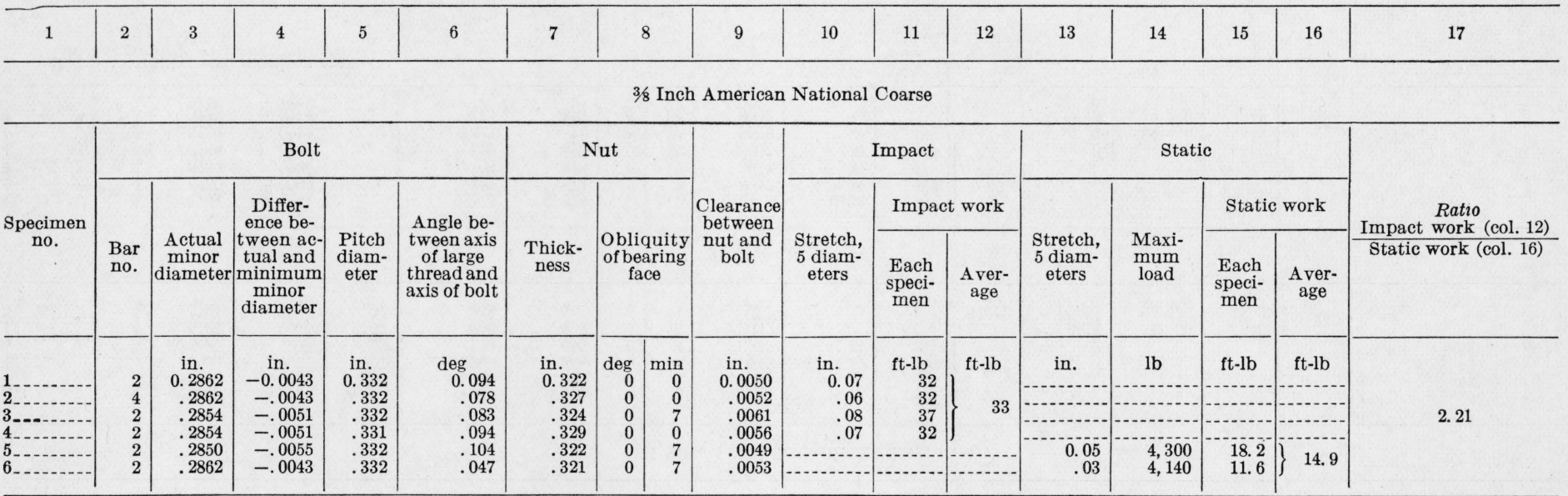

3/8 Inch American National Fine

\begin{tabular}{|c|c|c|c|c|c|c|c|c|c|c|c|c|c|c|c|c|c|}
\hline $\begin{array}{l}1 \\
2 \\
3 \\
4 \\
5 \\
6\end{array}$ & $\begin{array}{l}2 \\
2 \\
4 \\
2 \\
2 \\
4\end{array}$ & $\begin{array}{r}0.3197 \\
.3212 \\
.3205 \\
.3216 \\
.3216 \\
.3181\end{array}$ & $\begin{array}{r}+0.0013 \\
+.0028 \\
+.0021 \\
+.0032 \\
+.0032 \\
-.0003\end{array}$ & $\begin{array}{r}0.347 \\
.348 \\
.347 \\
.347 \\
.347 \\
.346\end{array}$ & $\begin{array}{r}0.089 \\
.099 \\
.094 \\
.063 \\
.089 \\
.073\end{array}$ & $\begin{array}{l}0.328 \\
.330 \\
.329 \\
.328 \\
.330 \\
.328\end{array}$ & $\begin{array}{l}0 \\
0 \\
0 \\
0 \\
0 \\
0\end{array}$ & $\begin{array}{r}26 \\
13 \\
13 \\
7 \\
33 \\
26\end{array}$ & $\begin{array}{l}0.0023 \\
.0021 \\
.0013 \\
.0030 \\
.0010 \\
.0027\end{array}$ & $\begin{array}{r}0.13 \\
.12 \\
.11 \\
.11\end{array}$ & $\begin{array}{l}60 \\
60 \\
60 \\
60 \\
\end{array}$ & 60 & $\begin{array}{r}0.08 \\
.04\end{array}$ & $\begin{array}{l}5,080 \\
5,480\end{array}$ & $\begin{array}{l}35.8 \\
18.4\end{array}$ & 27.1 & 2. 21 \\
\hline
\end{tabular}


36 Inch Dardelet

\begin{tabular}{|c|c|c|c|c|c|c|c|c|c|c|c|c|c|c|c|c|c|}
\hline $\begin{array}{l}1 \\
2 \\
3 \\
4 \\
5 \\
6\end{array}$ & $\begin{array}{l}2 \\
2 \\
2 \\
2 \\
2 \\
2\end{array}$ & $\begin{array}{l}0.3267 \\
.3263 \\
.3263 \\
.3260 \\
.3263 \\
.3267\end{array}$ & $\begin{array}{r}+0.0017 \\
+.0013 \\
+.0013 \\
+.0010 \\
+.0013 \\
+.0017\end{array}$ & 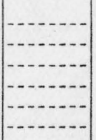 & $\begin{array}{l}0.177 \\
.156 \\
.250 \\
.198 \\
.172 \\
.162\end{array}$ & $\begin{array}{l}0.499 \\
.499 \\
.503 \\
.498 \\
.498 \\
.502\end{array}$ & $\begin{array}{l}0 \\
0 \\
0 \\
0 \\
0 \\
0\end{array}$ & $\begin{array}{l}46 \\
20 \\
33 \\
20 \\
20 \\
33\end{array}$ & $\begin{array}{l}0.0008 \\
.0009 \\
.0005 \\
.0009 \\
.0010 \\
.0010\end{array}$ & $\begin{array}{r}0.15 \\
.14 \\
.15 \\
.14 \\
\end{array}$ & $\begin{array}{l}64 \\
64 \\
70 \\
64\end{array}$ & 66 & $\begin{array}{r}0.09 \\
.08\end{array}$ & $\begin{array}{l}5,150 \\
5,240\end{array}$ & $\begin{array}{l}41.7 \\
36.8\end{array}$ & $\begin{array}{c}- \\
\cdots \\
\cdots\end{array}$ & 1.68 \\
\hline
\end{tabular}

1/2 Inch American National Coarse

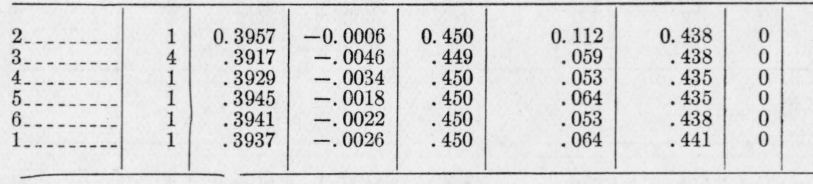

1/2 Inch American National Fine

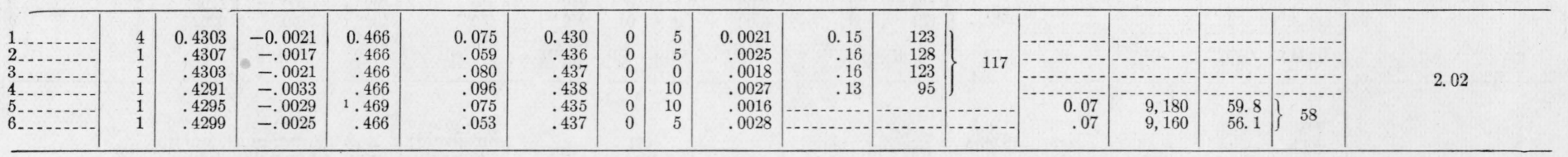

1/2 Inch Dardelet

\begin{tabular}{|c|c|c|c|c|c|c|c|c|c|c|c|c|c|c|c|c|c|}
\hline 3 & $\begin{array}{l}1 \\
1 \\
4 \\
1 \\
1 \\
4\end{array}$ & $\begin{array}{r}0.4386 \\
.4390 \\
.4386 \\
.4394 \\
.4386 \\
.4390\end{array}$ & $\begin{array}{r}-0.0014 \\
-.0010 \\
-.0014 \\
-.0006 \\
-.0014 \\
-.0010\end{array}$ & 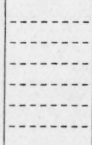 & $\begin{array}{l}0.107 \\
.101 \\
.123 \\
.320 \\
.181 \\
.133\end{array}$ & $\begin{array}{l}0.628 \\
.625 \\
.625 \\
.625 \\
.628 \\
.627\end{array}$ & $\begin{array}{l}0 \\
0 \\
0 \\
0 \\
0 \\
0\end{array}$ & $\begin{array}{l}19 \\
10 \\
19 \\
24 \\
19 \\
19\end{array}$ & $\begin{array}{r}0.0028 \\
.0014 \\
.0014 \\
.0008 \\
.0026 \\
.0029\end{array}$ & $\begin{array}{r}0.16 \\
.18 \\
.15 \\
.19 \\
\end{array}$ & $\begin{array}{l}134 \\
140 \\
128 \\
140\end{array}$ & 136 & $\begin{array}{r}0.09 \\
.07\end{array}$ & $\begin{array}{r}9,570 \\
10,190\end{array}$ & $\begin{array}{l}77.5 \\
70.5\end{array}$ & 74 & 1.84 \\
\hline
\end{tabular}

${ }_{1}$ Did not comply with requirements. 
TABLE 9.-Results of measurements and tests: brass-Continued 


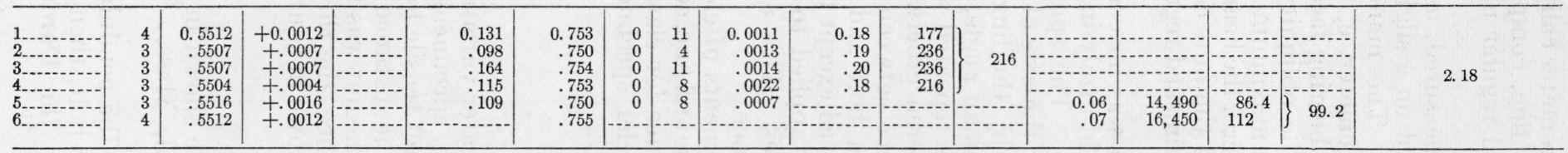

3/4 Inch American National Coarse

\begin{tabular}{|c|c|c|c|c|c|c|c|c|c|c|c|c|c|c|c|c|c|}
\hline $\begin{array}{l}1 \\
2 \\
3 \\
4 \\
5 \\
6\end{array}$ & $\begin{array}{l}5 \\
5 \\
5 \\
5 \\
5 \\
5\end{array}$ & $\begin{array}{r}0.6079 \\
.6130 \\
.6154 \\
.6157 \\
.6094 \\
.6083\end{array}$ & $\begin{array}{r}-0.0076 \\
-.0025 \\
-.0001 \\
+.0002 \\
-.0061 \\
-.0072\end{array}$ & $\begin{array}{r}0.681 \\
1.686 \\
.682 \\
.684 \\
.682 \\
.682\end{array}$ & $\begin{array}{r}0.022 \\
.000 \\
.084 \\
.000 \\
.000 \\
.134\end{array}$ & $\begin{array}{r}0.651 \\
.657 \\
.652 \\
.649 \\
.645 \\
.655\end{array}$ & $\begin{array}{l}0 \\
0 \\
0 \\
0 \\
0 \\
0\end{array}$ & $\begin{array}{l}0 \\
0 \\
7 \\
3 \\
3 \\
3\end{array}$ & $\begin{array}{l}0.0064 \\
.0017 \\
.0053 \\
.0020 \\
.0046 \\
.0054\end{array}$ & $\begin{array}{r}0.11 \\
.13 \\
.13 \\
.14\end{array}$ & $\begin{array}{l}209 \\
196 \\
229 \\
216\end{array}$ & 212 & $\begin{array}{r}0.03 \\
.03\end{array}$ & $\begin{array}{l}18,720 \\
18,900\end{array}$ & $\begin{array}{l}53.8 \\
66.4\end{array}$ & 60.1 & 3.53 \\
\hline \multicolumn{18}{|c|}{ 3/4 Inch American National Fine } \\
\hline $\begin{array}{l}1 \\
2 \\
2 \\
3 \\
4 \\
5 \\
6\end{array}$ & $\begin{array}{l}5 \\
5 \\
5 \\
5 \\
5 \\
5\end{array}$ & $\begin{array}{l}0.6571 \\
.6602 \\
.6590 \\
.6622 \\
.6610 \\
.6606\end{array}$ & $\begin{array}{r}-0.0054 \\
-.0066 \\
-.0034 \\
-.0046 \\
-.0050\end{array}$ & $\begin{array}{r}10.703 \\
.707 \\
.707 \\
.707 \\
.707 \\
.708\end{array}$ & $\begin{array}{r}0.073 \\
.028 \\
.017 \\
.073 \\
.000 \\
.000\end{array}$ & $\begin{array}{r}0.657 \\
.651 \\
.655 \\
.653 \\
.655 \\
.651\end{array}$ & $\begin{array}{l}0 \\
0 \\
0 \\
0 \\
0 \\
0\end{array}$ & $\begin{array}{l}3 \\
0 \\
0 \\
0 \\
7 \\
3\end{array}$ & $\begin{array}{l}0.0025 \\
.0052 \\
.0059 \\
.0055 \\
.0061 \\
.0047\end{array}$ & $\begin{array}{r}0.17 \\
.15 \\
.15 \\
.17\end{array}$ & $\begin{array}{l}257 \\
264 \\
286 \\
293\end{array}$ & 275 & $\begin{array}{l}0.06 \\
.05\end{array}$ & $\begin{array}{r}21,650 \\
21,540\end{array}$ & $\begin{array}{l}118 \\
101\end{array}$ & 110 & 2.50 \\
\hline
\end{tabular}

3/4 Inch Dardelet

\begin{tabular}{|c|c|c|c|c|c|c|c|c|c|c|c|c|c|c|c|c|c|}
\hline $\begin{array}{l}1 \\
12 \\
3 \\
4 \\
4 \\
5 \\
6\end{array}$ & $\begin{array}{l}5 \\
5 \\
2 \\
5 \\
5 \\
5\end{array}$ & $\begin{array}{r}0.6728 \\
.6724 \\
.6740 \\
.6728 \\
.6724 \\
.6740\end{array}$ & $\begin{array}{r}-0.0022 \\
-.0026 \\
-.0010 \\
-.0022 \\
-.0026 \\
-.0010\end{array}$ & 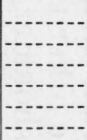 & $\begin{array}{r}0.140 \\
.151 \\
.140 \\
.179 \\
.157 \\
.112\end{array}$ & $\begin{array}{l}0.875 \\
.873 \\
.875 \\
.878 \\
.874 \\
.876\end{array}$ & $\begin{array}{l}0 \\
0 \\
0 \\
0 \\
0 \\
0\end{array}$ & $\begin{array}{l}13 \\
13 \\
20 \\
20 \\
27 \\
37\end{array}$ & $\begin{array}{l}0.0032 \\
.0023 \\
.0013 \\
.0027 \\
.0054 \\
.0032\end{array}$ & \begin{tabular}{r}
0.17 \\
.25 \\
.22 \\
.17 \\
\hdashline \\
\end{tabular} & $\begin{array}{l}286 \\
354 \\
370 \\
293\end{array}$ & 326 & $\begin{array}{r}0.06 \\
.06\end{array}$ & $\begin{array}{l}21,770 \\
22,130\end{array}$ & $\begin{array}{l}118 \\
134\end{array}$ & 126 & 2. 59 \\
\hline
\end{tabular}

${ }_{1}$ Did not comply with requirements. 
The thickness of each nut was measured using a micrometer caliper. All of the American National nuts, both coarse and fine, complied with the requirements as to thickness for semifinished regular nuts. No tolerances were given for the Dardelet nuts.

The obliquity of the bearing face of each nut was measured, using the fixture shown in figure 10 . The nuts were screwed on a slightly tapered mandrel, a, having a corresponding thread. The mandrel was placed on the centers, $b-b$, and the dial micrometer, $c$, was clamped so that the micrometer contact rested on the bearing face of the nut. The mandrel was then rotated by hand. The obliquity in degrees was computed from the difference between the maximum and minimum micrometer readings. If the difference was zero, the bearing face was perpendicular to the axis of the threads. All the nuts complied with the requirements for both semifinished and finished regular nuts.

The diametrical clearance between the bolt and nut was measured using the fixture shown in figure 11. The bolt was held firmly in the fixture by the clamping plate, a, and thumbscrew, b. The spindle of the dial micrometer rested on the nut directly over the axis of the bolt. A small upward force was applied to the nut by the fingers, while the nut was rotated back and forth through a small angle, the minimum reading being recorded. This operation was repeated with a small downward force applied to the nut. The difference between the two readings was taken as the diametrical clearance between the bolt and nut. In measuring the clearance between a bolt and nut having Dardelet threads the same procedure was followed except that a small axial force, toward the head of the bolt, was applied to the nut in addition to the upward and downward forces. This axial force kept the nut in the position of maximum clearance.

Insofar as could be determined from these measurements of clearance all of the American National specimens, both coarse and fine, complied with the requirements for pitch-diameter tolerance for class 3 , medium fit. No tolerances were given for the Dardelet specimens.

\section{METHOD OF TESTING}

\section{GENERAL}

As the results of impact tests nearly always show greater variations than the results of static tests, four of the six similar specimens of each material, thread, and size were tested under impact tensile loading and the other two under static tensile loading. The distance between the shoulder on the bolt and the bearing face of the nut was five times the nominal diameter of the bolt. In this distance, the thread was exposed one diameter from the face of the nut, as shown in figure 9.

\section{IMPACT TESTS}

The impact tests were made in the Charpy machine shown in figures 12, 13, and 14, located at Watertown Arsenal, Mass. The capacity was $2,170 \mathrm{ft}-\mathrm{lb}(300 \mathrm{~kg}-\mathrm{m})$.

As shown in figure 14 , the bolt, a, was screwed into the pendulum, $\mathrm{b}$, the spacer, c, and the collar, $\mathrm{d}$, were placed on the bolt, then the nut, e, was screwed on hand-tight. If the nuts on the Dardelet 


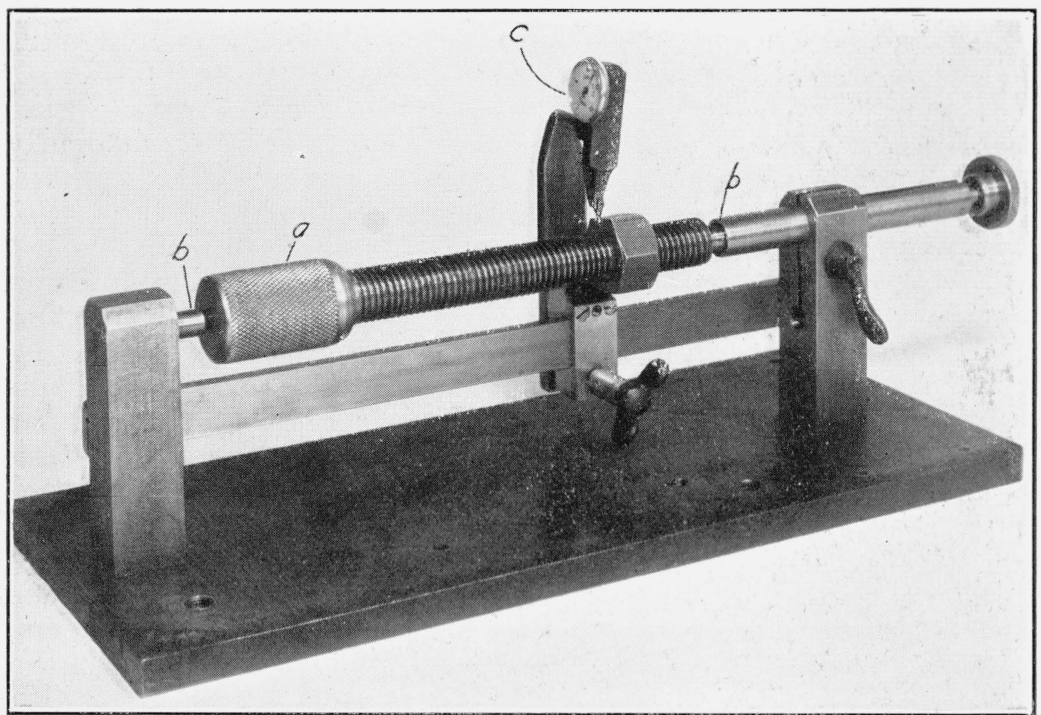

FiguRE 10.-Fixture used for measuring the obliquity of the face of the nut.

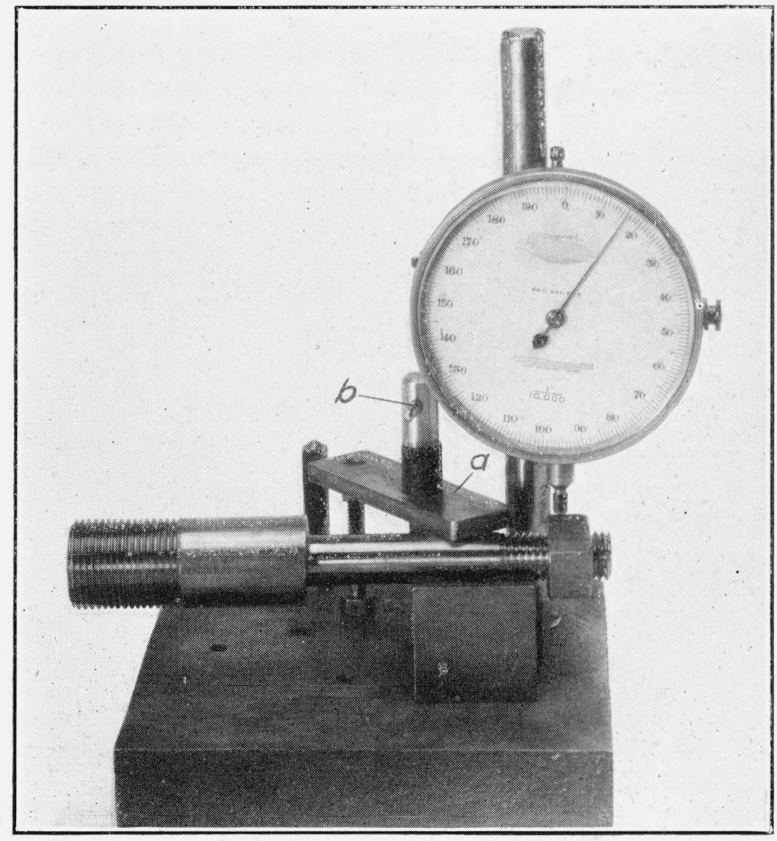

FIGURE 11.-Fixture used for measuring the diametrical clearance between the threads of the bolt and the threads of the nut. 


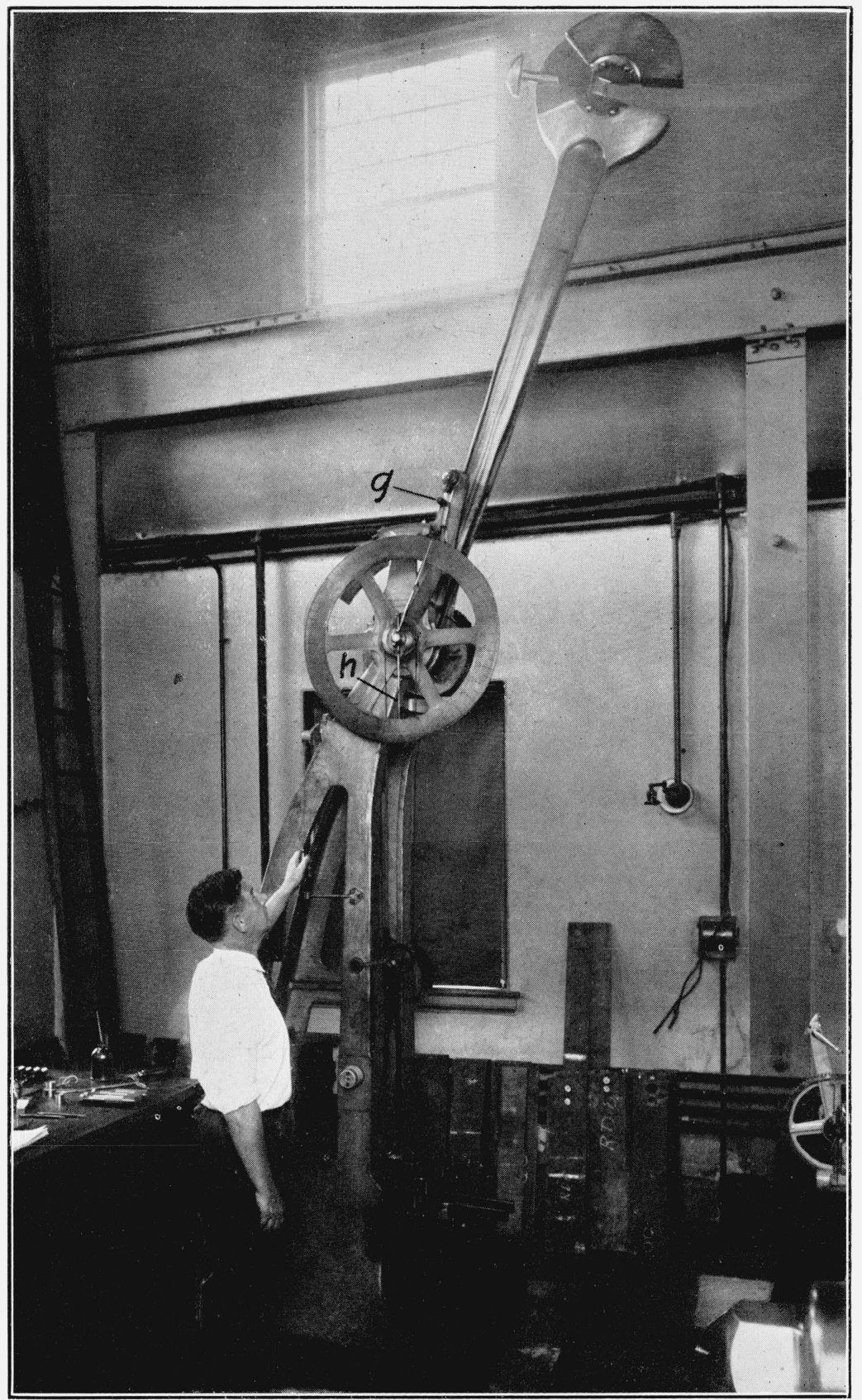

FIGURE 12.-Charpy impact machine, capacity 2,170 ft-lb (300 kg-m), pendulum raised. 


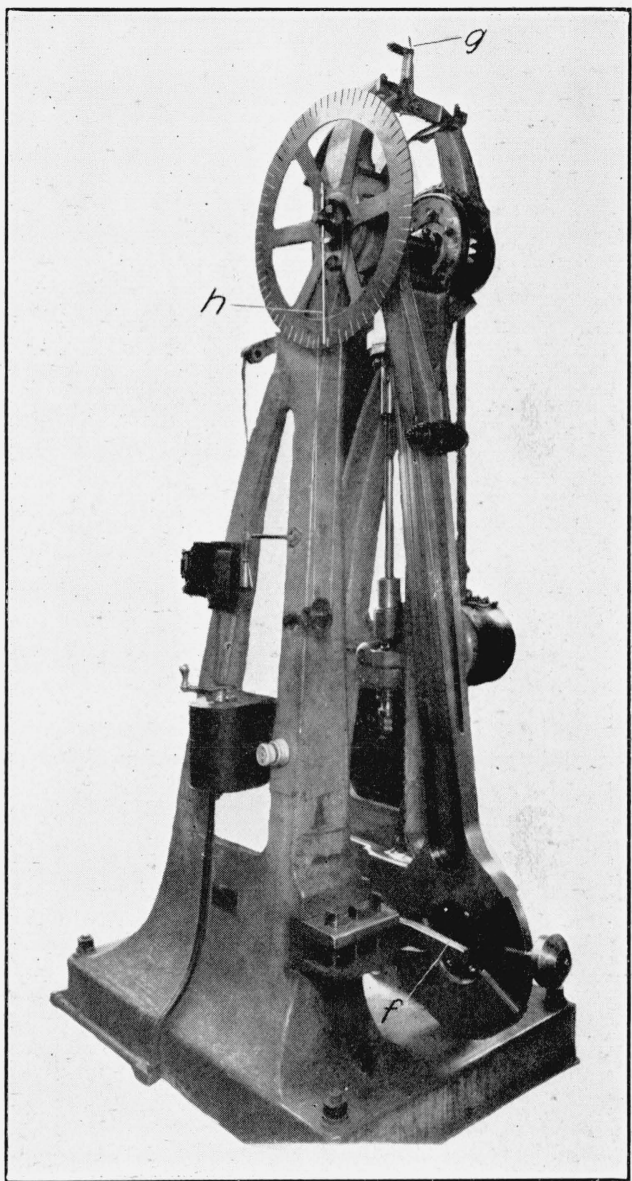

FIGURE 13.-Charpy impact machine, collar near the stops. 


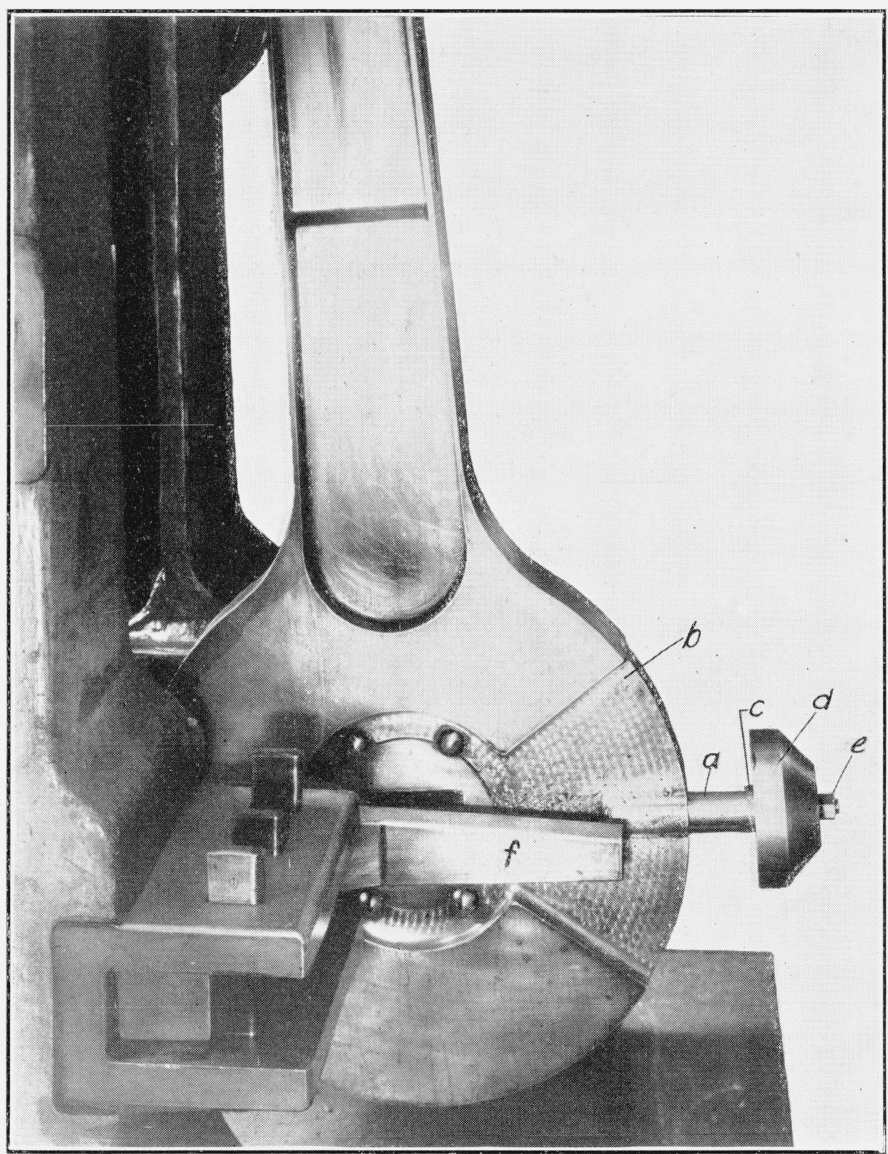

FIgURE 14.-Charpy impact machine, lower portion of pendulum with specimen in position. 


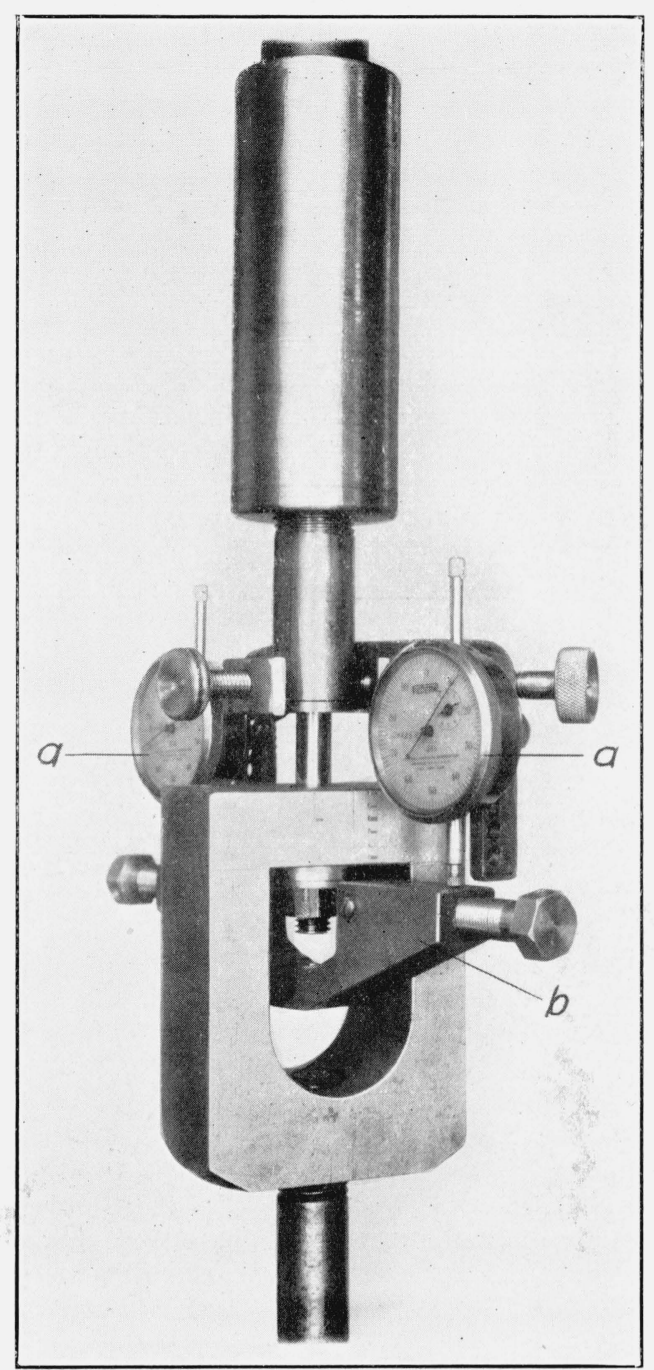

FiguRE 15.-Extensometer used for measuring elastic stretch in static tensile specimens. 


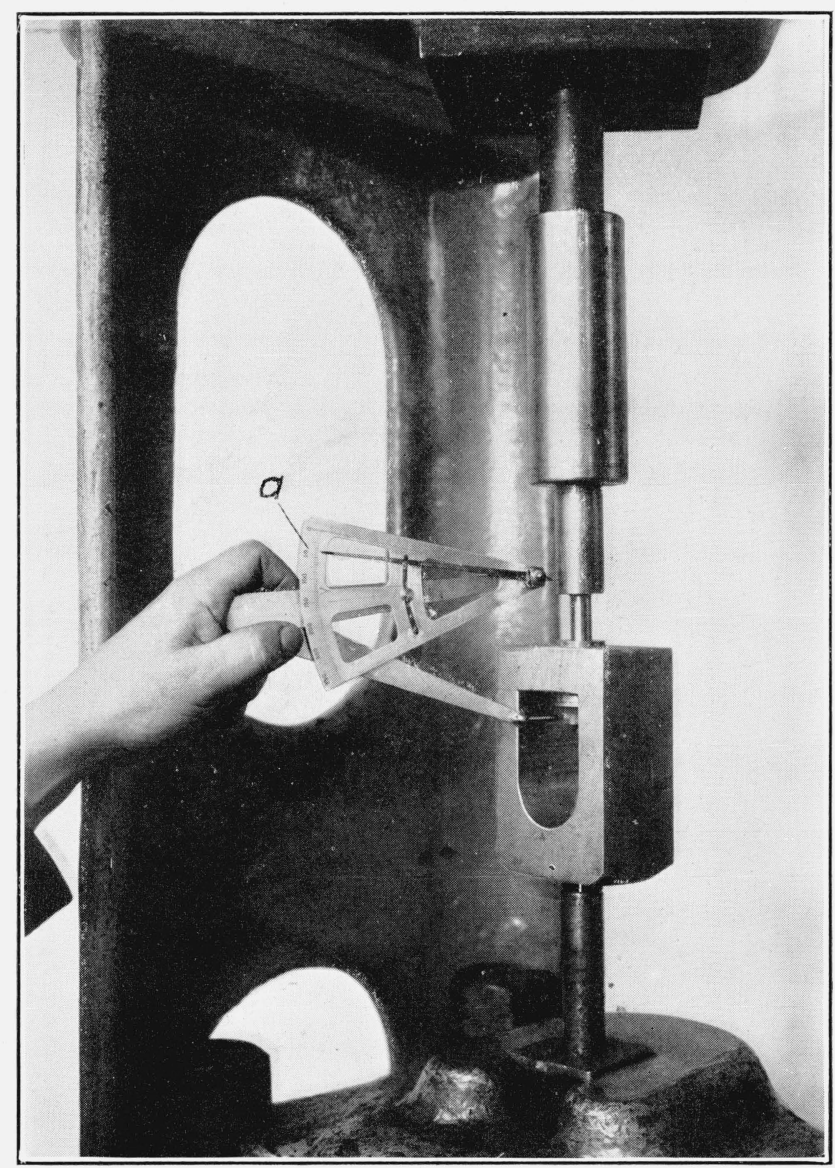

FIGURE 16.-Dividers used for measuring the stretch to rupture in static tensile specimens. 
specimens had been tightened as in actual use, the thrust faces of the threads would have been in contact. The work required to bring the thrust faces into contact was estimated from the load-stretch diagrams for the $1 / 2$-in. bolts. For the chromium-nickel steel and the Monel-metal specimens this work was less than 1 percent of the area of the diagram and, therefore, of the static work. For the bronze and the brass specimens, the work was less than $1 \frac{1}{2}$ percent and for the cold-rolled steel specimens less than $2 \frac{3}{4}$ percent. The values of the impact work should be decreased by about these percentages when estimating the impact work of Dardelet bolts which are under service tensile stress. The work required for American National bolts, both coarse and fine, to subject them to service tensile stress is much less than the work required for Dardelet bolts. Approximate computations based on the results of the static tests on $1 / 2$ in. bolts showed that this work was less than one-half of 1 percent of the static work. For some of the steel specimens it was less than 1 percent of the static work.

A different spacer, c, was used for the specimens of each nominal diameter. The spacer was machined so that the sum of its thickness and the thickness of the collar was equal within a tolerance of 0.02 in. to five times the nominal diameter of the bolt.

If there was a measurable angle between the axis of the large thread and the axis of the bolt, the bolt was rotated in the pendulum until the axis lay in a vertical plane. The collar, therefore, struck both stops, f, simultaneously. A prick punch was used to mark the position of the nut on the bolt.

The pendulum was raised until it engaged the latch release, g, figures 12 and 13, where it was held at an angle of $70^{\circ}$ above the horizontal. The pointer, h, was set at zero and the latch tripped, releasing the pendulum and allowing it to swing downward. The collar struck the stops and the specimen ruptured. Due to its residual energy, the pendulum continued past the vertical position. The pointer registered the maximum angle of swing after rupturing the bolt. This angle was read to the nearest $1 / 2{ }^{\circ}$ and the residual energy computed. The impact work required to rupture the specimen was obtained by subtracting the residual energy of the pendulum from its initial energy.

Some of the energy was expended in stretching the portion of the bolt which had not been turned down. The length of this portion differed for each size of specimen because the overall lengths of the bolts were the same and the length of the reduced portion was five times the nominal diameter or size. Approximate calculations based upon the materials having the highest ratio of ultimate strength to Young's modulus of the material and the greatest static load sustained by any specimen showed that this work could in no case exceed 2 percent of the total impact work for any of the $3 / 4 \mathrm{in}$. specimens. This percentage would decrease as the nominal diameter of the specimen decreased.

With the nut in the position indicated by the punch mark, the length of the ruptured specimens from the shoulder on the bolt to the bearing face of the nut was measured. The stretch was obtained by subtracting the initial distance (five times the nominal diameter of the bolt) from this distance. 


\section{STATIC TESTS}

The static specimens were tested in a screw-power, beam and poise testing machine having a capacity of $50,000 \mathrm{lb}$. The extensometer shown in figure 15 was attached to the specimen and simultaneous readings of the load and dials taken until the stretch increased much more rapidly than the load. The extensometer was then removed to prevent injuring the dial micrometers. Two micrometers, a-a, mounted on a collar were attached to the bolt by three screws, one-half of the diameter of the bolt above the shoulder. The clamp, b, was fastened by horizontal knife edges to the nut, one-half of the nominal diameter of the bolt below the bearing face of the nut. The gage length was therefore six times the nominal diameter of the bolt. The spindles of the micrometers rested on the horizontal upper surface of the clamp. The dial micrometers were calibrated by using a bench screw micrometer. It is believed that the error of the extensometer did not exceed 0.001 in.

The stretch obtained from the extensometer readings was taken as the stretch between the shoulder on the bolt and the face of the nut because the deformations at the shoulder of the bolt and of the nut were so small as to be negligible.

Before the extensometer was removed, the points of a modified divider shown in figure 16 were applied to the specimen and the stretch for several loads was read simultaneously on both extensometer and divider. The points of the divider were placed in prick punch marks on the specimen. These marks were in the horizontal planes at which the extensometer was attached. The stretch of the specimen was indicated on the scale, a, by the lever, which had a ratio of 10:1. This divider was calibrated using a bench screw micrometer. It is believed that the error of the readings of the divider did not exceed 0.005 in. Readings of the divider and corresponding loads were taken as the stretch increased until the specimen ruptured.

\section{RESULTS}

The results of the measurements and tests of the specimens are given in tables 5 to 9 , inclusive.

From the load-stretch readings, diagrams were plotted for each static specimen. Diagrams for one of the two duplicate specimens of all materials having nominal diameters of $1 / 2$ in. are shown in figure 17. These are typical of those for the other specimens. For all the specimens having American National fine and Dardelet threads, the stretch was greater than for those having American National coarse threads. For low loads the curves of the American National threads, coarse and fine, show that the stretch is approximately proportional to the load. For loads up to a few thousand pounds the stretch of the Dardelet specimens was much greater than that for the specimens having American National threads, due to axial movement of the nut on the bolt until the thrust faces came into contact. For higher loads, after the thrust faces came into contact, the stretch increased at about the same rate as for the specimens having American National threads. The static work was computed from the area under the load-stretch curve. 


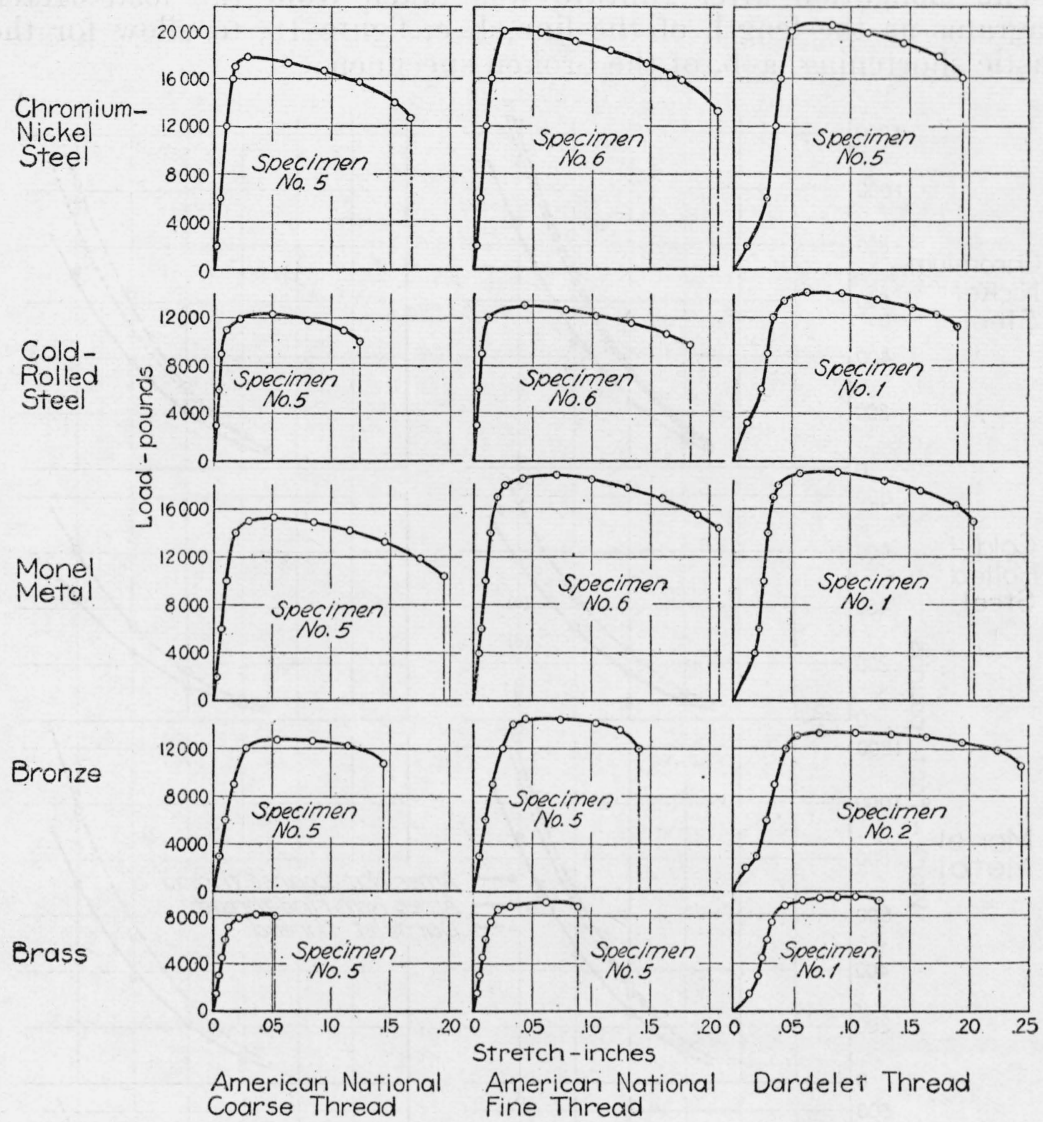

FIGURE 17. - Static load-stretch diagrams for $1 / 2-$ inch bolts.

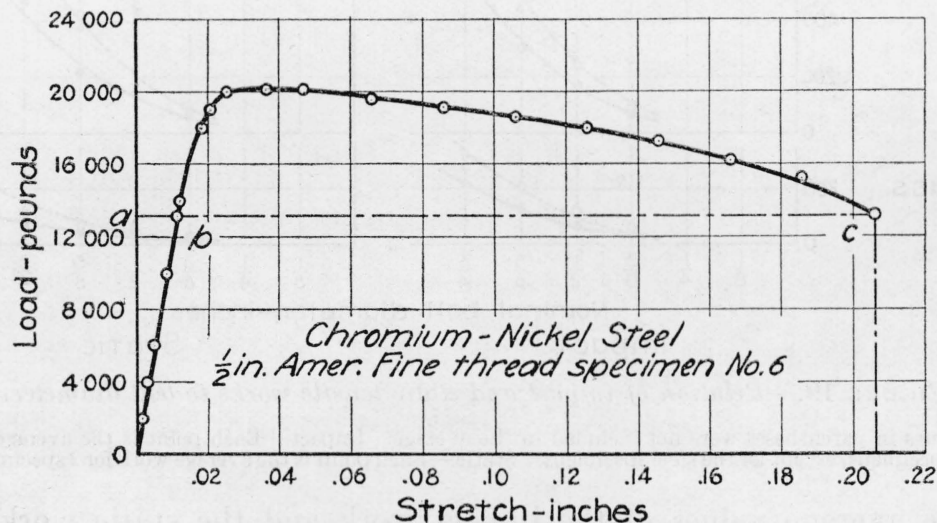

Figure 18.-Typical static load-stretch diagram.

The elongation after rupture was taken as the length of the line, b-c, to allow for the elastic shortening $\mathrm{a}-\mathrm{b}$, of the broken specimen. 
The elongation after rupture was taken from the load-stretch diagrams as the length of the line, b-c, figure 18, to allow for the elastic shortening, $\mathrm{a}-\mathrm{b}$, of the broken specimen.

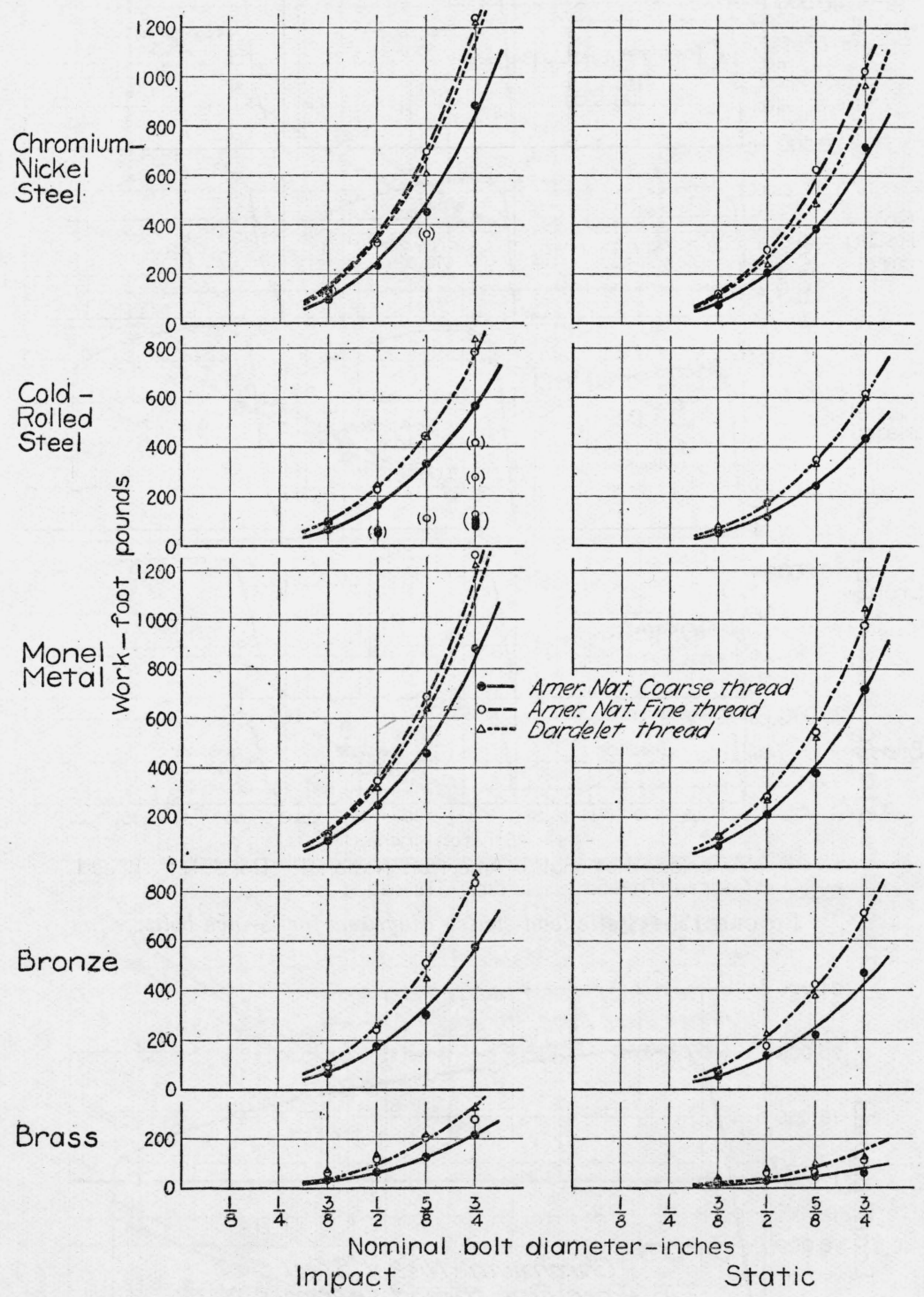

FIGURE 19.-Relation of impact and static tensile works to bolt diameter.

The values in parentheses were not included in the average. Impact.-Each point is the average work for 4 specimens, except for the steel specimens. Static.-Each point is the average work for 2 specimens.

The average values of the impact work and the static work are shown graphically in figure 19 , and the average values of the maximum static loads in figure 20 . 


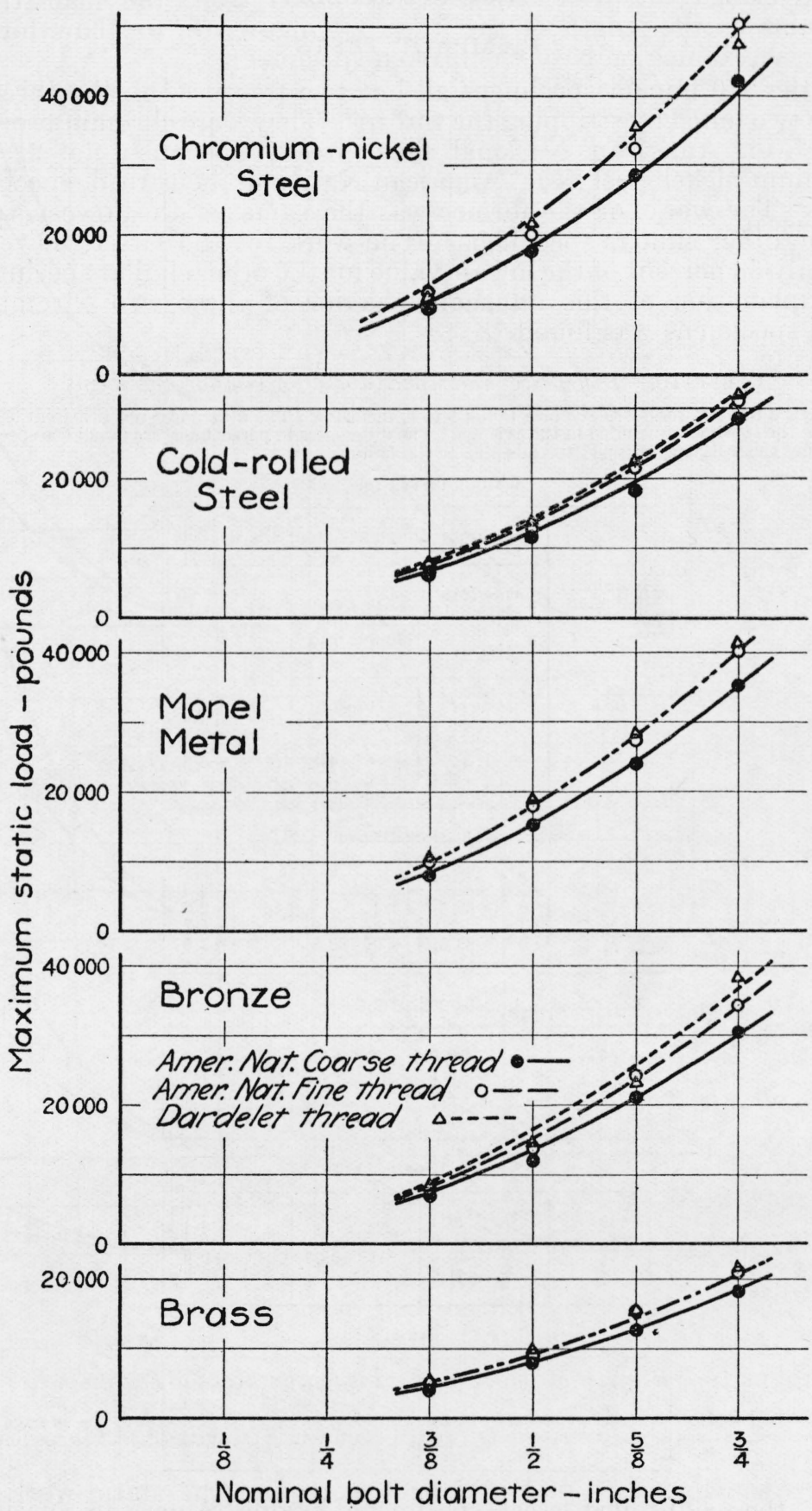

Figure 20.--Relation of maximum static loads to bolt diameters. Each point is the average maximum load for 2 specimens. 


\section{DISCUSSION}

\section{IMPACT TESTS}

Of the 240 impact specimens all but two fractured in the threads. These two failed by stripping the threads. They were chromium-nickel steel, $3 / 8$-in. American National fine thread, specimen no. 2, and chromium-nickel steel, 58 -in. American National fine thread, specimen no. 1. The work for the former was the same as the lowest value for the other similar specimens. The work for the latter, however, was only 53 percent of the lowest value for the other similar specimens. No explanation of this unusual behavior of these two chromiumnickel specimens was found.

TABLE 10.-Differences in impact works for similar specimens

[The average impact works are given in tables 5 to 9 , inclusive. The differences not in parentheses are only for the specimens included in the average. The differences in parentheses are for all the specimens including those having stripped threads and brittle failures.]

Chromium-Nickel Steel

\begin{tabular}{|c|c|c|c|}
\hline \multirow[b]{2}{*}{$\begin{array}{l}\text { Nominal } \\
\text { diameter }\end{array}$} & \multicolumn{3}{|c|}{ Maximum differences from the average } \\
\hline & $\begin{array}{c}\text { American } \\
\text { National } \\
\text { coarse } \\
\text { thread }\end{array}$ & $\begin{array}{c}\text { American } \\
\text { National } \\
\text { fine thread }\end{array}$ & $\begin{array}{c}\text { Dardelet } \\
\text { thread }\end{array}$ \\
\hline $\begin{array}{l}\text { Inch } \\
3 / 8 \\
1 / 2 \\
5 / 8 \\
3 / 4\end{array}$ & $\begin{array}{r}\text { Percent } \\
+6.4 \\
+2.2 \\
+7.5 \\
-3.1\end{array}$ & $\begin{aligned} & \text { Percent } \\
- & 3.0(-3.0) \\
- & 2.8 \\
+ & 3.6(-47.9) \\
+ & 4.6\end{aligned}$ & $\begin{array}{l}\text { Percent } \\
+5.3 \\
\pm 2.4 \\
-8.3 \\
-6.9\end{array}$ \\
\hline
\end{tabular}

Cold-Rolled Steel

\begin{tabular}{|l|l|l|l|}
\hline $3 / 5$ & -5.9 & +3.3 & \pm 5.3 \\
$1 / 2$ & $0.0(-f .9 .5)$ & \pm 4.4 & \pm 2.9 \\
$5 / 8$ & \pm 1.5 & $\pm 2.5(-75.0)$ & \pm 5.7 \\
$3 / 4$ & $0.0(-86.0)$ & $0.0(-83.3)$ & +2.6 \\
\hline
\end{tabular}

Monel Metal

\begin{tabular}{|l|r|r|r|}
\hline $3 / 8$ & 0.0 & \pm 2.3 & \pm 2.3 \\
$1 / 2$ & -3.7 & \pm 2.9 & \pm .6 \\
$5 / 8$ & +2.9 & -8.2 & \pm 1.4 \\
$3 / 4$ & -2.7 & -2.5 & +1.6 \\
\hline
\end{tabular}

\begin{tabular}{|c|c|c|c|}
\hline $\begin{array}{l}3 / 8 \\
1 / 2 \\
5 / 8 \\
3 / 4\end{array}$ & $\begin{array}{r}-19.4 \\
-4.0 \\
+19.9 \\
+21.6\end{array}$ & $\begin{array}{r}+8.7 \\
-5.0 \\
-13.1 \\
-5.5\end{array}$ & $\begin{array}{r}-3.1 \\
-5.4 \\
+9.0 \\
-18.6\end{array}$ \\
\hline \multicolumn{4}{|c|}{ Brass } \\
\hline $\begin{array}{l}3 / 8 \\
1 / 2 \\
5 / 8 \\
3 / 4\end{array}$ & $\begin{array}{r}+12.1 \\
-9.1 \\
+8.1 \\
+8.0\end{array}$ & $\begin{array}{r}0.0 \\
-18.8 \\
+3.5 \\
\pm 6.5\end{array}$ & $\begin{array}{r}+6.1 \\
-5.9 \\
-18.0 \\
+13.5\end{array}$ \\
\hline
\end{tabular}

For the cold-rolled steel specimens having American National threads, coarse and fine, the impact works were very much more erratic than those for the specimens of other materials. For ten of the cold-rolled specimens the fracture was like that of a brittle 
material and the impact work was much lower than that of the similar specimens. Typical specimens are shown in figure 21. For each pair the one on the left had a brittle failure and the one on the right a ductile failure. It is evident that the reduction of area was much greater for the specimens on the right. Metallurgical examination of these cold-rolled steel specimens gave no conclusive reason for the differences in behavior. A careful study of the threads on representative specimens, particularly of the minor diameter and the profile and surface of the thread at the root, suggested no explanation. However, the fact that none of the Dardelet specimens showed brittle failures suggests that there might have been a notch effect under impact loading for the specimens having American National threads.

The maximum percentage differences from the average impact works for each group of similar specimens are given in table 10 . As noted above, very great differences were found in the brittle failures of the cold-rolled steel, and in one of the stripped-thread failures of the chromium-nickel steel.

Excluding these failures, all of which occurred in specimens having American National threads, the remaining differences were about the same for all three types of thread.

The bronze and brass specimens were the least uniform.

The ratios of the average impact works are given in table 11 . For all of the materials, the impact work for bolts having American National coarse threads was less than the impact work for bolts of the same size and material having American National fine threads. The ratios for all of the bolts except the brass bolts were about the same. The ratios for the brass bolts were somewhat lower than for bolts of the other materials.

TABLE 11.-Ratios of impact works

\begin{tabular}{|c|c|c|c|c|}
\hline \multicolumn{2}{|c|}{ Specimen } & \multicolumn{3}{|c|}{ Ratios of average impact work } \\
\hline Nominal diameter & Material & $\begin{array}{l}\text { American } \\
\text { National } \\
\text { coarse: } \\
\text { American } \\
\text { National fine }\end{array}$ & $\begin{array}{l}\text { American } \\
\text { National } \\
\text { fine: Dar- } \\
\text { delet }\end{array}$ & $\begin{array}{l}\text { Dardelet: } \\
\text { American } \\
\text { National } \\
\text { coarse }\end{array}$ \\
\hline Inch & 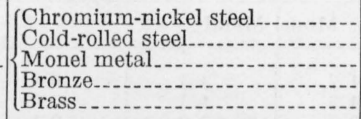 & $\begin{array}{r}10.71 \\
.74 \\
.76 \\
.67 \\
.55\end{array}$ & $\begin{array}{r}10.99 \\
.97 \\
1.00 \\
.94 \\
.91\end{array}$ & $\begin{array}{l}1.42 \\
1.40 \\
1.31 \\
1.58 \\
1.97\end{array}$ \\
\hline $1 / 2$ & $\left\{\begin{array}{l}\text { Chromium-nickel steel } \\
\text { Cold-rolled steel__. } \\
\text { Monel metal_. } \\
\text { Bronze._. } \\
\text { Brass }\end{array}\right.$ & $\begin{array}{r}.71 \\
.73 \\
.71 \\
.73 \\
.56\end{array}$ & $\begin{array}{r}.96 \\
.93 \\
1.09 \\
.94 \\
.86\end{array}$ & $\begin{array}{r}1.46 \\
21.48 \\
1.29 \\
1.45 \\
2.06\end{array}$ \\
\hline $58 .-$ & $\left\{\begin{array}{l}\text { Chromium-nickel steel } \\
\text { Cold-rolled steel } \\
\text { Monel metal } \\
\text { Bronze } \\
\text { Brass }\end{array}\right.$ & $\begin{array}{r}1.65 \\
.74 \\
.66 \\
.59 \\
.61\end{array}$ & $\begin{array}{r}11.15 \\
21.02 \\
1.07 \\
1.15 \\
.93\end{array}$ & $\begin{array}{l}1.34 \\
1.31 \\
1.41 \\
1.47 \\
1.74\end{array}$ \\
\hline & $\left\{\begin{array}{l}\text { Chromium-nickel steel } \\
\text { Cold-rolled steel_- } \\
\text { Monel metal_- } \\
\text { Bronze } \\
\text { Brass }\end{array}\right.$ & $\begin{array}{r}.71 \\
2.73 \\
.70 \\
.69 \\
.77\end{array}$ & $\begin{array}{r}1.02 \\
2.92 \\
1.03 \\
.95 \\
.84\end{array}$ & $\begin{array}{r}1.38 \\
21.48 \\
1.39 \\
1.53 \\
1.54\end{array}$ \\
\hline A verage for all diameters... & 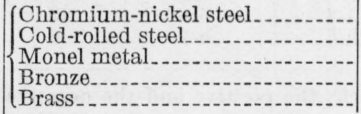 & $\begin{array}{r}1.70 \\
.74 \\
.71 \\
.67 \\
.62\end{array}$ & $\begin{array}{r}11.03 \\
2.96 \\
1.05 \\
1.00 \\
.88\end{array}$ & $\begin{array}{rl} & 1.40 \\
2 & 1.42 \\
1.35 \\
1.51 \\
1.83\end{array}$ \\
\hline
\end{tabular}

1 Stripped threads not included in the average (see table 5).

2 Brittle failures not included in average_(see table 6). 
The impact work for bolts having American National fine threads was sometimes greater and sometimes less than for bolts having Dardelet threads.

For all of the materials the impact work for bolts having Dardelet threads was greater than the impact work for bolts of the same size and material having American National coarse threads. The ratios for the brass bolts were considerably greater than the ratios for the bolts of the other materials.

\section{STATIC TESTS}

Of the 120 static specimens, all fractured in the bolt threads. There were no brittle failures and in no case did the threads strip.

The maximum percentage differences from the average static works for similar specimens are given in table 12. For each of the threads these differences were small for the chromium-nickel steel, cold-rolled steel, and monel metal specimens. The differences for the bronze and the brass bolts were considerably greater.

TABLE 12.-Differences in static works for similar specimens

\begin{tabular}{|c|c|c|c|}
\hline \multicolumn{4}{|c|}{ Chromium-Nickel Steel } \\
\hline \multirow{3}{*}{$\begin{array}{l}\text { Nominal } \\
\text { diameter }\end{array}$} & \multicolumn{3}{|c|}{ Differences from the average 1} \\
\hline & \multicolumn{2}{|c|}{ American National } & \multirow{2}{*}{$\begin{array}{c}\text { Dardelet } \\
\text { thread }\end{array}$} \\
\hline & Coarse thread & Fine thread & \\
\hline $\begin{array}{l}\text { Inch } \\
3 / 8 \\
1 / 2 \\
5 / 8 \\
3 / 4\end{array}$ & $\begin{array}{c}\text { Percent } \\
1.3 \\
5.4 \\
.3 \\
1.3\end{array}$ & $\begin{array}{c}\text { Percent } \\
1.7 \\
1.7 \\
.2 \\
10.1\end{array}$ & $\begin{array}{c}\text { Perceut } \\
0.9 \\
9.1 \\
2.9 \\
3.7\end{array}$ \\
\hline \multicolumn{4}{|c|}{ Cold-Rolled Steel } \\
\hline $\begin{array}{l}3 / 8 \\
1 / 2 \\
5 / 8 \\
3 / 4\end{array}$ & $\begin{array}{l}0.2 \\
2.5 \\
5.3 \\
1.4\end{array}$ & $\begin{array}{r}0.3 \\
1.1 \\
2.0 \\
.8\end{array}$ & $\begin{array}{l}\text { 1. } 2 \\
\text { 3. } 3 \\
3.9 \\
3.2\end{array}$ \\
\hline \multicolumn{4}{|c|}{ Monel Metal } \\
\hline $\begin{array}{l}3 / 8 \\
1 / 2 \\
5 / 8 \\
3 / 4\end{array}$ & $\begin{array}{r}1.2 \\
4.3 \\
2.7 \\
.3\end{array}$ & $\begin{array}{r}3.3 \\
4.3 \\
3.0 \\
.1\end{array}$ & $\begin{array}{l}0.8 \\
2.6 \\
2.1 \\
1.4\end{array}$ \\
\hline \multicolumn{4}{|c|}{ Bronze } \\
\hline $\begin{array}{l}3 / 8 \\
1 / 2 \\
5 / 8 \\
3 / 4\end{array}$ & $\begin{array}{r}15.7 \\
2.2 \\
19.4 \\
27.5\end{array}$ & $\begin{array}{r}0.5 \\
15.9 \\
21.6 \\
25.9\end{array}$ & $\begin{array}{r}5.1 \\
.9 \\
23.1 \\
25.6\end{array}$ \\
\hline \multicolumn{4}{|c|}{ Brass } \\
\hline $\begin{array}{l}3 / 8 \\
1 / 2 \\
5 / 8 \\
3 / 4\end{array}$ & $\begin{array}{r}22.1 \\
17.0 \\
3.2 \\
10.5\end{array}$ & $\begin{array}{r}32.1 \\
3.3 \\
6.8 \\
8.2\end{array}$ & $\begin{array}{r}6.4 \\
4.7 \\
12.9 \\
6.3\end{array}$ \\
\hline
\end{tabular}

${ }^{1}$ As there were two similar static specimens the positive and the negative differences were the same numerically. 


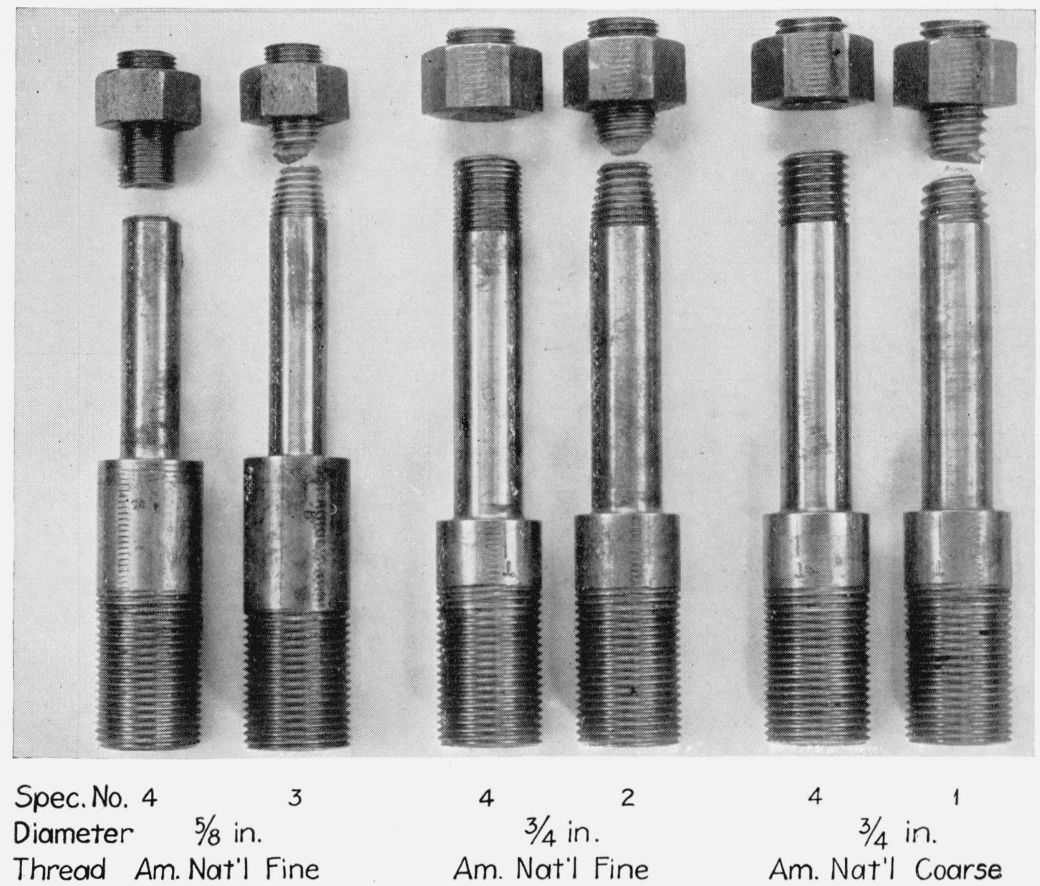

Figure 21.-Cold-rolled steel specimens showing, for each pair, typical brittle failures on the left and typical ductile failures on the right. 
The ratios of the average static works and of the average maximum static tensile loads are given in table 13 . For all of the materials the static work for bolts having American National coarse threads was less than the static work for bolts of the same size and material having American National fine threads. The ratios for all of the bolts except the brass bolts were about the same. The ratios for the brass bolts were somewhat lower than for bolts of the other materials. The static work for bolts having American National fine threads w as sometimes greater and sometimes less than for bolts having Dardelet threads. For all of the materials the static work for bolts having Dardelet threads was greater than the static work for bolts of the same size and material having American National coarse threads. The ratios for the brass bolts were much greater than the ratios for the bolts of the other materials.

TABLE 13.-Ratios of staitic works and maximum static loads

\begin{tabular}{|c|c|c|c|c|c|c|c|}
\hline \multicolumn{2}{|r|}{ Specimen } & \multicolumn{3}{|c|}{ Ratios of average static work } & \multicolumn{3}{|c|}{$\begin{array}{l}\text { Ratios of average maximum } \\
\text { static loads }\end{array}$} \\
\hline $\begin{array}{l}\text { Nominal } \\
\text { diameter }\end{array}$ & Material & $\begin{array}{l}\text { American } \\
\text { National } \\
\text { coarse: } \\
\text { American } \\
\text { National } \\
\text { fine }\end{array}$ & $\begin{array}{l}\text { American } \\
\text { National } \\
\text { fine: } \\
\text { Dardelet }\end{array}$ & $\begin{array}{l}\text { Dardelet: } \\
\text { American } \\
\text { National } \\
\text { coarse }\end{array}$ & $\begin{array}{l}\text { American } \\
\text { National } \\
\text { coarse: } \\
\text { American } \\
\text { National } \\
\text { fine }\end{array}$ & $\begin{array}{l}\text { American } \\
\text { National } \\
\text { fine: } \\
\text { Dardelet }\end{array}$ & $\begin{array}{l}\text { Darde- } \\
\text { let: } \\
\text { American } \\
\text { National } \\
\text { coarse }\end{array}$ \\
\hline Inch & $\left\{\begin{array}{l}\text { Chromium-nickel steel } \\
\text { Cold-rolled steel } \\
\text { Monel metal } \\
\text { Bronze.... } \\
\text { Brass_... }\end{array}\right.$ & $\begin{array}{l}0.65 \\
.67 \\
.68 \\
.69 \\
.55\end{array}$ & $\begin{array}{r}1.03 \\
.93 \\
.99 \\
.94 \\
.69\end{array}$ & $\begin{array}{l}\text { 1. } 50 \\
\text { 1. } 60 \\
\text { 1. } 48 \\
\text { 1. } 54 \\
\text { 2. } 63\end{array}$ & $\begin{array}{r}0.82 \\
.88 \\
.82 \\
.90 \\
.80\end{array}$ & $\begin{array}{r}1.04 \\
.98 \\
1.00 \\
.94 \\
1.02\end{array}$ & $\begin{array}{l}1.17 \\
1.16 \\
1.22 \\
1.18 \\
1.23\end{array}$ \\
\hline $1 / 2$ & $\left\{\begin{array}{l}\text { Chromium-nickel steel } \\
\text { Cold-rolled steel } \\
\text { Monel metal } \\
\text { Bronze... } \\
\text { Brass. }\end{array}\right.$ & $\begin{array}{l}.68 \\
.66 \\
.74 \\
.76 \\
.43\end{array}$ & $\begin{array}{r}1.25 \\
.99 \\
1.06 \\
.77 \\
.78\end{array}$ & $\begin{array}{l}\text { 1. } 18 \\
\text { 1. } 53 \\
\text { 1. } 27 \\
\text { 1. } 70 \\
\text { 2. } 94\end{array}$ & $\begin{array}{l}.88 \\
.91 \\
.85 \\
.86 \\
.89\end{array}$ & $\begin{array}{l}.94 \\
.97 \\
.99 \\
.57 \\
.93\end{array}$ & $\begin{array}{l}1.20 \\
1.13 \\
1.20 \\
1.19 \\
1.21\end{array}$ \\
\hline $5 / 8$ & $\left\{\begin{array}{l}\text { Chromium-nickel steel } \\
\text { Cold-rolled steel } \\
\text { Monel metal } \\
\text { Bronze. } \\
\text { Brass_. }\end{array}\right.$ & $\begin{array}{l}.61 \\
.70 \\
.69 \\
.52 \\
.67\end{array}$ & $\begin{array}{l}1.29 \\
1.05 \\
1.05 \\
1.12 \\
.66\end{array}$ & $\begin{array}{l}\text { 1. } 26 \\
\text { 1. } 35 \\
\text { 1. } 38 \\
\text { 1. } 70 \\
\text { 2. } 24\end{array}$ & $\begin{array}{l}.89 \\
.85 \\
.87 \\
.88 \\
.84\end{array}$ & $\begin{array}{r}.91 \\
.99 \\
.98 \\
1.02 \\
1.00\end{array}$ & $\begin{array}{l}1.24 \\
1.19 \\
1.16 \\
1.12 \\
1.19\end{array}$ \\
\hline $3 / 4$ & $\left\{\begin{array}{l}\text { Chromium-nickel steel } \\
\text { Cold-rolled steel } \\
\text { Monel metal } \\
\text { Bronze.... } \\
\text { Brass... }\end{array}\right.$ & $\begin{array}{l}.70 \\
.70 \\
.73 \\
.66 \\
.55\end{array}$ & $\begin{array}{r}1.07 \\
1.03 \\
.93 \\
1.03 \\
.87\end{array}$ & $\begin{array}{l}\text { 1. } 35 \\
\text { 1. } 39 \\
\text { 1. } 46 \\
\text { 1. } 46 \\
\text { 2. } 10\end{array}$ & $\begin{array}{l}.83 \\
.92 \\
.88 \\
.87 \\
.87\end{array}$ & $\begin{array}{r}1.06 \\
.99 \\
.98 \\
.90 \\
.98\end{array}$ & $\begin{array}{l}1.13 \\
1.10 \\
1.17 \\
1.28 \\
1.17\end{array}$ \\
\hline $\begin{array}{r}\text { Average } \\
\text { for all } \\
\text { diameters }\end{array}$ & $\left\{\begin{array}{l}\text { Chromium-nickel steel } \\
\text { Cold-rolled steel } \\
\text { Monel metal } \\
\text { Bronze.... } \\
\text { Brass. }\end{array}\right.$ & $\begin{array}{l}.66 \\
.68 \\
.71 \\
.66 \\
.55\end{array}$ & $\begin{array}{l}1.16 \\
1.00 \\
1.01 \\
.96 \\
.75\end{array}$ & $\begin{array}{l}\text { 1. } 32 \\
\text { 1. } 47 \\
\text { 1. } 40 \\
\text { 1. } 60 \\
\text { 2. } 48\end{array}$ & $\begin{array}{l}.86 \\
.89 \\
.86 \\
.88 \\
.85\end{array}$ & $\begin{array}{l}.98 \\
.98 \\
.99 \\
.96 \\
.98\end{array}$ & $\begin{array}{l}1.18 \\
1.14 \\
1.19 \\
1.19 \\
1.20\end{array}$ \\
\hline
\end{tabular}

For all of the materials, the maximum static load for bolts having American National coarse threads was less than the load for bolts of the same size and material having American National fine threads. The maximum static loads for bolts having American National fine threads were about the same as those for bolts having Dardelet threads. For all of the materials, the maximum static load for bolts having Dardelet threads was greater than the load for bolts of the same size and material having American National coarse threads. 
The ratios for the maximum static loads for all of the materials were about the same.

The static efficiency of a bolt may be defined as the ratio of the maximum tensile load of the bolt to the maximum tensile load of the shank of the bolt. The latter was computed by multiplying the ultimate strength of the material by the cross-sectional area of the shank. The average bolt efficiencies and the ratios of the area at the root of the thread to the area of the shank are given in table 14 . For all of the materials the bolt efficiency is greater than the ratios of the areas, showing that the tensile strength of the threaded portion of the bolts was greater than that of a cylindrical specimen having a diameter the same as the minor diameter of the bolt.

TABLE 14.-Static bolt efficiencies

\begin{tabular}{|c|c|c|c|c|c|c|c|}
\hline \multirow{3}{*}{$\begin{array}{l}\text { Nominal } \\
\text { diameter }\end{array}$} & \multirow{3}{*}{ Thread } & \multicolumn{5}{|c|}{ Material } & \multirow{3}{*}{$\frac{\text { Root area, }}{\text { shank area }}$} \\
\hline & & $\begin{array}{c}\text { Chrome- } \\
\text { nickel } \\
\text { steel }\end{array}$ & $\begin{array}{l}\text { Cold- } \\
\text { rolled } \\
\text { steel }\end{array}$ & $\begin{array}{l}\text { Monel } \\
\text { metal }\end{array}$ & Bronze & Brass & \\
\hline & & \multicolumn{5}{|c|}{ Bolt efficiency ! } & \\
\hline $\begin{array}{l}\text { Inch } \\
\quad 3 / 8\end{array}$ & $\left\{\begin{array}{l}\text { American National coarse } \\
\text { American National fine. } \\
\text { Dardelet.. }\end{array}\right.$ & $\begin{array}{r}\text { Percent } \\
65.3 \\
79.2 \\
76.4\end{array}$ & $\begin{array}{r}\text { Percent } \\
70.6 \\
80.2 \\
82.2\end{array}$ & $\begin{array}{r}\text { Percent } \\
70.3 \\
85.3 \\
85.7\end{array}$ & $\begin{array}{r}\text { Percent } \\
70.3 \\
78.0 \\
83.2\end{array}$ & $\begin{array}{r}\text { Percent } \\
65.2 \\
81.5 \\
80.2\end{array}$ & $\begin{array}{r}\text { Percent } \\
61.4 \\
73.2 \\
75.1\end{array}$ \\
\hline $1 / 2$ & $\left\{\begin{array}{l}\text { American National coarse } \\
\text { American National fine. } \\
\text { Dardelet. }\end{array}\right.$ & $\begin{array}{l}69.1 \\
78.1 \\
83.2\end{array}$ & $\begin{array}{l}73.5 \\
80.5 \\
83.1\end{array}$ & $\begin{array}{l}74.3 \\
87.9 \\
88.9\end{array}$ & $\begin{array}{l}67.3 \\
77.9 \\
79.9\end{array}$ & $\begin{array}{l}71.1 \\
79.7 \\
85.8\end{array}$ & $\begin{array}{l}64.0 \\
75.6 \\
77.4\end{array}$ \\
\hline $5 / 8$ & $\left\{\begin{array}{l}\text { American National coarse } \\
\text { American National fine } \\
\text { Dardelet. }\end{array}\right.$ & $\begin{array}{l}71.7 \\
80.9 \\
88.5\end{array}$ & $\begin{array}{l}72.3 \\
85.5 \\
86.1\end{array}$ & $\begin{array}{l}73.2 \\
83.8 \\
85.1\end{array}$ & $\begin{array}{l}75.8 \\
86.6 \\
84.6\end{array}$ & $\begin{array}{l}72.1 \\
85.8 \\
86.0\end{array}$ & $\begin{array}{l}65.8 \\
78.2 \\
77.4\end{array}$ \\
\hline $3 / 4$ & $\left\{\begin{array}{l}\text { American National coarse } \\
\text { American National fine. } \\
\text { Dardelet. }\end{array}\right.$ & $\begin{array}{l}73.0 \\
88.0 \\
82.8\end{array}$ & $\begin{array}{l}78.4 \\
85.2 \\
86.3\end{array}$ & $\begin{array}{l}74.4 \\
85.0 \\
87.0\end{array}$ & $\begin{array}{l}75.0 \\
86.0 \\
95.7\end{array}$ & $\begin{array}{l}72.7 \\
83.4 \\
84.8\end{array}$ & $\begin{array}{l}68.4 \\
79.5 \\
81.0\end{array}$ \\
\hline
\end{tabular}

1 The ratio of the maximum tensile load of the bolt to the computed maximum tensile load of the shank of the bolt.

\section{COMPARISON OF IMPACT AND STATIC PROPERTIES}

Inspection of the broken specimens showed that most of the stretch occurred in the threaded portion one diameter in length between the bearing face of the nut and the shoulder of the bolt. Consequently, most of the work was absorbed by this portion. The work absorbed would be expected to be proportional to the product of this "effective length" by some area approximating the area at the root of the thread. For comparison it was decided to compute the "specific work" for both impact and static specimens as the ratio of the average values of the work given in tables 5 to 9 by the "effective root volume" taken as the length, 1 nominal diameter, times the cross-sectional area at the root of the thread computed from the nominal minor diameter. The specific work for the impact and the static specimens was then obtained by dividing the average values of the work by the effective root volume. The "effective stretch" was obtained by dividing the average stretch for similar specimens by the nominal diameter. The values for specific work and effective stretch are shown in figure 22. 
The average values, for each material, of effective stretch and specific work for the bolts having American National fine threads are about the same as those for bolts having Dardelet threads. Except for the brass bolts the values for each material and type of thread

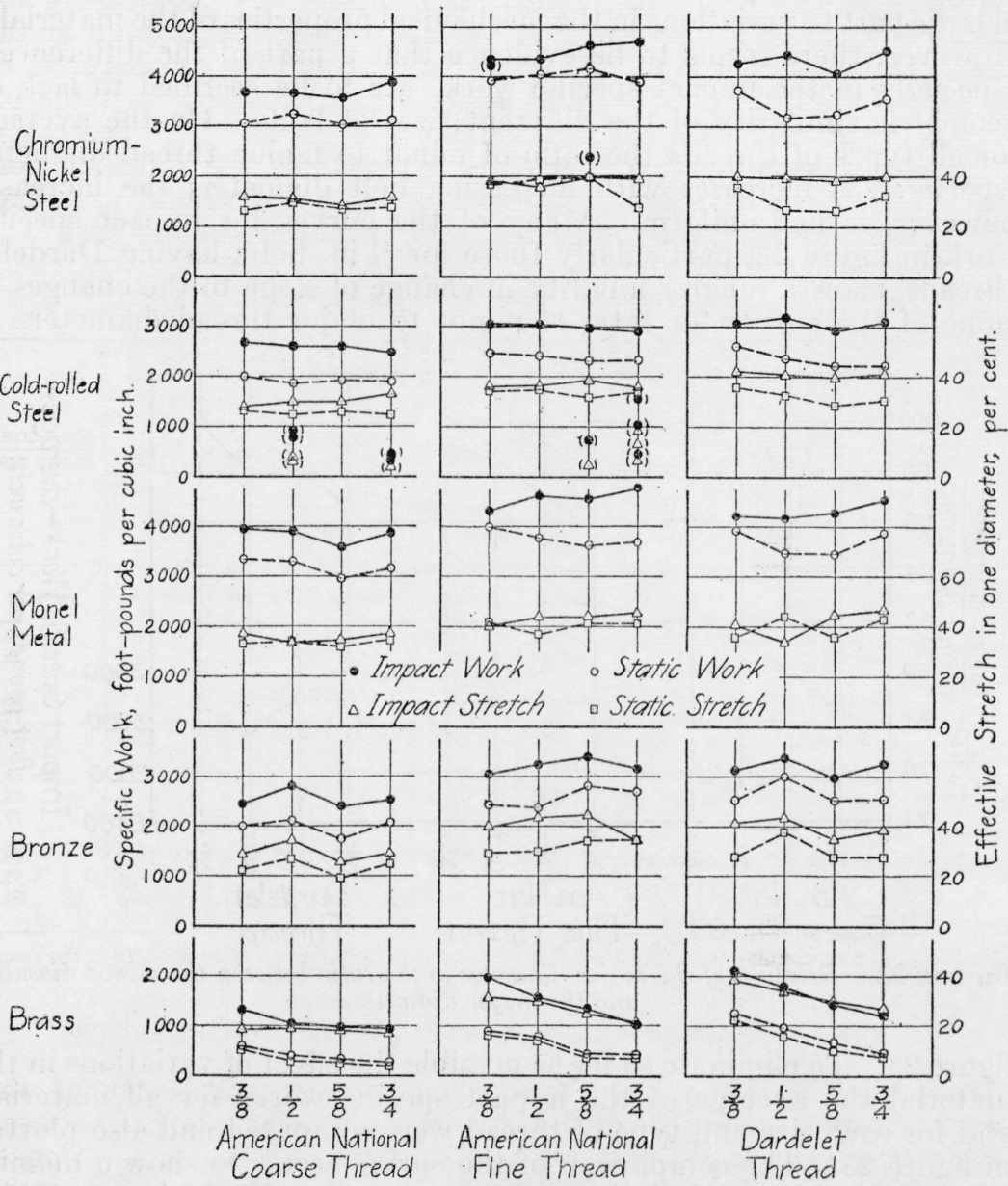

FIGURE 22.-Relation between the size of the bolt and the specific work and effective stretch.

The values in parentheses were not included in the average.

are about the same for the different bolt diameters. For the brass bolts the decrease in these values with an increase in the nominal diameter of the bolt probably is due to the fact that the hardness of the brass bars for the bolts decreased continuously toward the axis of the bar. Vickers numbers at points spaced $1 / 16$ in. along two mutually perpendicular diameters on cross sections of the brass bars showed that the center was much softer than the cylindrical surface. The amount of the harder material was greater for the bolts having the larger diameters than for those having the smaller diameters. Some of the other materials also were somewhat softer at the center but the 
differences were not nearly so great as for the brass. Six of the 8 bars of chromium-nickel steel were somewhat harder at the center, which may explain the small increase in impact specific work for these bolts with an increase in diameter.

The irregularities in the curves shown in figure 22 are probably due in large part to variations in the mechanical properties of the materials. However, there seems to be evidence that a part of the differences, especially in the impact specific work, are to be ascribed to lack of geometric similarity of the different sizes of bolts. On the average for all types of threads the ratio of minor to major thread diameter (see fig. 23) increases with increasing bolt diameter; the increase, however, is not uniform. Many of the curves for impact specific work in figure 22, particularly those for $5 / 8$ in. bolts having Dardelet threads, show a rough similarity in change of slope to the changes in slope of the curves for ratio of minor to major thread diameters in

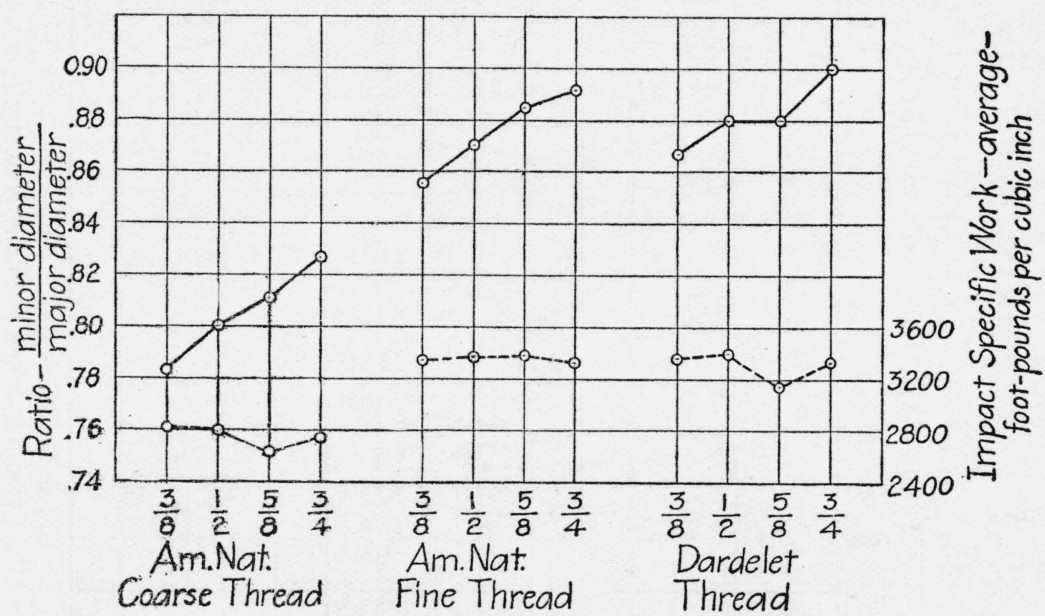

FIGURE 23.-Relation of the major diameter to the ratio between the minor diameter and the major diameter.

figure 23. To eliminate so far as possible the effect of variations in the material the average of the impact specific works for all materials and for each size and type of thread were computed and also plotted in figure 23. The comparison of the curves seems to show a definite relationship between impact specific work and ratio of minor to major thread diameter. Similar but less definite rela tionships are to be noticed in the static specific work, impact effective stretch, and static effective stretch. The variations in the individual values in all cases are too great to allow of a definite numerical evaluation of this effect of lack of similarity. For this reason it was decided to use the average of all sizes of bolts in making further comparisons.

The values in figure 22 were obtained from bolts having one diameter of thread exposed. If the thread had been exposed for a greater distance it is probable that the effective stretch and the specific work would have been greater.

The ratios of the average impact work to the average static work and the ratios of the average impact stretch to the average static stretch for all types of thread and all sizes of bolt are given in table 15 . 
For the brass bolts the ratio of the stretches was greater than the ratio of works indicating that on the average the tensile loads were less under impact than under static loading. For the bronze bolts the ratios were about the same indicating that the tensile loads were about the same. For the steel and the monel metal bolts, the ratios of the works are greater than the ratios of the stretches, indicating that probably the tensile loads were greater under impact than under static loading.

TABLE 15.-Ratios of impact properties to static properties

\begin{tabular}{|c|c|c|c|}
\hline Material & Thread & $\frac{\text { Impact stretch }}{\text { Static stretch }}$ & $\frac{\text { Impact work }}{\text { Static work }}$ \\
\hline Chromium-nickel steel... & $\left\{\begin{array}{l}\text { Coarse } \\
\text { Fine } \\
\text { Dardelet }\end{array}\right.$ & $\begin{array}{l}\text { 1. } 117 \\
\text { 1. } 013 \\
1.312\end{array}$ & $\begin{array}{l}\text { 1. } 190 \\
\text { 1. } 130 \\
\text { 1. } 268\end{array}$ \\
\hline A verage. - & & 1.147 & 1.196 \\
\hline Cold-rolled steel. & $\left\{\begin{array}{l}\text { Coarse } \ldots . . . \\
\text { Fine } \\
\text { Dardelet... }\end{array}\right.$ & $\begin{array}{l}1.207 \\
1.091 \\
1.282 \\
\end{array}$ & $\begin{array}{l}1.348 \\
1.252 \\
1.305 \\
\end{array}$ \\
\hline Average. & & 1.193 & 1.302 \\
\hline Monel metal & $\left\{\begin{array}{l}\text { Coarse } \\
\text { Fine } \\
\text { Dardelet... }\end{array}\right.$ & $\begin{array}{l}1.086 \\
1.083 \\
1.183 \\
\end{array}$ & $\begin{array}{l}1.210 \\
1.215 \\
1.170\end{array}$ \\
\hline Average. & & 1. 117 & 1. 198 \\
\hline Bronze... & $\left\{\begin{array}{l}\text { Coarse } \ldots . . . \\
\text { Fine } \\
\text { Dardelet } \ldots\end{array}\right.$ & $\begin{array}{l}\text { 1. } 270 \\
\text { 1. } 276 \\
\text { 1. } 340\end{array}$ & $\begin{array}{l}1.282 \\
1.250 \\
1.208 \\
\end{array}$ \\
\hline Average... & & 1.295 & 1.247 \\
\hline Brass ... & $\left\{\begin{array}{l}\text { Coarse } \\
\text { Fine } \\
\text { Dardelet }\end{array}\right.$ & $\begin{array}{l}3.104 \\
2.702 \\
2.594 \\
\end{array}$ & $\begin{array}{l}2.790 \\
2.450 \\
2.072\end{array}$ \\
\hline A verage _ - & & 2.800 & 2.437 \\
\hline
\end{tabular}

It seemed desirable to make a more detailed comparison of the relative effects of load and stretch in comparing static work with impact work. For this purpose, for each material, type of thread, and bolt diameter an "average static stress" (and an "average impact stress") was computed by dividing the "static specific work" (or "impact specific work") expressed in inch-pounds by the "static effective stretch" (or "impact effective stretch"). If the stretch used were the stretch under load at the time of fracture the "stresses" so computed would be the average of the tensile stresses during the breaking of the bolt. The actual stretches under load at the time of fracture could not be determined in the impact tests so that instead for both static and impact loads the residual stretches of the fractured specimens were used. The stresses thus computed were therefore larger than the actual average of the stresses during the breaking of the bolt. For comparative purposes, however, they gave a measure of the relative strength of the bolts under static and impact loads.

In addition an average "ultimate tensile strength" under static load of the material at the root of the threads was computed by dividing the maximum static load by the area at the root of the threads. Some consistent differences between the three types of thread were 
found by comparing the results of these computations. For all materials both the average ultimate tensile strength and the average static stress for the American fine thread were smaller than for the other threads, the difference in the average static stress being in all cases greater than in the ultimate tensile strength.

The average impact stress, however, was for all materials greatest for the American coarse thread and (except for the bronze bolts) least for the Dardelet.

The effective stretch showed nearly the reverse of these relationships. For all materials except brass the static effective stretch was greatest for the American fine thread. For all materials the impact effective stretch was least for the American coarse thread and nearly equal for the American fine and Dardelet, averaging slightly higher for the Dardelet.

The consistency of these differences between the different types of thread indicates that for all the materials the type of thread affects in a consistent way each of the factors, static strength, static stretch, impact strength, and impact stretch upon which the ratio of impact work to static work and the ratio of the impact stretch to static stretch depend. The magnitude of the effect upon each factor, however, is different for each material so that similar consistency is not found in the values given in table 15 .

It would be desirable to know definitely how each of these factors is influenced by the details of thread shape, such as depth of notch, angle at root, etc., and how this influence differs for different materials. This would, however, require an extensive further investigation. The present investigation planned primarily to give information about commercial forms of bolts, does not furnish the necessary data.

That in general the impact work is greater than the static work is confirmed by other investigators.

Beyer's ${ }^{10}$ data given in his reports of tests on $1 / 4$ in. and $5 / 16$ in. diameter cold-drawn steel bolts having U. S. Standard threads and Dardelet threads show that the ratio of impact to static work ranges from 0.82 to 2.31 , the average ratio being 1.45 . This wide range of ratios is probably due to the erratic behavior of cold-drawn steel in impact.

Russell ${ }^{11}$ found from transverse tests of rectangular cast-iron bars that the impact work was almost 44 percent greater than the static work. He suggests that this difference may be due to the increase of temperature under impact.

In another study, Russell ${ }^{12}$ tested four tensile impact specimens and two static tensile specimens cut from flat bars of different thicknesses. The reduced section was 1 in. in length and $1 / 4$ in. in width, while the thickness varied. The impact work was greater than the static work as follows: Norway iron, 23.9 percent; Tennessee charcoal iron, 20.9 percent; Tennessee common iron, 39.4 percent; and soft Bessemer tire steel, 29.6 percent.

\footnotetext{
10 Report no. 2162 (Nov. 1929) and no. 2162A (Feb. 1930), Comparative Shock Resistance of Standard V Thread and Nut Connections and Dardelet Thread and Nut Connections. Columbia University (New York, N. Y.). Report no. 2207 (June 1930), Effect of Length of Thread Exposure upon the Static Tensile Strength and Energy to Rupture of Standard V and Dardelet Thread and Nut Connections. Columbia University (New York, N. Y.).

11 Experiments with a new machine for testing materials under impact, Proc. Am. Soc. Civil Engrs. 39, 237 (1898).

12 Tension impact tests of rolled steel. Eng. News 45, (no. 1) 14 (Jan. 3, 1901).
} 
Hatt ${ }^{13}$ found that there was not much difference between the tensile impact work and the tensile static work for most metals but that the impact work was somewhat greater for steel castings.

Blount, Kirkaldy, and Sanky ${ }^{14}$ concluded from their tests "that the energy absorbed per cubic inch in the impact-tensile test is considerably greater than in the static-tensile test, the ratio being approximately 1.6, which may be due to the suddenness of the action".

Cornu-Thenard ${ }^{15}$ in 1919 made transverse tests of specimens 10 by 10 by $53.3 \mathrm{~mm}$ notched in accordance with the recommendations made at the Copenhagen Congress of the International Association for Testing Materials. For the shock tests the speed was $5.30 \mathrm{mps}$ and for the static tests $60 \mathrm{mph}$. His results are as follows:

Ratio of impact to static work

Extra soit _...

Half-hard, nickel, heat treated. 01

Hard, chromium-nickel, heat treated

Hard, chromium-nickel, annealed

Extra soft, coarse grained structure

They show that except for very soft steel the impact work is greater than the static work. The difference for the chromiumnickel steel, heat treated, is about one-half that for our bolt specimens.

McAdam ${ }^{16}$ in discussing the relation of the impact work to the static work refers to the work of other investigators and summarizes the work done on notched specimens at the United States Naval Experiment Station. Carbon and alloy steels were used. The ratio of the impact work to the static work varied from 1.03 to 1.72 , the average being 1.36. He concludes that the available data indicate that the impact work, in general, is greater than the static work, because usually the stretch and reduction of area for impact specimens are greater than for static specimens.

Nisley, ${ }^{17}$ at Watertown Arsenal, investigated the relation between the impact and static tensile works using specimens machined to a diameter of 0.543 in. for a distance of 2 in. The investigation was limited to one steel, a carbon content 0.39 percent, and six different heat treatments. Five specimens of each heat treatment were tested in the same Charpy impact machine as used in our tests of bolts, and five specimens of each heat treatment were tested in static tension.

The average ratio of impact work to static work varied from 1.63 for heat treatment " $\mathrm{A}$ " $\left(6 \mathrm{hr}\right.$ at $1,100^{\circ} \mathrm{C}$ and furnace cooled) to 1.31 for heat treatment " $\mathrm{E}$ " $\left(1 \mathrm{hr}\right.$ at $850^{\circ} \mathrm{C}$ and water quenched, drawn $1 \mathrm{hr}$ at $600^{\circ} \mathrm{C}$ and air cooled).

Petrenko ${ }^{18}$ made transverse tests on notched specimens, using a Humfrey machine for the static tests, and an Izod machine for the impact tests. In each of these machines the specimen is fixed at one end and the force applied at the other end. Four notches having different dimensions were used.

Although Petrenko's numerical values do not agree closely with our results on bolts, as is to be expected because of the differences in

\footnotetext{
13 Tensile impact tests of metals, Proc. Am. Soc. Testing Materials, 4, 281 (1904).

${ }^{14}$ Comparison of the tensile, impact-tensile, and repeated-bending methods of testing steel, Proc. Inst. Mech. Lingrs. 1-2, 715 (1910)

${ }_{15}$ Sur les essais des flexion par choc de barreaux entaillés, Comp. Rend. Acad. d Sci. 168, 1315 (June 30, 1919 ).

${ }_{16}$ Impact tests of metals. Proc. Am. Soc. Testing Materials, 22, part II, 37 (1922).

17 The relation between the dynamic and the static tensile tests, Army Ordnance, 20, 88-93 (Sept., Oct., 1923).

${ }_{18}$ Comparative slow bend and impact notched bar tests on some metals, BS Tech. Pap. 19, (1924-25) T289.
} 
the specimens and the method of applying the loads, the high ratios, particularly for brass, in our tests of bolts are in general agreement with his results.

A very careful and complete investigation of the relation between static work and impact work was made by H. C. Mann at Watertown Arsenal. ${ }^{19}$ The specimens were machined to a diameter of 0.5 in. for a distance of $2.5 \mathrm{in}$. The shoulder fillets had a radius of $0.062 \mathrm{in}$. Three specimens of each material and heat treatment were tested in the Charpy impact machine having a capacity of $2,170 \mathrm{ft}-\mathrm{lb}$, and three of each in static tension. In the static test the stretch up to point of rupture was measured between the shoulders, using a dial micrometer. The chemical compositions of the steels are given in table 16 and the results of the impact and the static tensile tests are given in table 17 .

TABLE 16.-Chemical composition of steels used in impact and static tensile tests from Watertown Arsenal Experimental Report no. 319

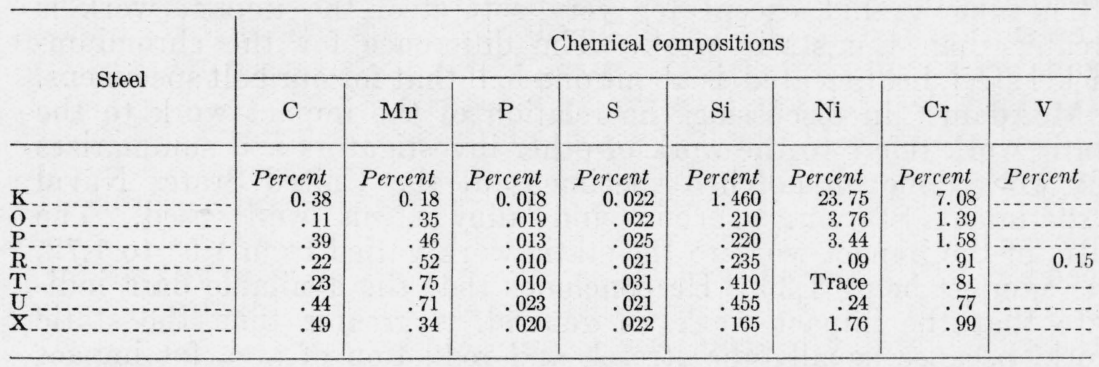

TABLE 17.-Results of impact and static tensile tests from Watertown Arsenal Experimental Report no. 319

\begin{tabular}{|c|c|c|c|c|c|c|}
\hline \multicolumn{2}{|r|}{ Steel } & \multirow{2}{*}{$\begin{array}{l}\text { Microstructure after } \\
\text { heat treatment }\end{array}$} & \multirow{2}{*}{$\begin{array}{l}\text { Brinell } \\
\text { number }\end{array}$} & \multirow{2}{*}{$\begin{array}{l}\text { Impact } \\
\text { work, } \\
\text { average }\end{array}$} & \multirow{2}{*}{$\begin{array}{l}\text { Static } \\
\text { work, } \\
\text { average }\end{array}$} & \multirow{2}{*}{$\begin{array}{c}\text { Ratio } \\
\text { Impact work } \\
\text { Static work }\end{array}$} \\
\hline Symbol & Description & & & & & \\
\hline & & (Austenitic & 207 & $\begin{array}{l}\mathrm{ft}-1 \mathrm{~b} \\
1,250\end{array}$ & $\begin{array}{l}\mathrm{ft}-\mathrm{lb} \\
983\end{array}$ & 1. 27 \\
\hline $\mathbf{K}$ & "Stainless". & & 196 & 1,190 & 933 & 1. 28 \\
\hline 0 & SSAE no. $3,312 \ldots$ & Sorbo-pearlitic & 187 & 790 & 584 & 1.35 \\
\hline $\mathrm{P}$ & SAE no. 3,340 nickel- & Sorbitic & $\begin{array}{l}196 \\
269\end{array}$ & $\begin{array}{l}940 \\
953\end{array}$ & $\begin{array}{l}669 \\
718\end{array}$ & $\begin{array}{l}1.40 \\
1.33\end{array}$ \\
\hline $\mathrm{R}$ & $\begin{array}{l}\text { chromium. } \\
\text { SSAE no. } 6,120\end{array}$ & Sorbo-pearlitic. & 149 & 937 & 631 & 1.48 \\
\hline & Chromium-vanadium .... & _... do & 217 & 924 & 656 & 1. 41 \\
\hline $\mathrm{T}$ & $\left\{\begin{array}{l}\text { SAE no. } 5,120 \\
\text { Case hardening steel }\end{array}\right.$ & Sorbitic & $\begin{array}{l}143 \\
196\end{array}$ & $\begin{array}{r}924 \\
1,023\end{array}$ & $\begin{array}{l}650 \\
743\end{array}$ & 1. 42 \\
\hline 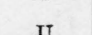 & Coase nardening steet.. & $\{\ldots$ do $\ldots$ & 207 & $\begin{array}{l}1,020 \\
947\end{array}$ & 694 & $\begin{array}{l}\text { 1. } 38 \\
1.36\end{array}$ \\
\hline U & (E) 10.5 & Troosto-Sorbitic & $\begin{array}{l}207 \\
228\end{array}$ & $\begin{array}{r}980 \\
1,003\end{array}$ & $\begin{array}{l}709 \\
750\end{array}$ & $\begin{array}{l}\text { 1. } 38 \\
1.34\end{array}$ \\
\hline $\mathrm{X}$ & SAE no. 3,250 & $\left\{\begin{array}{l}\text { Troosto-Sorbitic } \\
\text { Sorbitic_......... }\end{array}\right.$ & $\begin{array}{l}228 \\
228\end{array}$ & $\begin{array}{r}1,000 \\
993\end{array}$ & 735 & $\begin{array}{l}1.54 \\
1.35\end{array}$ \\
\hline
\end{tabular}

The report states that "The effect of volume has been investigated sufficiently to establish the fact that specimens of different volume but of the same microstructure absorb an amount of the energy of impact in proportion to the volume."

19 Watertown Arsenal Experimental Report no. 319. 


\section{CONCLUSIONS}

Impact and static tensile tests were made on bolts with nuts having American National coarse threads, American National fine threads, and Dardelet threads. The nominal diameters of the bolts were $3 / 8$, $1 / 2,5 / 8$, and $3 / 4$ in. The materials were heat-treated chromium-nickel steel, cold-rolled steel (Bessemer screw stock), monel metal, bronze (coppersilicon-manganese alloy), and brass. The length of each bolt from the head to the bearing face of the nut was five times the nominal diameter. The exposed threads on the bolt extended from the bearing face of the nut toward the head for one diameter. Four similar specimens of each material, diameter, and thread were tested under single-blow impact loading and two under static loading. The total number of specimens was 360 . The following conclusions were drawn from the results of the tests:

1. In the impact tests two chromium-nickel bolts with American National fine threads failed by stripping the thread. Over one-fourth of the cold-rolled steel bolts with American National threads, both coarse and fine, had "brittle" failures. These anomalous failures gave erratic and low impact values. Otherwise, similar bolts having: Dardelet threads gave no such erratic results.

2. Except for the brass bolts and those which showed stripped threads or brittle failures, the work per unit volume required to rupture the bolts either under impact loading or under static loading was for each material and for each type of thread approximately independent of the diameter of the bolts. There was, however, an indication that the work per unit volume was dependent somewhat upon the ratio of minor to major diameter of the thread.

3. Except for the brass bolts and those which had stripped threads or brittle failures, the stretch in the one diameter of exposed threads either under impact loading or under static loading was for each material and each type of thread approximately independent of the diameter of the bolts.

4. In all cases the impact works for bolts having American National coarse threads were less than the impact works for bolts of the same size and material having American National fine threads.

5. Except for the brass bolts and those which showed stripped threads or brittle failures, the impact works for bolts having American National fine threads were approximately the same as the impact works for bolts of the same size and material having Dardelet threads. The impact works for brass bolts having American National fine threads were on the average 12 percent less than the impact works for brass bolts of the same size having Dardelet threads.

6. In all cases the impact works for bolts having Dardelet threads were much greater than the impact works for bolts of the same size and material having American National coarse threads.

7. In all cases the static works for bolts having American National coarse threads were less than the static works for bolts of the same size and material having American National fine threads.

8. Except for the brass bolts and those which showed stripped threads or brittle failures, the static works for bolts having American National fine threads were approximately the same as the static works for bolts of the same size and material having Dardelet threads. For the brass bolts the static works for bolts having American Na- 
tional fine threads were on the average approximately 26 percent lower than the static works for bolts of the same size having Dardelet threads.

9. In all cases the static works for bolts having Dardelet threads were greater than the static works for bolts of the same size and material having American National coarse threads.

10. In all cases the maximum static loads for bolts having American National coarse threads were less than the maximum static loads for bolts of the same size and material having American National fine threads.

11. In all cases the maximum static loads for bolts having American National fine threads were approximately the same as the maximum static loads for bolts of the same size and material having Dardelet threads.

12. In all cases the maximum static loads for bolts having Dardelet threads were greater than the maximum static loads for bolts of the same size and material having American National coarse threads.

13. For bolts of the same size and having the same threads the static-bolt efficiencies, defined as the ratio of the maximum static load for the bolt to the load computed by multiplying the cross-sectional area of the shank by the ultimate tensile strength of the material, were approximately the same for all of the materials.

Washington, December 19, 1934. 